\title{
WestVirginiaUniversity
}

THE RESEARCH REPOSITORY @ WVU

Graduate Theses, Dissertations, and Problem Reports

2014

\section{Synchronization Behavior in Coupled Chemical Oscillators}

\author{
Simbarashe Nkomo
}

Follow this and additional works at: https://researchrepository.wvu.edu/etd

\section{Recommended Citation}

Nkomo, Simbarashe, "Synchronization Behavior in Coupled Chemical Oscillators" (2014). Graduate Theses, Dissertations, and Problem Reports. 7338.

https://researchrepository.wvu.edu/etd/7338

This Dissertation is protected by copyright and/or related rights. It has been brought to you by the The Research Repository @ WVU with permission from the rights-holder(s). You are free to use this Dissertation in any way that is permitted by the copyright and related rights legislation that applies to your use. For other uses you must obtain permission from the rights-holder(s) directly, unless additional rights are indicated by a Creative Commons license in the record and/ or on the work itself. This Dissertation has been accepted for inclusion in WVU Graduate Theses, Dissertations, and Problem Reports collection by an authorized administrator of The Research Repository @ WVU.

For more information, please contact researchrepository@mail.wvu.edu. 


\title{
Synchronization Behavior in Coupled Chemical Oscillators
}

\author{
Simbarashe Nkomo
}

\section{DISSERTATION}

\author{
Submitted to the Eberly College of Arts and Sciences at \\ West Virginia University \\ in Partial Fulfillment of the Requirements for \\ the Degree of \\ Doctor of Philosophy \\ in \\ Chemistry
}
Kenneth Showalter, Ph.D., Chair Charles Jaffé, Ph.D.
Terry Gullion, Ph.D.
Justin Legleiter, Ph.D.
Mark R. Tinsley, Ph.D.
Mikel Holcomb, Ph.D.
Morgantown, West Virginia
2014

Keywords: BZ Reaction, Chimera States, Synchronization, Multistability, Phase Response Curves, Phase-Slip Behavior 
UMI Number: 3618130

All rights reserved

INFORMATION TO ALL USERS

The quality of this reproduction is dependent upon the quality of the copy submitted.

In the unlikely event that the author did not send a complete manuscript and there are missing pages, these will be noted. Also, if material had to be removed, a note will indicate the deletion.

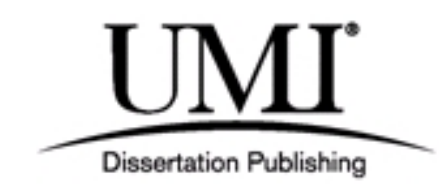

UMI 3618130

Published by ProQuest LLC (2014). Copyright in the Dissertation held by the Author.

Microform Edition () ProQuest LLC.

All rights reserved. This work is protected against unauthorized copying under Title 17, United States Code

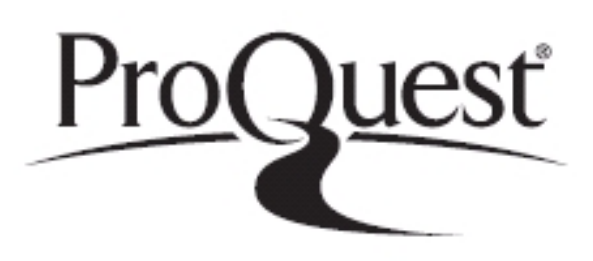

ProQuest LLC.

789 East Eisenhower Parkway

P.O. Box 1346

Ann Arbor, MI 48106 - 1346 


\title{
ABSTRACT
}

\section{Synchronization Behavior in Coupled Chemical Oscillators}

\author{
Simbarashe Nkomo
}

Synchronization is a collective phenomenon emerging from the interactions of different dynamical systems. Systems with different characteristics adjust their behavior to a common behavior of the group. This collective behavior is observed in many biological, chemical, and physical systems. Examples from different fields include pacemaker heart cells, synchronization of neurons during epilepsy seizures, arrays of microwave oscillators, and robot manipulators. Studies of coupled os-

cillators have revealed different mechanisms by which discrete oscillators interact and organize to a uniform synchronized state from an incoherent state. The discovery of a new type of synchronization state, called the chimera state has further broadened the field of synchronization. A chimera state is made up of coexisting subpopulations of oscillators, each with same coupling structure, but with one exhibiting synchronous behavior and the other asynchronous behavior. The phenomena has been the focus of much theoretical and experimental research in the past decade. In this thesis, experimental and simulation studies of chimera states in populations of coupled chemical oscillators will be described and their relation to other synchronization states will be characterized. Experiments were carried out with the photosensitive Belousov-Zhabotinsky (BZ) chemical oscillators and a light feedback scheme. The dimensionless two-variable Zhabotinsky-Buchholtz-Kiyatin-Epstein (ZBKE) model of the BZ chemical system was used in simulations. 
A two-group coupling model, which splits the oscillators into two subpopulations, was used in the first part of the study. The subpopulations are globally coupled, both within and between the subpopulations. The coupling of every oscillator with members of the other subpopulation is weaker than the coupling with members of its own subpopulation. In-phase, out-of-phase, and phase-cluster synchronized states, as well as the chimera state, were found in both experiments and simulations. The probability of finding a chimera state decreases with increasing intra-group coupling strength. The study also revealed that heterogeneity in the frequencies of the oscillators in the system decreases the lifetime of a chimera. This was evidenced by the collapse of the chimera state to a synchronized state in both experiments and simulations with heterogeneous oscillators.

Synchronized and mixed-state behaviors are observed in populations of nonlocally coupled chemical oscillators in a ring configuration. With nonlocal coupling, the nearest neighbors are strongly coupled and the coupling strength decreases exponentially with distance. Experimental studies show stable chimera states, phase cluster states and phase waves coexisting with unsychronized groups of oscillators. These are spontaneously formed from quasi-random initial phase distributions in the experiments and random initial phase distributions in simulations. Simulations with homogeneous and heterogeneous oscillators revealed that a finite spread of frequencies increases the probability of initiating a synchronized group, leading to chimera states. The effects of group size and coupling strength on chimera states, phase waves, phase clusters, and traveling waves are discussed. Complex behaviors in coexisting states were analyzed, consisting of periodic phase slips with identical oscillators and periodic switching with nonidentical oscillators. Fourier transform analysis was used to distinguish between states exhibiting high periodicity and chimera states, which show similar average behavior. 


\section{Acknowledgements}

I sincerely appreciate my advisor, Dr. Kenneth Showalter for providing a great research atmosphere to pursue my studies and opportunities for professional development. Without his guidance, all the research would not have been possible. I also want to express my gratitude to all the committee members: Dr. Terry Gullion, Dr. Justin Legleiter, Dr. Martin Ferer, Dr. Mikel Holcomb, and Dr. Charles Jaffé for taking time to evaluate my dissertation. My special thanks go to Dr. Mark Tinsley for his research insights and help with experiments. Thank you for the time spent answering questions and for providing valuable suggestions for my dissertation. I also want to express my gratitude to the research group members: Dr. Hua Ke, Dr. Fang Wang, Dr. Calistus Ngonhala, Desmond Yengi, Darrell Collison, and Razan Snari, for their help during my graduate education. I would like to thank my family and friends for the encouragement and support throughout my education. 


\section{Contents}

$\begin{array}{ll}\text { Abstract } & \text { i }\end{array}$

$\begin{array}{ll}\text { Acknowledgements } & \text { iii }\end{array}$

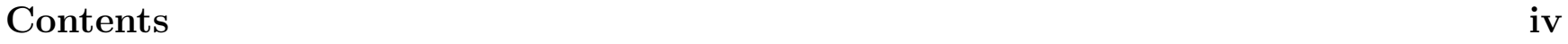

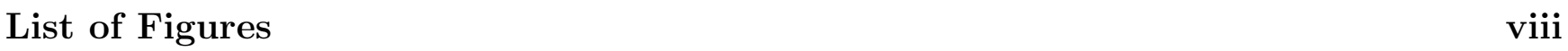

$\begin{array}{lc}\text { List of Tables } & \text { xi }\end{array}$

1 Synchronization $\quad 1$

1.1 Background . . . . . . . . . . . . . . . . . . . . 2

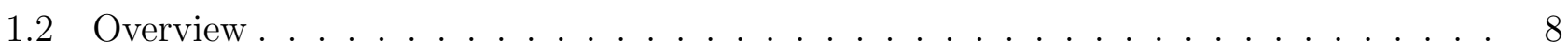

2 The Belousov-Zhabotinsky Chemistry and Models 16

2.1 Introduction . . . . . . . . . . . . . . . . . . 16

2.2 The Belousov-Zhabotinsky Reaction . . . . . . . . . . . . . . . 16

2.2.1 The FKN Mechanism . . . . . . . . . . . . . . . . . . . . . 17

2.2.2 The ZBKE Reaction Scheme and Model . . . . . . . . . . . . . . 18

2.2.3 Photosensitive BZ Reaction System . . . . . . . . . . . . . . . . 22

2.2.4 The Modified ZBKE Model . . . . . . . . . . . . . . . . . . . . . . . 23 
2.3 Experiment Setup . . . . . . . . . . . . . . . . . . . . . . . . . . . 25

2.3 .1 Instrumentation . . . . . . . . . . . . . . . . . . . . . 25

2.3.2 Preparation of Ruthenium(ll) Catalyst-Loaded Beads . . . . . . . . . . . . 26

2.3.3 Preparation of Catalyst-Free BZ Solution . . . . . . . . . . . . . . . 26

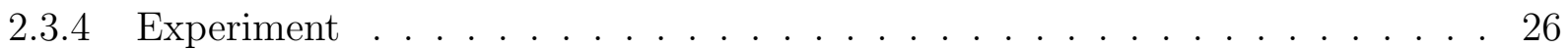

2.4 Phase Response Curves . . . . . . . . . . . . . . . . . . . . . . . . . . . . . . . . . 28

2.4 .1 Introduction . . . . . . . . . . . . . . . . . . 28

2.4 .2 Phase Response Experiments . . . . . . . . . . . . . . . . . . . . . . . . . . 29

2.4 .3 Results . . . . . . . . . . . . . . . . . . . . . 30

3 Chimera and Phase-cluster States 40

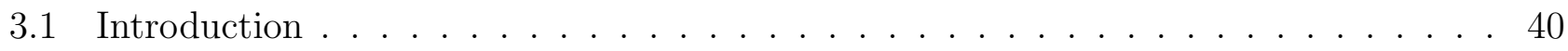

3.2 Coupling Model Background . . . . . . . . . . . . . . . . . . . . . . . . . . . . 41

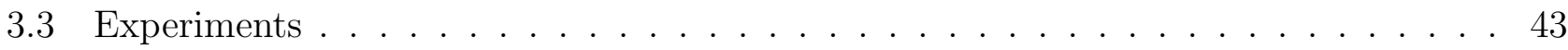

3.4 Data Analysis . . . . . . . . . . . . . . . . . . . . . . . . . . 44

3.4 .1 Mean Signal . . . . . . . . . . . . . . . . . . . . . . . . . . . 44

3.4 .2 Order Parameter . . . . . . . . . . . . . . . . . . . . 44 44

3.4.3 Period and Phase Determination _. . . . . . . . . . . . . 47

3.5 Experiment Results . . . . . . . . . . . . . . . . . . . . . . 47

3.6 Simulations . . . . . . . . . . . . . . . . . . . . . . . 49

3.6 .1 Model . . . . . . . . . . . . . . . . . . . . . . . . . . . . . . . 49

3.6 .2 Simulation Results . . . . . . . . . . . . . . . . . . . 51

3.7 Multistability Experiments . . . . . . . . . . . . . . . . . . . 55

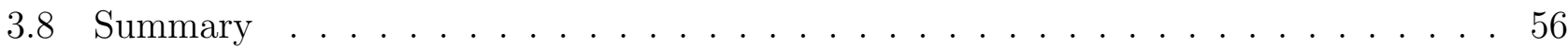


4.1 Introduction . . . . . . . . . . . . . . . . . . . . 66

4.2 Nonlocal Coupling Scheme . . . . . . . . . . . . . . . . . 67

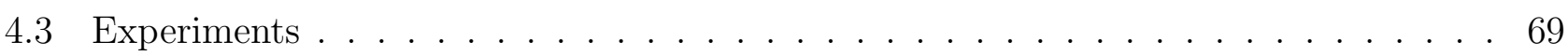

4.3.1 Chimera State from Quasi-Random Initial Phase Distribution . . . . . . . . 70

4.3.2 Chimera State from Special Initial Conditions . . . . . . . . . . . . 71

4.3 .3 Synchronized States . . . . . . . . . . . . . . . . 71

4.3.4 Phase-Cluster Chimera States . . . . . . . . . . . . . . . . 74

4.3.5 Phase-Wave Chimera States . . . . . . . . . . . . . . . . . 74

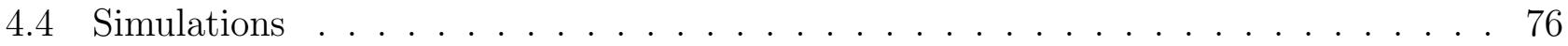

4.4.1 Chimera State . . . . . . . . . . . . . . . . 77

4.4.2 Phase-Cluster Chimera States . . . . . . . . . . . . . . . . . . 78

4.4 .3 Phase-Wave Chimera States . . . . . . . . . . . . . . . . . . . 80

4.5 Spiral Chimera States . . . . . . . . . . . . . . . . . . 82

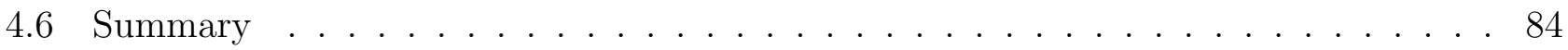

5 Analysis of Dynamical Regimes in Coupled Chemical Oscillators 88

5.1 Introduction . . . . . . . . . . . . . . . . . . 88

5.2 Varying $\kappa \ldots \ldots \ldots \ldots . \ldots \ldots . \ldots \ldots$

5.2.1 Summary of Experiment Results . . . . . . . . . . . . . . . . 89

5.3 Simulation Results . . . . . . . . . . . . . . . . . . . . . 91

5.3 .1 Varying $\kappa \ldots \ldots \ldots \ldots \ldots \ldots$

5.3 .2 Varying $\mathrm{N} \ldots \ldots \ldots \ldots$

5.4 Analysis of Experimental and Numerical Results . . . . . . . . . . . . . . . . . 95 
$5.4 .1 \quad$ Phase Dispersed State . . . . . . . . . . . . . . . . . . . . 95

5.4 .2 Synchronized States _ . . . . . . . . . . . . . . . . . . . . 95

5.4 .3 Chimera States . . . . . . . . . . . . . . . . . . . . . . . . 97

5.4.3.1 Chimera States in Experiments . . . . . . . . . . . . . . 97

5.4.3.2 Chimera States in Homogeneous Chemical Oscillators . . . . . . . 98

5.4.3.3 Chimera States in Heterogeneous Chemical Oscillators . . . . . . 102

5.4.3.4 Phase-Cluster Chimera States . . . . . . . . . . . . . . . . . . . 104

5.4 .4 Periodic States f . . . . . . . . . . . . . . . . . 107

5.4.4.1 Phase Slip . . . . . . . . . . . . . . . . . . . . 107

5.4.4.2 Switching Behavior . . . . . . . . . . . . . . . . . . 108

5.4.5 Drifting Phase-Cluster Chimera State (DPCS) . . . . . . . . . . . . . 111

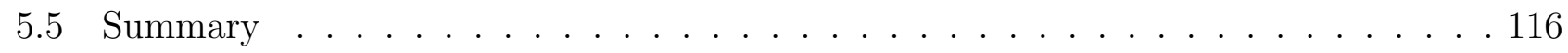




\section{List of Figures}

1.1 Phase potrait for a system of equations describing a violin string . . . . . . . . . . 3

1.2 Geometrical representation of the order parameter . . . . . . . . . . . . . . 4

1.3 Kuramoto model simulations . . . . . . . . . . . . . . . . . . . . . . . 6

1.4 Power spectrum of sinusoidal and relaxation oscillations . . . . . . . . . . . . . . . 8

2.1 (a) Time series of the activator species $x$ and (b) time series of the oxidized form of catalyst. . . . . . . . . . . . . . . . . . . . 24

2.2 Experiment set-up . . . . . . . . . . . . . . . . . . . . . . . 25

2.3 Experiment time series . . . . . . . . . . . . . . . . . . . . 28

2.4 Experiment time series showing (a) phase advancement $\left(\mathrm{T}>\mathrm{T}^{\prime}\right)$ and (b) phase retardation $\left(\mathrm{T}<\mathrm{T}^{\prime}\right) \ldots \ldots \ldots \ldots \ldots \ldots \ldots \ldots \ldots$

2.5 Phase response curves responding to different light perturbations . . . . . . . . . . . 32

2.6 Error margin associated with each point of the phase response curve. . . . . . . . . 33

3.1 Simplified representation of the two-group coupling model . . . . . . . . . . . . 42

3.2 Experiments showing variations of individual intensities of (a) asynchronous oscillators, (b) synchronizing oscillators, and the mean signal with time. . . . . . . . 45

3.3 Normalized mean intensity and the global order order parameter. . . . . . . . . . 46 
3.4 Normalized mean intensity of group A (blue) and group B (red) as a function of time, and (inset) snapshot of the phases of oscillators . . . . . . . . . . . 48

3.5 Dependence of the chimera and cluster states on parameters. . . . . . . . . . . 50

3.6 Long-lived and transient chimera states in simulations and experiments. . . . . . . . 54

3.7 Continuity results showing stable regions for the 1-1, 1-c and 1-n states vs time delay $(\tau) \ldots \ldots \ldots \ldots \ldots \ldots \ldots \ldots$

3.8 Experiment showing stable 1-3 culster state before perturbation and 1-1 state after

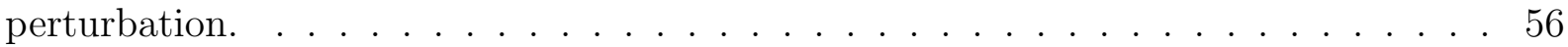

4.1 Coupling strength vs $i^{\text {th }}$ neighbor for different values of $\kappa \ldots . . . . . . . . .668$

4.2 Nonlocal coupling illustrated for oscillator indexed $j=1$ with a coupling radius of

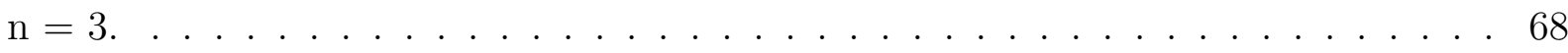

4.3 Experimental behavior of 40 oscillators coupled according to Eq. (4.2) in a ring configuration, with $n=10, \kappa=0.5, K^{\prime}=1, \tau=30$ s. . . . . . . . . 72

4.4 Experimental behavior of 40 oscillators. The experiment was started with a special initial phase distribution of the oscillators. . . . . . . . . . . . . . 73

4.5 Synchronized experimental behavior of 40 oscillators in a ring configuration. . . . . 75

4.6 Measurements showing experimental phase-cluster chimera in 40 coupled BZ oscil-

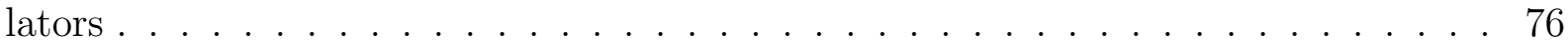

4.7 Experimental phase-wave chimera behavior. . . . . . . . . . . . . . . . . . . 77

4.8 Model simulations of 40 coupled heterogeneous BZ oscillators in a ring configuration. 79

4.9 Phase-cluster chimera states in simulations of 40 coupled heterogeneous BZ oscillators in a ring configuration . . . . . . . . . . . . . . . 80

4.10 Simulations of phase-wave chimera behavior. . . . . . . . . . . . . . . . . . 81

4.11 Simulations of spiral chimera states in populations of BZ oscillators. . . . . . . . . 83 
5.1 Summary of dynamical states observed in experiments. . . . . . . . . . . . . . 90

5.2 The likelihood of finding a chimera-like states as a function of coupling strength . . 92

5.3 Phase dispersed state $(\mathrm{PD}) \ldots \ldots \ldots \ldots$. . . . . . . . . . . . . . 96

5.4 Phase-cluster state from model simulation of 40 homogeneous BZ oscillators. . . . . 97

5.5 Experiment showing a chimera state with BZ chemical oscillators . . . . . . . . . . 99

5.6 Model simulation with 90 homogeneous BZ oscillators. . . . . . . . . . . . . 101

5.7 Power spectra of three oscillators; (a) $j=10$ from the phase dispersed group (green), (b) $j=58$ from the asynchronous group (red), and (c) $j=75$ from the synchronized group (blue). . . . . . . . . . . . . . . . . . . . 102

5.8 Model simulation with 90 heterogeneous BZ oscillators . . . . . . . . . . . . 103

5.9 Power spectra of the Fourier transforms of the time series. . . . . . . . . . . 105

5.10 Phase-cluster chimera state observed in model simulations of 40 coupled heterogeneous BZ oscillators in a ring configuration. . . . . . . . . . . . 106

5.11 Phase slipping behavior in simulation with 80 homogeneous BZ oscillators . . . . . 109 5.12 Switching behavior in 40 heterogeneous BZ oscillators . . . . . . . . . . . . 110

5.13 Switching behavior between phase wave and synchronized oscillators . . . . . . . . 112

5.14 Distribution of phase-cluster chimera states from 200 realizations. . . . . . . . 113

5.15 Model simulations of drifting phase-cluster chimera states . . . . . . . . . . . . . 114

5.16 Linear velocity as a function of group size N. . . . . . . . . . . . . 116 


\section{List of Tables}

2.1 The ZBKE Reaction Scheme $[2] \ldots \ldots \ldots \ldots$

2.2 The ZBKE Model Scalings $[2,6] \ldots \ldots \ldots \ldots \ldots \ldots \ldots \ldots$

2.3 The ZBKE Model Parameter Values $[11-14] \ldots \ldots \ldots \ldots \ldots \ldots$

5.1 Summary of the different observed behaviors . . . . . . . . . . . . . . . 94 


\section{Chapter 1}

\section{Synchronization}

Synchronization was first reported in 1665 by the Dutch physicist Christian Huygens [1]. He observed two pendula that were initially swinging at different frequencies adjust to a common frequency $[1,2]$. Synchronization occurs when different dynamical systems interact and adjust to a common behavior [2]. The phenomenon has been observed in nature in systems such as heart cells [3], insulin secreting cells of the pancreas $[4,5]$ and the synchronous firing of neurons during communication processes in the brain $[2,6,7]$. Groups of flashing fire flies and crickets have been observed to flash $[8,9]$ and chirp [10], respectively, in synchrony $[2,7]$. The concept of synchronization has been applied to other fields, such as engineering arrays of power generators [11] and robot manipulators [12] to enhance performance or increase power output $[2,7]$. In many biological systems, synchronization is the key to maintaining the rhythm of a system, as in mammalian intestines, rhythmic heart beating, and timely hormonal release in the body $[2,7]$.

Theoretical and experimental studies of populations of coupled oscillators have aided our understanding of synchronization phenomena. Most studies have used populations of identical oscillators, which have the same frequency but may have a distribution of phases. Heterogeneous (nonidentical) oscillators have distributions in both their phases and frequencies. The form of the 
distribution of frequencies affects the onset of synchronization. Other factors such as population density [13], magnitude and nature of interactions [14] have been shown to affect the onset of synchronized behavior.

\subsection{Background}

Mathematical studies of synchronization date back to Weiner's work on the generation of rhythms in the brain $[15,16]$. In 1967, Winfree formulated a model of weakly coupled limit-cycle oscillators [17]. In modeling of physical or biological systems, a limit cycle is an isolated periodic solution. Figure 1.1 shows solutions to a system of equations, derived by Rayleigh, describing oscillations of a violin string $[18,19]$. The limit cycle is represented by a closed curve in a phase plot. A limit cycle can be stable, unstable or semistable. It is stable if nearby trajectories are attracted to the limit cycle. Figure 1.1a shows a stable limit cycle, where the trajectories (green and black) in the vector field are attracted to the limit cycle. Figure 1.1b illustrates the decay (black) or growth (green) of the amplitude of the oscillations as they approach the limit cycle. An unstable limit cycle repels nearby trajectories on both sides, while a half-stable limit cycle attracts on one side and repels on the other side of the limit cycle $[19,20]$. Once an oscillator is on a limit cycle, the amplitude remains constant unless a perturbation is applied to the system. A system with a stable limit cycle is capable of self-sustained oscillatory behavior.

With weak coupling of oscillators, variations in amplitude are typically negligible because oscillators relax rapidly to the limit cycle [17]. Therefore, the dynamical behavior of the oscillators can be described by the evolution of their phases [17]. If the coupling is strong, the oscillators are moved away from the limit cycle, resulting in changes in amplitude. Other types of behavior such as oscillator death may occur. By assuming weak coupling, Winfree simplified the system to a 

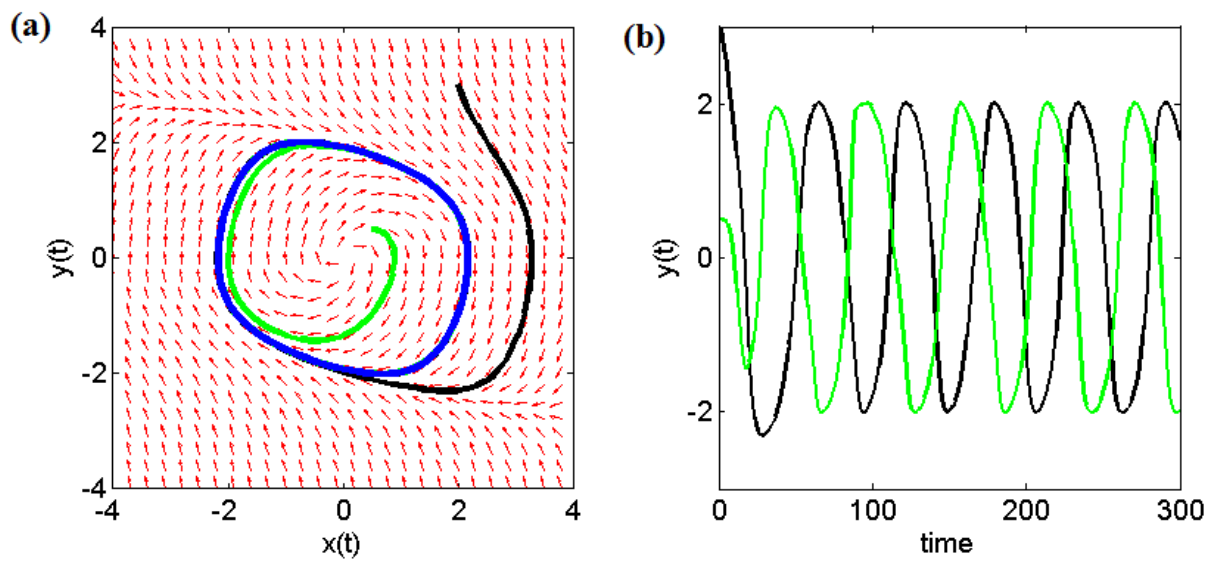

Figure 1.1. (a) Phase potrait for a system of equations describing a violin string [18, 19]. The figure illustrates a stable limit cycle (blue), nearby trajectories (black and green), and the vector field (red arrows). (b) The changes in amplitude of the nearby trajectories (black and green) as they are attracted to the limit cycle.

phase model $[16,17]$. In the model, each oscillator interacts with the mean phase of the population $[16,17]$. In simulations of a system of oscillators with a distribution of intrinsic frequencies, Winfree observed synchronization of oscillators above a certain threshold of coupling [16, 17]. However, the model was not analytically tractable [16]. Kuramoto further developed the idea to a widely studied model of globally coupled phase oscillators with sinusodal coupling [21]. In the Kuramoto model, the dynamical behavior is governed by the following equation:

$$
\dot{\theta}_{i}=\omega_{i}+\sum_{j=1}^{N} K_{i j} \sin \left(\theta_{i}-\theta_{j}\right)
$$

where $\theta_{i}$ and $\omega_{i}$ are the phase and the natural frequency of the $i^{t h}$ oscillator, respectively [21]. The natural frequencies are chosen from a unimodal distribution and $K_{i j}$ is the adjacency matrix $[21,22]$. For uniform coupling, the equation is modified to

$$
\dot{\theta}_{i}=\omega_{i}+\frac{K}{N} \sum_{j=1}^{N} \sin \left(\theta_{i}-\theta_{j}\right)
$$




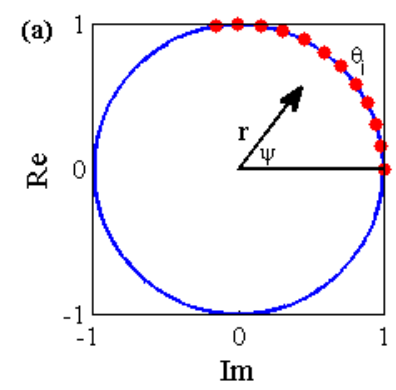

(b)

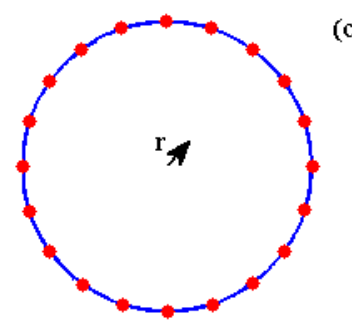

(c)

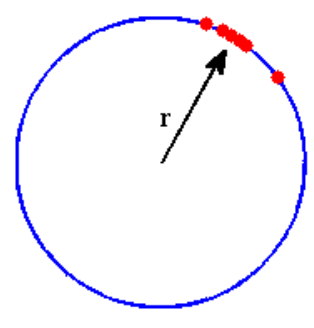

Figure 1.2. (a) Geometrical representation of the order parameter. The figure shows the oscillator phases (red dots); a measure of synchrony is given by the radius $r$, the complex number $r e^{i \psi}$ (arrow) gives the centroid of the phases $\left(\phi_{j}\right)$; and $\psi$ is the average of the phases. (b) An incoherent state with scattered phases has $r \approx 0$. (c) For a coherent state with synchronized phases, the order parameter $r \approx 1$. [16, 21, 22]

where $N$ is the total number of the oscillators [21-23]. The order parameter $r$ was introduced to measure phase coherence of the oscillators. The parameter is given by

$$
r e^{i \psi}=\frac{1}{N} \sum_{j=1}^{N} e^{i \theta_{j}}
$$

where $\psi(t)$ is the average phase and $r(t)$ is a measure of global phase coherence $[16,21,22]$. From the expression

$$
r e^{i \psi}=r(\cos \psi+i \sin \psi)
$$

the order parameter can be represented geometrically, as shown in Figure 1.2a. For an incoherent population of oscillators with phases scattered around the phase circle, $r(t) \approx 0$ (Figure $1.2 \mathrm{~b}$ ), and $r(t) \approx 1$ for in-phase synchronized oscillators, illustrated in Figure 1.2c. 
Expressing the model in terms of the order parameter gives equation $1.5[16,21,22]$

$$
\dot{\theta}_{i}=\omega_{i}+K r \sin \left(\psi-\theta_{i}\right), \quad i=1, \ldots, N
$$

Equation 1.5 gives the interaction of each oscillator with the others through the mean phase $\psi(t)$, i.e., global coupling, and the proportionality of the coupling strength to phase coherence [16]. Model simulations of a unimodal population of oscillators are shown in Figure 1.3. Figure 1.3a shows the initial distribution of frequencies of 200 oscillators chosen randomly from a Gaussian distribution. Synchronizability of such heterogeneous populations of oscillators depends on the width of the distribution. Studies have revealed that a narrower frequency distribution tends to synchronize at lower coupling strength compared to a broader distribution under the same conditions. Complete synchronization in heterogeneous populations of oscillators involves frequency entrainment as well as phase locking $[2,7]$. For a broader distribution, some slower and faster oscillators may not be entrained due to large frequency differences that are outside the entrainment range $[2,7,24]$ or the requirement of a higher value of $K$. In such cases, partially synchronized states as in Figure 1.3b are observed. The oscillators in the middle of the distribution are synchronized, leaving the slower $(\omega<0)$ and faster $(\omega>0)$ oscillators unsynchronized. With a higher value of $K$, the oscillators become frequency locked, as shown in Figure 1.3c. The frequency of the synchronized oscillators depends on the initial frequency distribution. In Figure 1.3c, the synchronized frequency is lower than the distribution average frequency, $\omega_{0}=0$. This is due to asymmetry in the initial frequency distribution of the chosen frequencies.

Figure $1.3 \mathrm{~d}$, shows a threshhold, $K_{c} \approx 1.6$, above which synchronization occurs. Below the critical coupling strength $K_{c}$, there is an incoherent state where oscillators have randomly distributed phases on the phase circle and run at their intrinsic frequencies. For $1.6 \leq K \leq 5$, partial synchronization is observed, where only a fraction of the oscillators are synchronized. In this sim- 

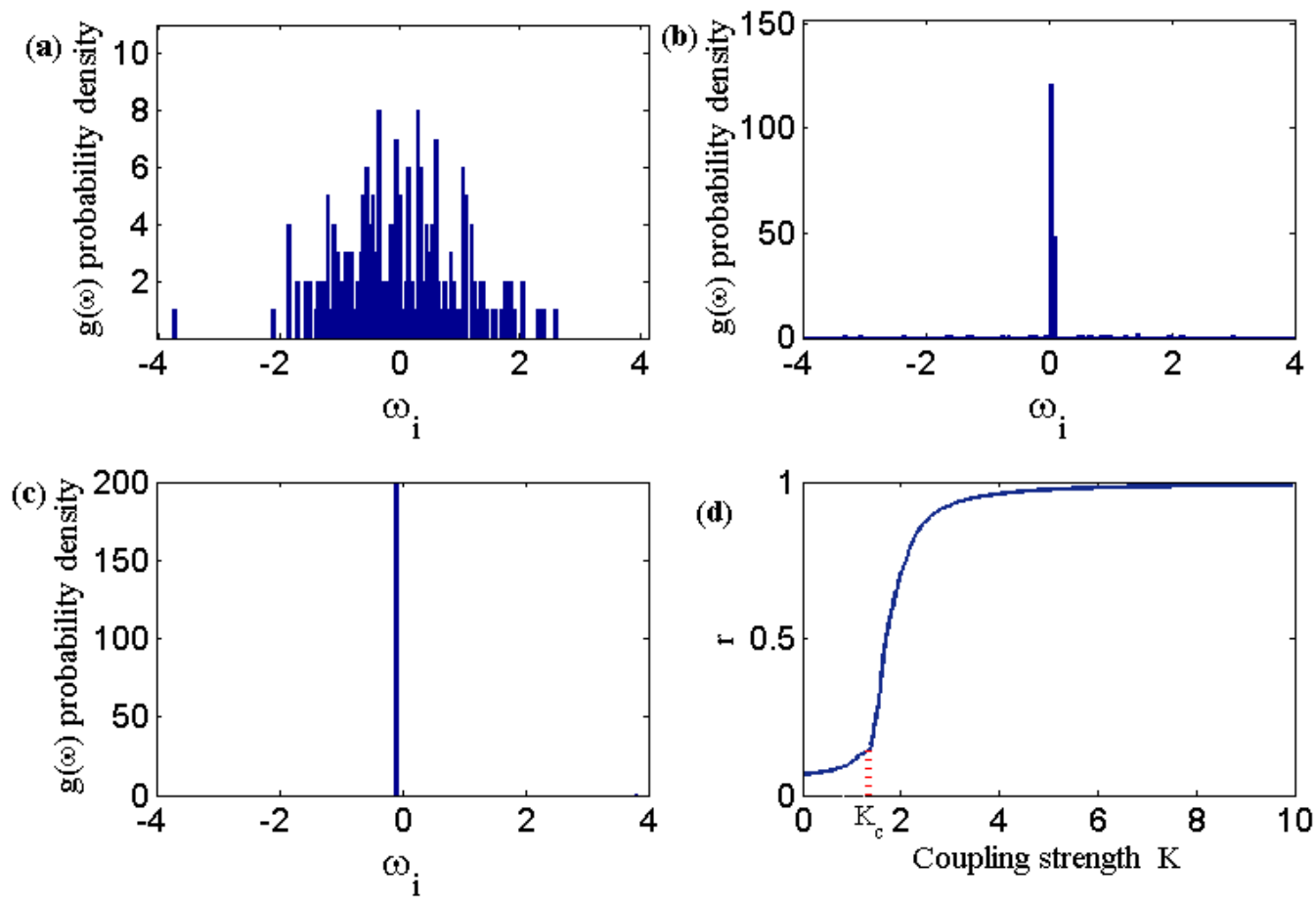

Figure 1.3. (a) Initial frequency distribution of oscillators randomly selected from a normal probability distribution, with $\sigma=1$ and centered about zero for convinience. (b) Partial synchronization of oscillators for $K=3$. (c) Frequency locked oscillators for $K=8$. (d) Phase coherence $r$ as a function of the coupling strength. 
ulation, complete synchronization was observed for values of $K>5$. The value of $K_{c}$ depends on the heterogeneity of the group. Kuramoto analytically derived an expression for $K_{c}$ in terms of the natural frequency distribution $[16,21,22,25]$. For example, for a normal distribution with mean $\mu=0$ and standard deviation $\sigma=1$,

$$
K_{c}=\frac{2}{\pi g(0)}
$$

where

$$
g(0)=\frac{1}{\sigma \sqrt{2 \pi}}
$$

Applying the equations to the distribution in Figure $1.3 \mathrm{a}, \mathrm{g}(0)=0.3989$ and $K_{c}=1.5958$. The analytical value is very close to the value of $K_{c}$ in Figure 1.3d. Expressions have also been derived for other distributions such as the Lorentzian distribution of natural frequencies [22, 25].

Oscillators can also be coupled locally or by other intermediate forms of coupling such as nonlocal coupling. Unlike in global coupling, where each oscillator is coupled to every other oscillator through a common mean field, in local coupling interactions are limited to nearest neighbors. This type of coupling occurs, for example, in mammalian intestines [7]. Neurons in the brain show nonlocal coupling, since each neuron is connected to other neurons that are not necessarily nearest neighbors.

Modified Kuramoto models have been used to study the effects of noise [26], time delay coupling [25, 27-30], and varying network topologies [31]. Multistability and phase clustering behavior, among other behaviors, have been observed in systems with time-delay coupling [25, 27-30]. Applications have extended to systems made up of natural and synthetic oscillators, which exhibit behavior different from sinusoidal oscillations. For example, neurons, heart pacemakers, and the Belousov-Zhabotinsky (BZ) chemical system exhibit relaxation oscillations. These have certain stages of the oscillatory cycle proceeding faster than the others [32, 33]. This results in more than 

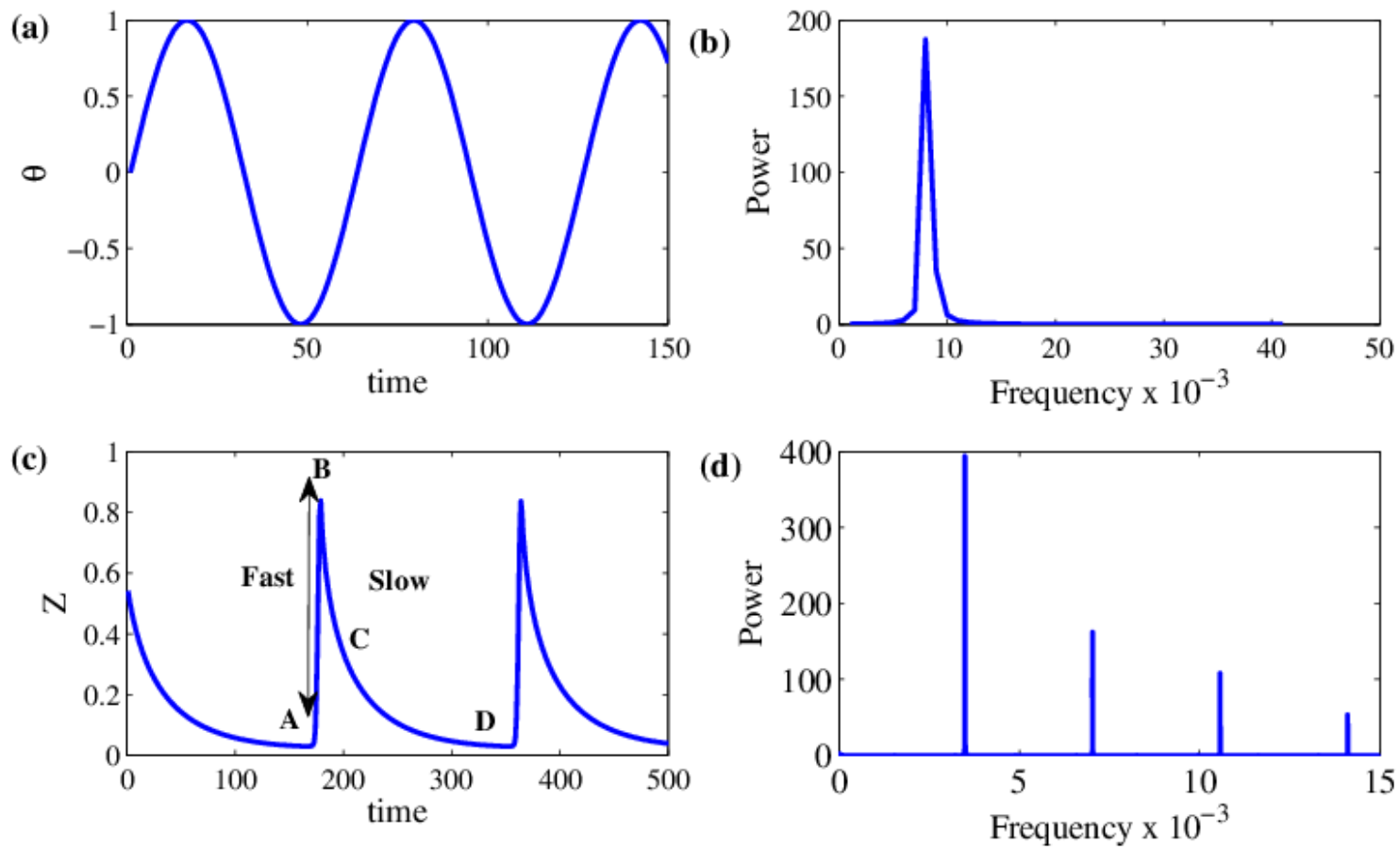

Figure 1.4. (a) Sinusoidal oscillations. (b) Power spectrum showing a single frequency. (c) An example of relaxation oscillations from BZ model simulations. (d) The power spectrum of the relaxation oscillations showing higher frequencies.

one time scale, as illustrated in Figure 1.4. The sinusoidal oscillations (Figure 1.4a) have a single time scale, and the corresponding power spectrum (Figure 1.4b) shows a single peak. However, the oscillator in Figure 1.4c exhibits relaxation oscillations. The change from A to B is very fast, and the system slowly relaxes from B to C to D. The power spectrum now shows higher harmonics, which differentiates these oscillations from the simple harmonic oscillations.

\subsection{Overview}

Studies described in this thesis utilize populations of discrete chemical oscillators, with different coupling structures, to study synchronization behavior. This study is focused mainly on 
the chimera state, which is characterized by coexisiting subpopulations of oscillators exhibiting synchronous and asynchronous behavior. The chemical oscillators are based on the oscillating Belousov-Zhabotinsky (BZ) reaction; the detailed chemistry of the BZ reaction is discussed in Chapter 2. The BZ reaction and its related mathematical models have been used extensively to model biological phenomena [34]. Examples include dynamical quorum sensing in bacterial colonies $[13,35]$, spiral waves linked to ventricular fibrillation [36], hebbian learning in neuroscience [37], synchronization phenomena [38] and waves in subexcitable media [39].

Chapter 3 discusses chimera states and phase cluster states in a simplified two-group coupling model using a predefined phase distribution. The study is extended to a ring of nonlocally coupled oscillators with predefined and random initial phase distributions in Chapter 4. Both experiments and numeric studies of populations of heterogeneous oscillators are discussed. Chapter 5 details the analysis of various dynamical states including simulations of a system of identical chemical oscillators. The experimental signatures of chimera states in the chemical system are also discussed. 


\section{References}

[1] E. Klarreich, "Discovery of coupled oscillation put 17th century scientist ahead of his time." SIAM News 35, 8-1-3 (2002).

Online Version 1

[2] G. Osipov, J. Kurths, and C. Zhou, Synchroization in Oscillatory Networks (Springer, Berlin, 2010). 1,5

[3] C. Peskin, "Mathematical aspects of heart physiology," Courant Institute of Mathematical Science Publication 268-278 (1975). 1

[4] A. Sherman, J. Rinzel, and J. Keizer, "Emergence of organized bursting in clusters of pancreatic beta-cells by channel sharing." Biophys. J. 54, 411-425 (1988).

Online Version 1

[5] A. Sherman and J. Rinzel, "Model for synchronization of pancreatic beta-cells by gap junction coupling." Biophys. J. 59, 547-559 (1991).

Online Version 1

[6] F. Hoppensteadt and E. Izhikevich, Weakly Connected Neural Networks, (Springer-Verlag, New York, 1997). 1

[7] A. Pikovsky, M. Rosenblum, and J. Kurths, Synchronization: A Universal Concept in Nonlinear Sciences (Cambridge University Press, Cambridge, 2003). 1, 5, 7

[8] J. Buck, "Synchronous rhythmic flashing of fireflies. II." Q. Rev. Biol. 63, 265-289 (1988). Online Version 1 
[9] J. Buck and E. Buck, "Mechanism of rhythmic synchronous flashing of fireflies. Fireflies of Southeast Asia may use anticipatory time-measuring in synchronizing their flashing." Science 159, 1319-1327 (1968).

Online Version 1

[10] T. Walker., "Acoustic synchrony: Two mechanisms in the snowy tree cricket." Science $\mathbf{1 6 6}$, 891-894 (1969). 1

[11] Y. Lei, Z. Jiao, M. Wu, and G. Wilde, "Ordered arrays of nanostructures and applications in high-efficient nano-generators," Adv. Eng. Mater. 9, 343-348 (2007).

Online Version 1

[12] H. Nijmeijer and A. Rodriguez-Angeles, "Synchronization of mechanical systems," Int. J. Control 74, 13111323 (2001). 1

[13] A. F. Taylor, M. R. Tinsley, F. Wang, Z. Huang, and K. Showalter, "Dynamical quorum sensing and synchronization in large populations of chemical oscillators." Science 323, 614617 (2009).

Online Version 2, 9

[14] M. Brede, "Local vs global synchronization in networks of non-identical Kuramoto oscillators." Eur. Phys. J. B 62, 87-94 (2008).

Online Version 2

[15] N. Weiner, Nonlinear problems in random theory (MIT Press, Cambridge, 1958). 2

[16] S. H. Strogatz, "From Kuramoto to Crawford: Exploring the onset of synchronization in populations of coupled oscillators," Physica D 143, 1-20 (2000).

Online Version 2, 3, 4, 5, 7 
[17] A. Winfree, "Biological rhythms and the behavior of populations of coupled oscillators." J. Theor. Biol. 16, 15-42 (1967).

Online Version 2, 3

[18] J. Rayleigh, The theory of sound (Dover, New York, 1945). 2, 3

[19] S. Lynch, Dynamical systems with applications using MATLAB (Birkhauser, Boston, 2004). 2, 3

[20] S. H. Strogatz, Nonlinear dynamics and chaos. (Westview, Cambridge, 2000). 2

[21] Y. Kuramoto, Chemical oscillations, waves, and turbulence (Springer-Verlag, New York, 1984).

Online Version 3, 4, 5, 7

[22] K. Ziya, "A review on synchronization through the Kuramoto model," Consortium of the Americas for Interdisciplinary Science (2007). 3, 4, 5, 7

[23] H. Sakaguchi and Y. Kuramoto, "A soluble active rotater model showing phase transitions via mutual entertainment," Prog. Theor. Phys. 76, 576-581 (1986).

Online Version 4

[24] G. C. Sethia, A. Sen, and F. M. Atay, "Clustered chimera states in delay-coupled oscillator systems." Phys. Rev. Lett. 100, 144102 (2008).

Online Version 5

[25] J. A. Acebrón, L. L. Bonilla, C. J. Pérez Vicente, F. Ritort, and R. Spigler, "The Kuramoto model: A simple paradigm for synchronization phenomena," Rev. Mod. Phys. 77, 137-185 
(2005).

Online Version 7

[26] B. C. Bag, K. G. Petrosyan, and C. Hu, "Influence of noise on the synchronization of the stochastic Kuramoto model." Phys. Rev. E 76, 056210 (2007).

Online Version 7

[27] D. V. R. Reddy, A. Sen, and G. L. Johnston, "Experimental evidence of time delay induced death in coupled limit cycle oscillators," Phys. Rev. Lett. 85, 3381-4 (2000).

Online Version 7

[28] M. Choi, H. Kim, D. Kim, and H. Hong, "Synchronization in a system of globally coupled oscillators with time delay," Phys. Rev. E 61, 371-81 (2000).

Online Version

[29] M. K. S. Yeung and S. H. Strogatz, "Time delay in the Kuramoto model of coupled oscillators," Phys. Rev. Lett. 82, 648-651 (1999).

Online Version

[30] M. K. Sen, B. C. Bag, K. G. Petrosyan, and C. Hu, "Effect of time delay on the onset of synchronization of the stochastic Kuramoto model," J. Stat. Mech 2010, P08018 (2010).

Online Version 7

[31] J. G. Restrepo, E. Ott, and B. R. Hunt, "Synchronization in large directed networks of coupled phase oscillators." Chaos 16, 015107 (2006).

Online Version 7

[32] B. van der Pol, "On relaxation oscillations," Philos. Magazine 2, 946-954 (1926). Online Version 7 
[33] S. R. Campbell and D. Wang, "Relaxation oscillators with time delay coupling," Physica D 111, 151-178 (1998).

Online Version 7

[34] N. Shanks, "Modeling biological systems: The Belousov-Zhabotinsky reaction," Foundations of Chemistry 3, 33-53 (2001).

Online Version 9

[35] M. R. Tinsley, A. F. Taylor, Z. Huang, and K. Showalter, "Emergence of collective behavior in groups of excitable catalyst-loaded particles: Spatiotemporal dynamical quorum sensing." Phys. Rev. Lett. 102, 158301 (2009).

Online Version 9

[36] J. Maselko and K. Showalter, "Chemical waves on spherical surfaces," Nature 339, 609-611 (1989).

Online Version 9

[37] E. Mihaliuk, R. Wackerbauer, and K. Showalter, "Topographic organization of Hebbian neural connections by synchronous wave activity." Chaos 11, 287-292 (2001).

Online Version 9

[38] A. F. Taylor, P. Kapetanopoulos, B. J. Whitaker, R. Toth, L. Bull, and M. R. Tinsley, "Phase clustering in globally coupled photochemical oscillators," Eur. Phys. J. Spec. 165, 137-149 (2008).

Online Version 9

[39] P. Jung, A. Cornell-Bell, F. Moss, S. Kadar, J. Wang, and K. Showalter, "Noise sustained 
waves in subexcitable media: From chemical waves to brain waves." Chaos 8, 567-575 (1998).

Online Version 9 


\section{Chapter 2}

\section{The Belousov-Zhabotinsky Chemistry}

\section{and Models}

\section{$2.1 \quad$ Introduction}

In this study of coupled oscillators, the chemistry of the Belousov-Zhabotinsky reaction [1] will be used. Coupling will be achieved using light-based feedback. The experimental work will be supported by simulations using the Zhabotinsky-Buchholtz-Kiyatin-Epstein (ZBKE) model [2] to represent the BZ chemistry. Phase response curves, descriptions of the dynamical behavior in coupled popluations of oscillators, will be discussed.

\subsection{The Belousov-Zhabotinsky Reaction}

The Belousov-Zhabotinsky reaction was named after B. Belousov who initially discovered the reaction in 1958 and A. M. Zhabotinsky who later on further studied the reaction [1]. The reaction is now commonly referred to as the BZ reaction. The reaction involves the oxidation of organic 
species by bromate in the presence of a metal catalyst. The reaction has a complex mechanism involving a number of steps forming many different reaction intermediates. In 1972, R. J. Field, E. Körös, and R. M. Noyes proposed the FKN mechanism for the BZ reaction [3]. A number of models have since been developed and successfully applied in various studies to explain the experimental behavior of the system; these include the Oregonator model proposed by Field and Noyes [4] and the ZBKE model proposed by Zhabotinsky, Buchholtz, Kiyatin, and Epstein [2].

\subsubsection{The FKN Mechanism}

The mechanism features three main processes, A, B, and C, which are the consumption of bromide ions, the autocatalytic production of bromous acid, and the oxidation of organic species, respectively. The detailed mechanism consists of eleven elementary steps that can be summarized by the net equations

Process A (removal of bromide ions):

$$
\mathrm{BrO}_{3}^{-}+2 \mathrm{Br}^{-}+3 \mathrm{H}^{+} \rightarrow 3 \mathrm{HOBr}
$$

Process B (autoctalysis):

$$
\mathrm{BrO}_{3}^{-}+\mathrm{HBrO}_{2}+2 \mathrm{M}^{2+}+3 \mathrm{H}^{+} \rightarrow 2 \mathrm{HBrO}_{2}+2 \mathrm{M}^{3+}+\mathrm{H}_{2} \mathrm{O}
$$

Process C (oxidation of organic species):

$$
2 \mathrm{M}^{3+}+\mathrm{MA}+\mathrm{BrMA} \rightarrow \mathrm{fBr}^{-}+2 \mathrm{M}^{2+}+\text { other products }
$$

where $\mathrm{M}$ is the metal catalyst and $\mathrm{f}$ is a stoichiometric factor. In an elementary step that contributes to process $\mathrm{A}, \mathrm{Br}^{-}$reacts with $\mathrm{BrO}_{3}^{-}$and its concentration falls to a critical concentration. The low bromide concentration allows the autocatalytic production of $\mathrm{HBrO}_{2}$, as described in process 
B. This is accompanied by the oxidation of the catalyst $\left(\mathrm{M}^{2+} \rightarrow \mathrm{M}^{3+}\right)$. The increase in $\mathrm{HBrO}_{2}$ concentration is limited by the reaction

$$
2 \mathrm{HBrO}_{2} \rightarrow \mathrm{HBrO}_{3}+\mathrm{HOBr}
$$

The presence of $\mathrm{M}^{3+}$ gives rise to the oxidation of bromomalonic acid to form bromide ion and other products (process $\mathrm{C}$ ). The production of bromide ions in process $\mathrm{C}$ resets the reaction back to process A.

\subsubsection{The ZBKE Reaction Scheme and Model}

The ZBKE reaction scheme is based on the original FKN mechanism, processes A, B, and C. The mechanism includes more of the detailed chemistry of step $\mathrm{C}$, including the reactions of $\mathrm{HBrO}_{2}{ }^{+}$and organic species. The detailed mechanism is given in Table 2.1. In the table, $\mathrm{M}^{n+}$ and $\mathrm{M}^{(n+1)}$ represent the reduced and oxidized forms of the catalyst $[2,5]$. Reactions of $\mathrm{HOBr}$ and $\mathrm{Br}_{2}$ proceed rapidly and are therefore neglected in the description. The system is simplified by considering the organic molecules, $\mathrm{CBr}_{2}(\mathrm{COOH})_{2}, \mathrm{CHOH}(\mathrm{COOH})_{2}$, and $\mathrm{CO}(\mathrm{COOH})_{2}$ as final products and assuming the equilibrium in reaction $\mathrm{R} 5 \mathrm{c}$ is shifted to the right [2]. The mathematical model based on the mechanism is given by

$$
\begin{gathered}
\frac{d X}{d t}=-k_{2} h_{0} X Y+k_{3} h_{0} X Y-2 k_{4}^{*} X^{2}-k_{5} h_{0} A X+k_{-5} U^{2}+k_{6} U(C-Z)-k_{-6} X Z \\
\frac{d Y}{d t}=-k_{2} h_{0} X Y-k_{3} h_{0} A Y+k_{8}^{\prime} R_{1}+k_{9} B \\
\frac{d U}{d t}=2 k_{5} h_{0} A X-2 k_{-5} U^{2}-k_{6} U(C-Z)+k_{-6} X Z \\
\frac{d Z}{d t}=k_{6} U(C-Z)-k_{-6} X Z-k_{7} B Z+k_{-7} h_{0} R_{1}(C-Z) \\
\frac{d R_{1}}{d t}=k_{7} B Z-k_{-7} h_{0} R_{1}(C-Z)-k_{8}^{\prime} R_{1}-k_{11} R_{1} R_{2}
\end{gathered}
$$


Table 2.1. The ZBKE Reaction Scheme [2]

\begin{tabular}{|c|c|}
\hline $\mathrm{H}^{+}+\mathrm{Br}^{-}+\mathrm{HOBr} \rightleftharpoons \mathrm{Br}_{2}+\mathrm{H}_{2} \mathrm{O}$ & (R1) \\
\hline $\mathrm{H}^{+}+\mathrm{Br}^{-}+\mathrm{HBrO}_{2} \rightleftharpoons 2 \mathrm{HOBr}$ & $(\mathrm{R} 2)$ \\
\hline $\mathrm{H}^{+}+\mathrm{Br}^{-}+\mathrm{HBrO}_{3} \rightleftharpoons \mathrm{HBrO}_{2}+\mathrm{HOBr}$ & (R3) \\
\hline $2 \mathrm{HBrO}_{2} \rightleftharpoons \mathrm{HOBr}+\mathrm{HBrO}_{3}$ & $(\mathrm{R} 4 \mathrm{a})$ \\
\hline $\mathrm{HBrO}_{2}+\mathrm{H}^{+} \rightleftharpoons \mathrm{H}_{2} \mathrm{BrO}_{2}^{+}$ & $(\mathrm{R} 4 \mathrm{~b})$ \\
\hline $\mathrm{HBrO}_{2}+\mathrm{H}_{2} \mathrm{BrO}_{2}^{+} \rightleftharpoons \mathrm{HOBr}+\mathrm{HBrO}_{3}+\mathrm{H}^{+}$ & $(\mathrm{R} 4 \mathrm{c})$ \\
\hline $\mathrm{H}^{+}+\mathrm{BrO}_{3}^{-} \rightleftharpoons \mathrm{HBrO}_{3}$ & $(\mathrm{R} 5 \mathrm{a})$ \\
\hline $\mathrm{HBrO}_{3}+\mathrm{H}^{+}+\mathrm{HBrO}_{2} \rightleftharpoons \mathrm{HBrO}_{2}^{+}+\mathrm{BrO}_{2}+\mathrm{H}_{2} \mathrm{O}$ & $(\mathrm{R} 5 \mathrm{~b})$ \\
\hline $\mathrm{BrO}_{2}+\mathrm{H}^{+} \rightleftharpoons \mathrm{HBrO}_{2}^{+}$ & $(\mathrm{R} 5 \mathrm{c})$ \\
\hline $\mathrm{M}^{n+}+\mathrm{HBrO}_{2}^{+} \rightleftharpoons \mathrm{M}^{(n+1)}+\mathrm{HBrO}_{2}$ & $(\mathrm{R} 6)$ \\
\hline $\mathrm{M}^{(n+1)}+\mathrm{CHBr}(\mathrm{COOH})_{2} \rightleftharpoons \mathrm{M}^{n+}+\mathrm{CBr}(\mathrm{COOH})_{2}+\mathrm{H}^{+}$ & $(\mathrm{R} 7)$ \\
\hline $\mathrm{H}_{2} \mathrm{O}+\mathrm{CBr}(\mathrm{COOH})_{2} \rightarrow \mathrm{H}^{+}+\mathrm{Br}^{-}+\mathrm{COH}(\mathrm{COOH})_{2}$ & $(\mathrm{R} 8)$ \\
\hline $\mathrm{H}_{2} \mathrm{O}+\mathrm{CHBr}(\mathrm{COOH})_{2} \rightarrow \mathrm{CHOH}(\mathrm{COOH})_{2}+\mathrm{H}^{+}+\mathrm{Br}^{-}$ & (R9) \\
\hline $2 \mathrm{COH}(\mathrm{COOH})_{2} \rightarrow \mathrm{CHOH}(\mathrm{COOH})_{2}+\mathrm{CO}(\mathrm{COOH})_{2}$ & $(\mathrm{R} 10)$ \\
\hline $\mathrm{COH}(\mathrm{COOH})_{2}+\mathrm{CBr}(\mathrm{COOH})_{2} \rightarrow \mathrm{CHBr}(\mathrm{COOH})_{2}+\mathrm{CO}(\mathrm{COOH})_{2}$ & $(\mathrm{R} 11)$ \\
\hline $\mathrm{HOBr}+\mathrm{CHBr}(\mathrm{COOH})_{2} \rightarrow \mathrm{CBr}_{2}(\mathrm{COOH})_{2}+\mathrm{H}_{2} \mathrm{O}$ & $(\mathrm{RA}-1)$ \\
\hline $\mathrm{Br}_{2}+\mathrm{CHBr}(\mathrm{COOH})_{2} \rightarrow \mathrm{CBr}_{2}(\mathrm{COOH})_{2}+\mathrm{H}^{+}+\mathrm{Br}^{-}$ & $(\mathrm{RA}-2)$ \\
\hline
\end{tabular}




$$
\frac{d R_{2}}{d t}=k_{8}^{\prime} R_{1}-2 k_{10} R_{2}^{2}-k_{11} R_{1} R_{2}
$$

where, $X=\left[\mathrm{HBrO}_{2}\right], Y=\left[\mathrm{Br}^{-}\right], U=\left[\mathrm{HBrO}_{2}^{+}\right], Z=\left[\mathrm{M}^{(n+1)}\right], R_{1}=\left[\mathrm{CBr}(\mathrm{COOH})_{2}\right], R_{2}=$ $\left[\mathrm{COH}(\mathrm{COOH})_{2}\right], A=\left[\mathrm{HBrO}_{3}\right]=h_{0}\left[\mathrm{NaBrO}_{3}\right]_{0} /\left(0.2+h_{0}\right), B=\left[\mathrm{CHBr}(\mathrm{COOH})_{2}\right], C=Z+\left[\mathrm{M}^{n+}\right]$, $h_{0}=$ Hammet acidity function, $k_{4}^{*}=k_{4}\left(1+0.8 h_{0}\right), k_{-5}$ is represented by $\left(k_{-5 b} k_{-5 c}\right) /\left(k_{5 c} h_{0}\right)$. By assuming fast radical recombination, reactions $\mathrm{R} 10$ and R11, and using the quasi-steady-state approximation to exclude R2, equation 2.10 for R1 simplifies to:

$$
\frac{d R_{1}}{d t}=k_{7} B Z-k_{-7} h_{0} R_{1}(C-Z)-\frac{k_{8}^{\prime} R_{1}}{q\left(R_{1}\right)}
$$

where

$$
\frac{1}{q\left(R_{1}\right)}=1-\frac{k_{11}^{2} R_{1}}{4 k_{8}^{\prime} k_{10}}\left\{1-\left(1+\frac{8 k_{8}^{\prime} k_{10}}{k_{11}^{2} R_{1}}\right)^{1 / 2}\right\} .
$$

A parameter $q=q(R 1)$ is introduced, which can be varied from 0.5 to 1 , and $k_{8}$ is set to $k_{8}^{\prime} / q$. The lower and upper values correspond to the conditions when reaction R11 is faster than R10 and when reaction R10 is faster than R11, respectively [2]. The model is reduced to a three-variable system by assuming the concentration of $\left[\mathrm{CBr}(\mathrm{COOH})_{2}\right]$ is a fast variable and treating $\left[\mathrm{HBrO}_{2}^{+}\right]$ as a steady state variable $\left(U_{s s}\right)$.

$$
\begin{gathered}
\frac{d X}{d t}=\frac{-k_{2} X+k_{3} A}{k_{2} X+k_{3} A}\left(q k_{7} k_{8} P+k_{9} B\right)-2 k_{4}^{*} X^{2}-k_{5} h_{0} A X+k_{-5} U_{s s}^{2}+k_{6} U_{s s}(C-Z)-k_{-6} X Z \\
\frac{d Y}{d t}=-k_{2} h_{0} X Y-k_{3} h_{0} A Y+q P P+k_{9} B \\
\frac{d Z}{d t}=k_{6} U_{s s}(C-Z)-k_{-6} X Z-P P .
\end{gathered}
$$

where

$$
\begin{gathered}
U_{s s}=\frac{1}{4 k_{-5}}\left(k_{-6}(C-Z)+\left(k_{-6}^{2}(C-Z)^{2}+16 k_{-5} k_{5} h_{0} A x+8 k_{-5} k_{-6} x z\right)^{2}\right), \\
P=\frac{B Z}{k_{8}+k_{-7} h_{0} R_{1}(C-Z)},
\end{gathered}
$$


Table 2.2. The ZBKE Model Scalings $[2,6]$

\begin{tabular}{|c|c|c|c|c|c|}
\hline Variable & Scaling & Parameter & Scaling & Parameter & Scaling \\
\hline$X$ & $\mathrm{k}_{5} h_{0} A x / 2 k_{4}$ & $\varepsilon_{1}$ & $\mathrm{k}_{5} h_{0} A / 2 k_{4} C$ & $\beta$ & $2 \mathrm{k}_{4} k_{9} B /\left(k_{5} h_{0} A\right)^{2}$ \\
$Y$ & $\mathrm{k}_{5} A y / k_{2}$ & $\varepsilon_{2}$ & $\left(\mathrm{k}_{5} h_{0} A\right)^{2} / 2 k_{4} k_{6} C$ & $\mu$ & $2 \mathrm{k}_{3} k_{4} B / k_{2} k_{5} h_{0}$ \\
$Z$ & $\mathrm{Cz}$ & $\varepsilon_{3}$ & $\mathrm{k}_{8} / k_{-7} h_{0} C$ & $\gamma$ & $\mathrm{k}_{-5} / k_{6}$ \\
$U$ & $\left(\mathrm{k}_{5} h_{0} A\right)^{2} u / 2 k_{4} k_{6} C$ & $\varepsilon_{4}$ & $\mathrm{k}_{5} A / k_{2} C$ & $\delta$ & $\mathrm{k}_{-6} C / k_{5} h_{0} A$ \\
$t$ & $2 \mathrm{k}_{4} C \tau /\left(k_{5} h_{0} A\right)^{2}$ & $\alpha$ & $2 \mathrm{k}_{4} k_{7} k_{8} B / k_{4}^{2} k_{-7} h_{0}^{3} A^{2}$ & $k$ & $2 \mathrm{k}_{4} C K /\left(k_{5} h_{0} A\right)^{2}$ \\
\hline
\end{tabular}

and

$$
P P=\frac{k_{7} k_{8} B Z}{k_{8}+k_{-7} h_{0}(C-Z)}
$$

The equations are scaled appropriately to a dimensionless model, which allows a qualitative comparison with experiments. The nondimensional equations for the ferroin catalyzed reaction are given in equations $(2.19)$ - $(2.21)[2,6]$. The scaling of variables and parameters are given in Table 2.2 .

$$
\begin{gathered}
\frac{d x}{d \tau}=\frac{1}{\varepsilon_{1}}\left(\varphi-x^{2}+x+\varepsilon_{2} \gamma u_{s s}^{2}+u_{s s}(1-z)+\delta x z+\frac{\mu-x}{\mu-z}\left(\frac{q \alpha z}{\varepsilon_{3}+1-z}+\beta\right)\right) \\
\frac{d y}{d \tau}=\frac{1}{\varepsilon_{4}}\left(-x y-\mu y+q \frac{\alpha z}{\varepsilon_{3}+1-z}+\beta\right) \\
\frac{d z}{d \tau}=2 \varphi+u_{s s}(1-z)-\delta x z-\frac{\alpha z}{\varepsilon_{3}+1-z}
\end{gathered}
$$

The three-variable model can be further reduced to a two-variable model by using a steady approximation of $y$. The principal advantage of using the ZBKE model over the traditional Oregonator model is that the ZBKE produces significant frequency variations with a change in the parameter q. This feature is important for simulating the behavior of heterogeneous populations of chemical oscillators in experiments. 


\subsubsection{Photosensitive BZ Reaction System}

The ruthenium-catalyzed BZ reaction has been widely used in feedback control dynamical systems [7-14]. The sensitivity to light of the tris(2,2'-bipyridine)ruthenium(II) complex, $\left(\mathrm{Ru}(\mathrm{bpy})_{3}^{2+}\right)$, can be used to control the concentration of reacting species in the ruthenium catalyzed BZ reaction.

The complex can be excited to $\mathrm{Ru}(\mathrm{bpy})_{3}^{2+*}$ by light at $452 \mathrm{~nm}[15,16]$. The excited $\mathrm{Ru}(11)^{*}$ species is a strong reducing agent as indicated by a reduction potential of $-0.86 \mathrm{~V}$ for $\mathrm{Ru}(\mathrm{lll}) / \mathrm{Ru}(\mathrm{ll})$ system [15, 17]. Kadár et al. [15] determined two separate reaction pathways of the excited ruthenium complex that may occur at low and high light intensities. At low light intensity, the excited species react with bromate ions producing $\mathrm{BrO}_{2}$, which further reacts with $\mathrm{Ru}(l \mathrm{l})$ to give bromous acid and $\mathrm{Ru}(\mathrm{lll})$, as represented by equations [15]

$$
\begin{gathered}
\mathrm{Ru}(\mathrm{ll})^{*}+\mathrm{BrO}_{3}^{-}+2 \mathrm{H}^{+} \rightarrow \mathrm{Ru}(\mathrm{ll})+\mathrm{BrO}_{2}+\mathrm{H}_{2} \mathrm{O} \\
\mathrm{Ru}(\mathrm{ll})^{*}+\mathrm{BrO}_{2}+\mathrm{H}^{+} \rightarrow \mathrm{Ru}(\mathrm{lll})+\mathrm{HBrO}_{2}
\end{gathered}
$$

Both $\mathrm{Ru}(\mathrm{lll})$ and the activator, $\mathrm{HBrO}_{2}$, are important components of the ZBKE model described in the next section. At high light intensity, the inhibitor is produced from the reaction of the excited species with bromomalonic acid [15]

$$
\mathrm{Ru}(\mathrm{ll})^{*}+\mathrm{BrMA} \rightarrow \mathrm{Ru}(\mathrm{lll})+\mathrm{Br}^{-}+\text {organic products }
$$

Oscillations are completely suppressed above a critical illumination intensity [15, 17] because of the photochemical production of the inhibitor. The oscillation period of the system increases with increase in bromide concentration, as it takes more time for the bromide concentration, to fall below the critical concentration to allow the autocatalytic process to take over. Sensitivity to light depends also on the initial concentrations of reactants [15, 17]. Taylor et al. [17] found that higher bromate and acid concentration relative to malonic acid and bromide tends to follow the 
photoactivatory pathway, while a higher ratio of the inhibitory species compared to the activatory species favors the photoinhibitory pathway.

\subsubsection{The Modified ZBKE Model}

The ZBKE model described in Section 2.2.2 can be modified to account for the rutheniumbased photosensitive behavior of the BZ reaction. The ruthenium catalyst exhibits kinetics that is different from ferroin in reactions leading to the autocatalytic reaction in processes B of the FKN mechanism. The reaction of the $\mathrm{Ru}(\mathrm{II})$ complex with $\mathrm{BrO}_{2}$ has a large rate constant [17]

$$
\mathrm{BrO}_{2}+\mathrm{Ru}(\mathrm{bpy})_{3}^{2+}+\mathrm{H}^{+} \rightarrow \mathrm{HBrO}_{2}+\mathrm{Ru}(\mathrm{bpy})_{3}^{3+}, \quad \mathrm{k}=4 \times 10^{6} \mathrm{~m}^{-2} \mathrm{~s}^{-1}
$$

The model is modified to account for the irreversibility of this reaction step. A term, $\phi$, which was introduced by Vanag et al. [18] as a $\mathrm{Br}^{-}$flow term, is introduced in the model to account for photoinhibition. Further studies $[15,17]$ have led to modifications to the model to include $\phi$ to account for photoactivation. The modified dimensionless two-variable photosensitive ZBKE model is represented by rate equations of the autocatalyst $\mathrm{HBrO}_{2}(x)$ and oxidized form of the catalyst $\mathrm{Ru}(\mathrm{bpy})_{3}^{3+}(z)$ given by

$$
\begin{gathered}
\frac{d x}{d \tau}=\frac{1}{\varepsilon_{1}}\left(\varphi-x^{2}+x+\varepsilon_{2} \gamma u_{s s}^{2}+u_{s s}(1-z)+\frac{\mu-x}{\mu-z}\left(\frac{q \alpha z}{\varepsilon_{3}+1-z}+\beta\right)\right) \\
\frac{d z}{d \tau}=2 \varphi+u_{s s}(1-z)-\frac{\alpha z}{\varepsilon_{3}+1-z}
\end{gathered}
$$

where $u_{s s}$ is the scaled steady-state variable $\left[\mathrm{HBrO}_{2}^{+}\right]$. The parameters contain the rate constants, initial concentrations and the appropriate scalings for the model variables. The parameter values used in simulations are given in Table 2.3. All calculations are performed using the Euler method with step $0.001, \phi_{0}=5.3 \times 10^{-4}$ and $\phi_{\max }=2.1 \times 10^{-4}$. Figure 2.1 shows a phase plot of dimensionless variables $[z]$ vs $[x]$, and typical concentration variations of $x$ and $z$ with time. 
Table 2.3. The ZBKE Model Parameter Values [11-14]

\begin{tabular}{|c|c|c|c|}
\hline Parameter & Value & Parameter & Value \\
\hline$\epsilon_{1}$ & 0.11 & $\alpha$ & 0.10 \\
$\epsilon_{2}$ & $1.7 \times 10^{-5}$ & $\beta$ & $1.7 \times 10^{-5}$ \\
$\epsilon_{3}$ & $1.6 \times 10^{-3}$ & $\mu$ & $2.4 \times 10^{-4}$ \\
$\gamma$ & 1.2 & $q$ & $41 \pm 2.1$ \\
\hline
\end{tabular}
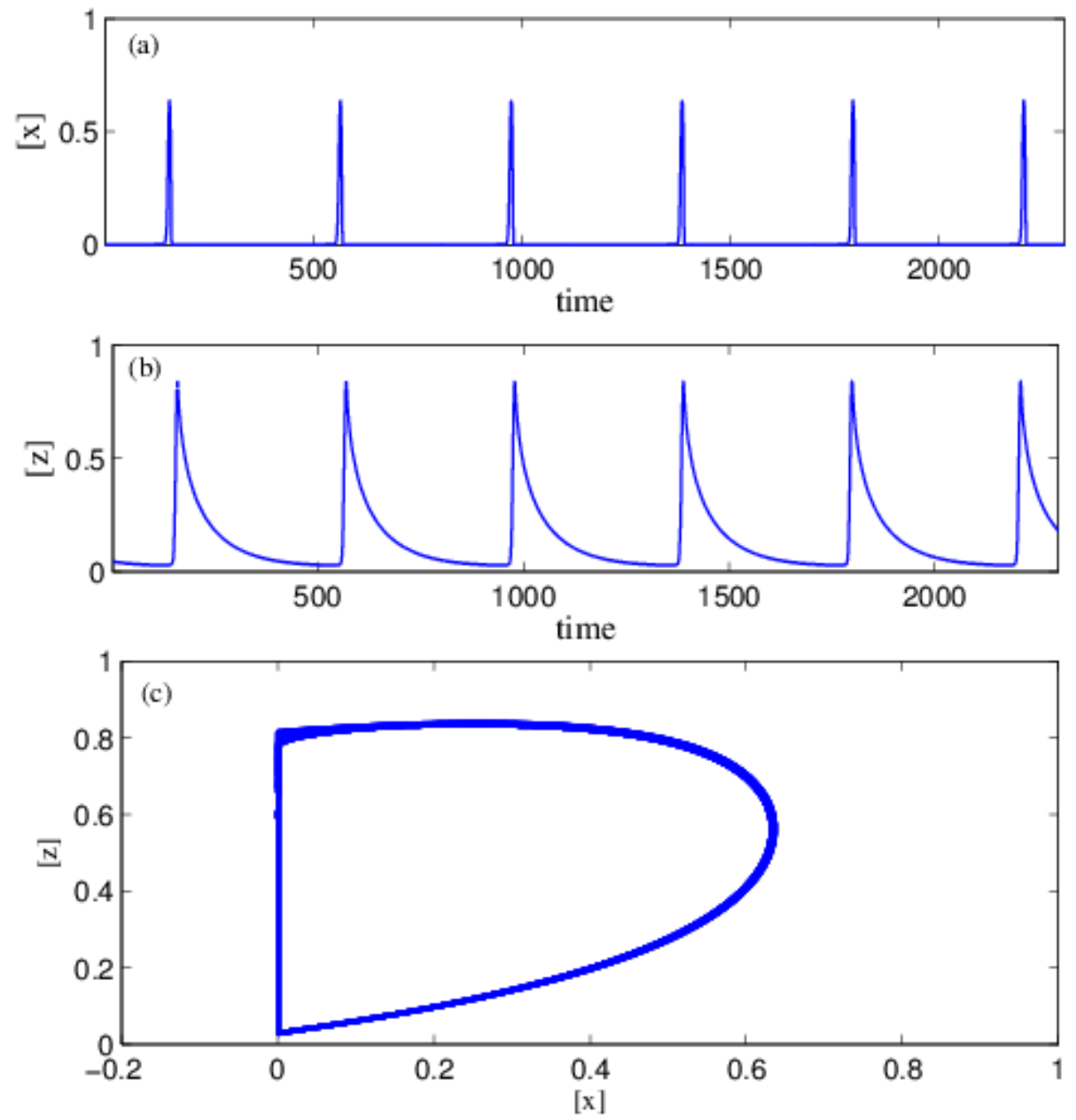

Figure 2.1. (a) Time series of the activator species $x$ and (b) time series of the oxidized form of catalyst $z$ from model simulations. (c) Phase plot $[z]$ vs $[x]$. 


\subsection{Experiment Setup}

\subsubsection{Instrumentation}

The experimental set-up consists of a modified projector (SLM) with a 440-460 nm band pass filter, beam splitter, camera (CCD), computer (PC), and the reactor. The camera and the modified projector are controlled via the computer. The camera is placed above the beam splitter and a 2 $\mathrm{cm}$ diameter reaction vessel to record images of the state of the reaction domain in real time. The set-up is shown in Figure 2.2.

a)

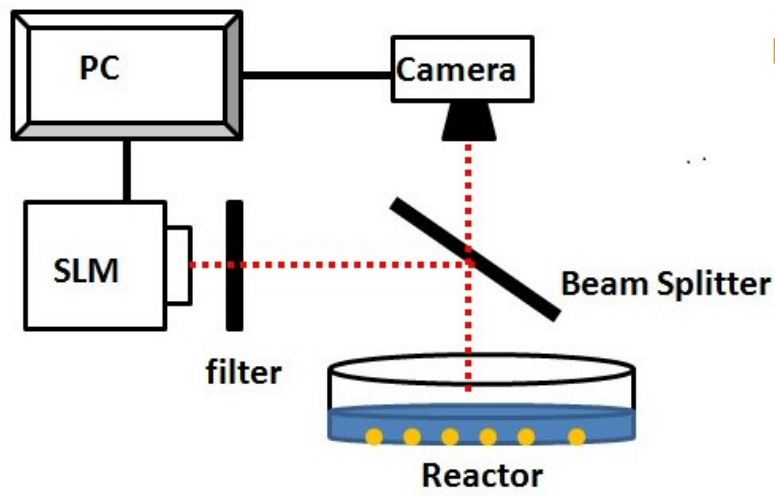

b)

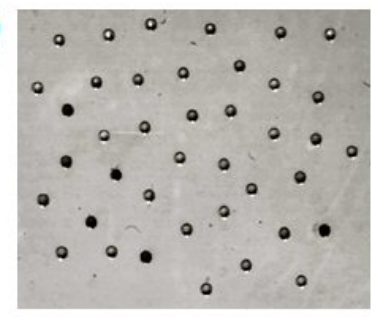

Figure 2.2. Experiment set-up. The camera (CCD), connected to the computer (PC), records the position and intensity of the catalyst-loaded beads in the reactor. The image is processed by the PC and feedback is applied via the spatial light modulator (SLM) through the filter. (b) Catalyst-loaded particles recorded by the CCD camera.

The CCD camera is connected to a PC, which processes the images and computes the feedback illumination for each particle. The feedback illumination is applied through a spatial light modulator (SLM). The light from the SLM passes through the band-pass filter to a beam splitter that reflects the light on to the particles in the reactor. 


\subsubsection{Preparation of Ruthenium(ll) Catalyst-Loaded Beads}

The catalyst solution of $25.0 \mathrm{mM} \mathrm{Ru}(\mathrm{bpy})_{3}{ }^{2+}$ is prepared by dissolving $0.47 \mathrm{~g}$ of tris $(2-2$ 'bipyridyl)ruthenium(II) chloride hexahydrate (98\% complex) in $25.0 \mathrm{ml}$ of doubly-distilled water. DOWEX 50wx2-100 ion exchange beads $(3.0 \mathrm{~g})$ are mixed with $1.0 \mathrm{ml}$ of $25.0 \mathrm{mM}$ ruthenium(ll) catalyst solution $\left(8.3 \times 10^{-6} \mathrm{~mol} / \mathrm{g}\right.$ resin $)$. The mixture is stirred at a low stirring rate for 24 hours and then filtered. The beads are washed with distilled water before allowing them to dry at room temperature for a day.

\subsubsection{Preparation of Catalyst-Free BZ Solution}

Stock solutions of 1.0 M sodium bromide $(\mathrm{NaBr}), 1.5 \mathrm{M}$ sodium bromate $\left(\mathrm{NaBrO}_{3}\right)$, and 3.0 M sulfuric acid $\left(\mathrm{H}_{2} \mathrm{SO}_{4}\right)$, from Fischer Scientific, are prepared with doubly distilled water. The solutions are stored at room temperature. Malonic acid (MA) is always prepared before the start of an experiment because it is not stable over periods greater than approximately 24 hours. The catalyst free BZ solution is prepared by mixing $0.77 \mathrm{M} \mathrm{H}_{2} \mathrm{SO}_{4}, 0.51 \mathrm{M}\left(\mathrm{NaBrO}_{3}\right), 0.08 \mathrm{M} \mathrm{NaBr}$ and 0.16 M MA.

\subsubsection{Experiment}

The catalyst-loaded particles are randomly distributed in a BZ catalyst-free solution. The particles are maintained at more than two diameters apart to eliminate local diffusive coupling. The particles are in an oscillatory state under the experimental conditions. Experiments are carried out with 40 particles. A computer algorithm is used to align the camera and projector coordinate systems to allow real-time feedback by illumination of individual oscillators. The particles are mapped onto a virtual array, which is used to setup a desired network with a defined coupling 
relation. Different networks are discussed in Chapters 3, 4 and 5.

In experiments, the images are captured every $3.0 \mathrm{~s}$ with projected feedback turned off briefly to allow capturing of images. A background light intensity of $1.4 \mathrm{~mW} \mathrm{~cm}^{-2}$ is used when capturing images. Variations in transmitted light by individual particles are monitored over time. The observed oscillations in light intensity are due to the ruthenium(II) being oxidized to ruthenium(III) (green), which is then reduced back to ruthenium(II) (orange). The oxidized form of the catalyst transmits more light than the reduced form. Thus, the peak maximum corresponds to the oxidized form of the ruthenium catalyst. The captured image is analyzed to determine the intensities, in gray level, associated with each particle. The intensities are used to compute the feedback based on the coupling scheme. The computed illumination is passed on to the projector, which illuminates particles independently according to

$$
P_{i}=P_{0}+P_{\text {feed }, i}
$$

where $P_{i}$ is the projected light intensity, $P_{0}$ is the background light intensity, and $P_{f e e d, i}$ is the computed feeback for oscillator $i[19]$. $P_{\text {feed }, i}$ is computed according to a coupling relation defined for each network studied.

The data on changes in light intensity over time is stored for further analysis. Figure 2.3 shows typical time series from experiment data. 


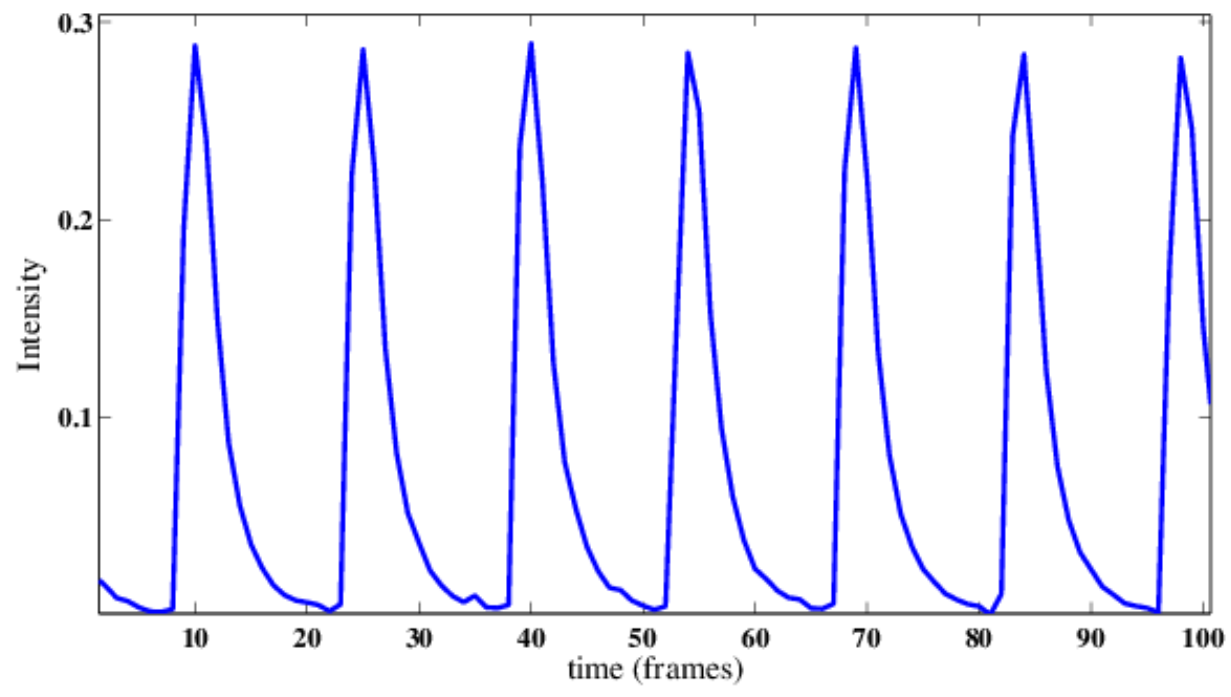

Figure 2.3. Experiment time series

\subsection{Phase Response Curves}

\subsubsection{Introduction}

A phase response curve quantifies an oscillator's response to perturbations. Phase response curves (PRC) play a pivotal role in explaining and predicting the dynamics of coupled oscillators [20]. They have been used to predict stability of various states in biological systems, including neuronal networks [21-25] and circadian rhythms [24, 26, 27]. They provide valuable information about an oscillator's response to a perturbation and its influence on its coupled neighbors, thereby influencing the global dynamics of a network. As highlighted by Dror et al. [22], the stability and synchronization behavior of a network is not only governed by the coupling relation but other factors like the slope of the PRC.

A PRC is determined by applying a brief pertubation to an oscillator at different phases within its cycle and then comparing the period of this cycle to the period of the unperturbed cycle. In our system, the phase of an oscillator can be manipulated by controling light exposure. This 
section focuses on effects of light pulses on photosensitive BZ relaxation oscillators. Taylor et al. $[17,19]$ studied the effect of light on both the photoexcitatory and photoinhibitory BZ mixtures by showing the dependence of the $\mathrm{PRC}$ on the composition of $\mathrm{BZ}$ mixtures. They observed a photoactivatory reaction pathway with mixtures that have higher bromate and acid concentrations relative to malonic acid and bromide species [17]. On the other hand, a higher ratio of inhibitory species compared to activatory species favors photoinhibitory pathway. Section 2.4.2 outlines an experimental determination of PRCs for the BZ mixture used in experiments with coupled oscillators that are described in the next chapters.

\subsubsection{Phase Response Experiments}

Phase response experiments were carried out using the photosensitive BZ reaction and the experiment design described in Section 2.3. Oscillators were evenly and randomly distributed, with more than two diameters apart, in a Petri dish. The oscillators were allowed to oscillate at a background light intensity $\left(\mathrm{P}_{0}\right)$. The average period of the oscillators is $60.0 \mathrm{~s}$. Different sized perturbations were applied every 300.0 s. The particles were perturbed by exposing them to a perturbation for $1.0 \mathrm{~s}$ and then switching back to the background intensity.

The phase $\varphi$ of an oscillator at any time was calculated using the equation

$$
\varphi(t)=\frac{2 \pi\left(t-t_{p e a k}\right)}{T}
$$

where $\mathrm{t}$ is the current time and $\mathrm{t}_{\text {peak }}$ is time of the previous peak. A response to a perturbation was determined by comparing the perturbed period to the unperturbed period of an oscillator. The response was calculated using the equation

$$
\Delta \varphi=\frac{2 \pi\left(T-T^{\prime}\right)}{T}
$$


where $\Delta \varphi$ is the phase response, $\mathrm{T}^{\prime}$ is the perturbed period, and $\mathrm{T}$ is the unperturbed period of the oscillator $[19,28]$. The $300.0 \mathrm{~s}$ interval between perturbations allowed the oscillators to 'relax' to their unperturbed behavior before applying another pulse. The mean period of the unperturbed cycles prior to each perturbation was used for $\mathrm{T}$ to account for drift, if any, in the system. The oscillators were perturbed 10 times in each experiment. The phase change values were calculated for each perturbation for all the oscillators, and then the values were averaged to obtain a better representation of the phases. A bin width of 0.4 was used and the corresponding mean phase response $\Delta \varphi$ was plotted for each bin.

\subsubsection{Results}

Phase advancement occurs when an oscillator's perturbed period is shorter than T, $\Delta \varphi>0$. On the other hand, phase retardation or delay occurs if the perturbed period is longer than $\mathrm{T}$, $\Delta \varphi<0$. Figure 2.4 shows examples, from experiments, of phase advancement, Figure $2.4 \mathrm{a}$, and phase retardation, Figure 2.4b. In Figure 2.4a, the oscillator has a period T equal to $69.0 \mathrm{~s}$ for $\mathrm{t} \leq$ $160.0 \mathrm{~s}$ and after perturbation the period shortens to $42.0 \mathrm{~s}(160.0 \leq \mathrm{t} \leq 212.0 \mathrm{~s})$. The oscillator in Figure 2.4b oscillates with a period T equal to $39.0 \mathrm{~s}$ for $\mathrm{t} \leq 138.0 \mathrm{~s}$ and was phase retarded on the fourth cycle to a period of $63.0 \mathrm{~s}$.

Phase response curves for the BZ experiment are shown in Figure 2.5a. A large perturbation

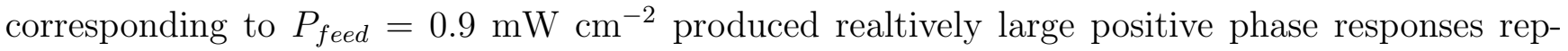
resented by the blue curve. Phase advancement was observed for perturbations applied between phase values of $\pi / 2$ and $2 \pi$. The maximum phase response, equivalent to approximately half a cycle, was produced at $0.75 \pi$. A smaller size perturbation of $P_{\text {feed }}=0.4 \mathrm{~mW} \mathrm{~cm}^{-2}$ caused phase advance responses to perturbations applied between $\pi$ and $2 \pi$. The magnitude of the maximum phase response was found to be approximately half of the maximum phase change produced by 


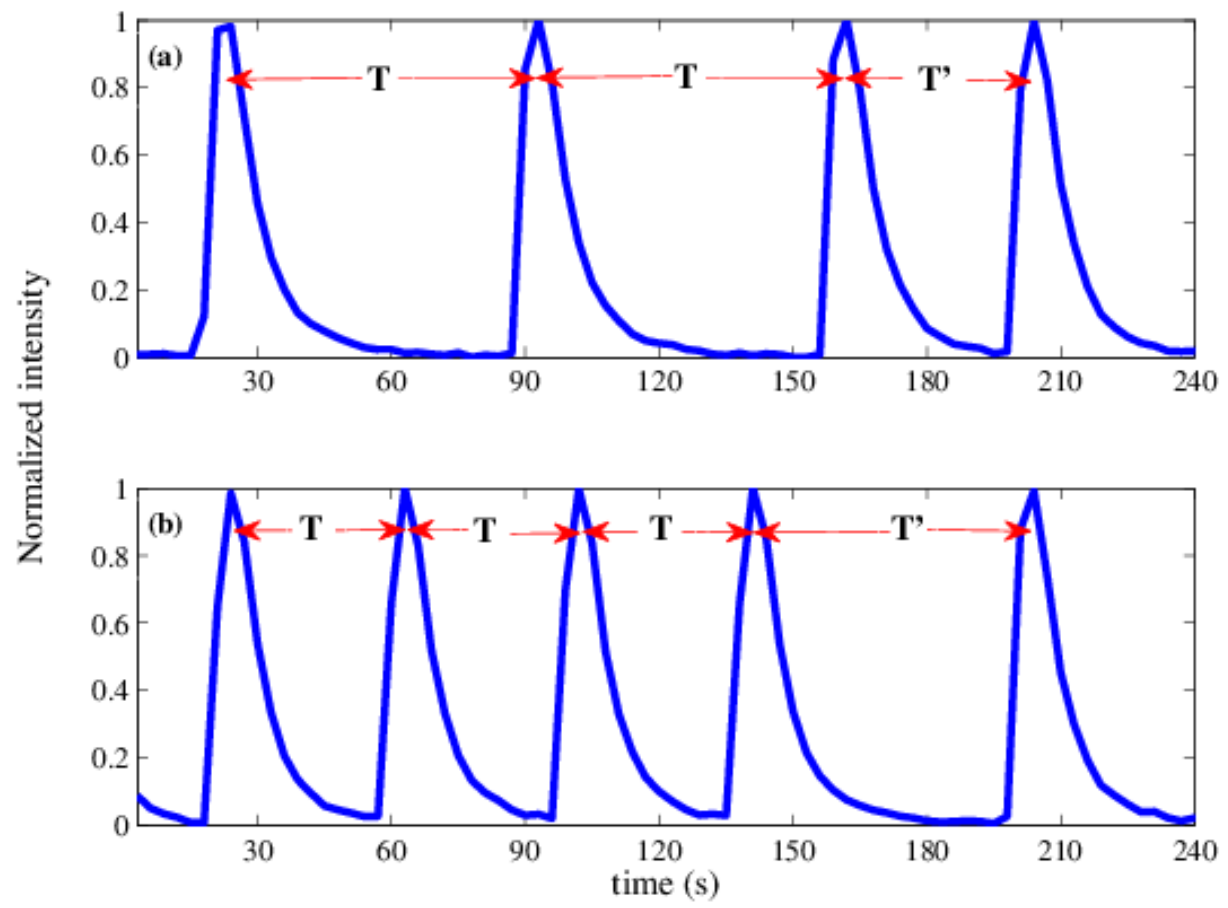

Figure 2.4. Experiment time series showing (a) phase advancement $\left(T>T^{\prime}\right)$ and (b) phase retardation $\left(\mathrm{T}<\mathrm{T}^{\prime}\right)$. $\mathrm{T}$ is the unperturbed period of the oscillator and $\mathrm{T}^{\prime}$ is the period after perturbation. 

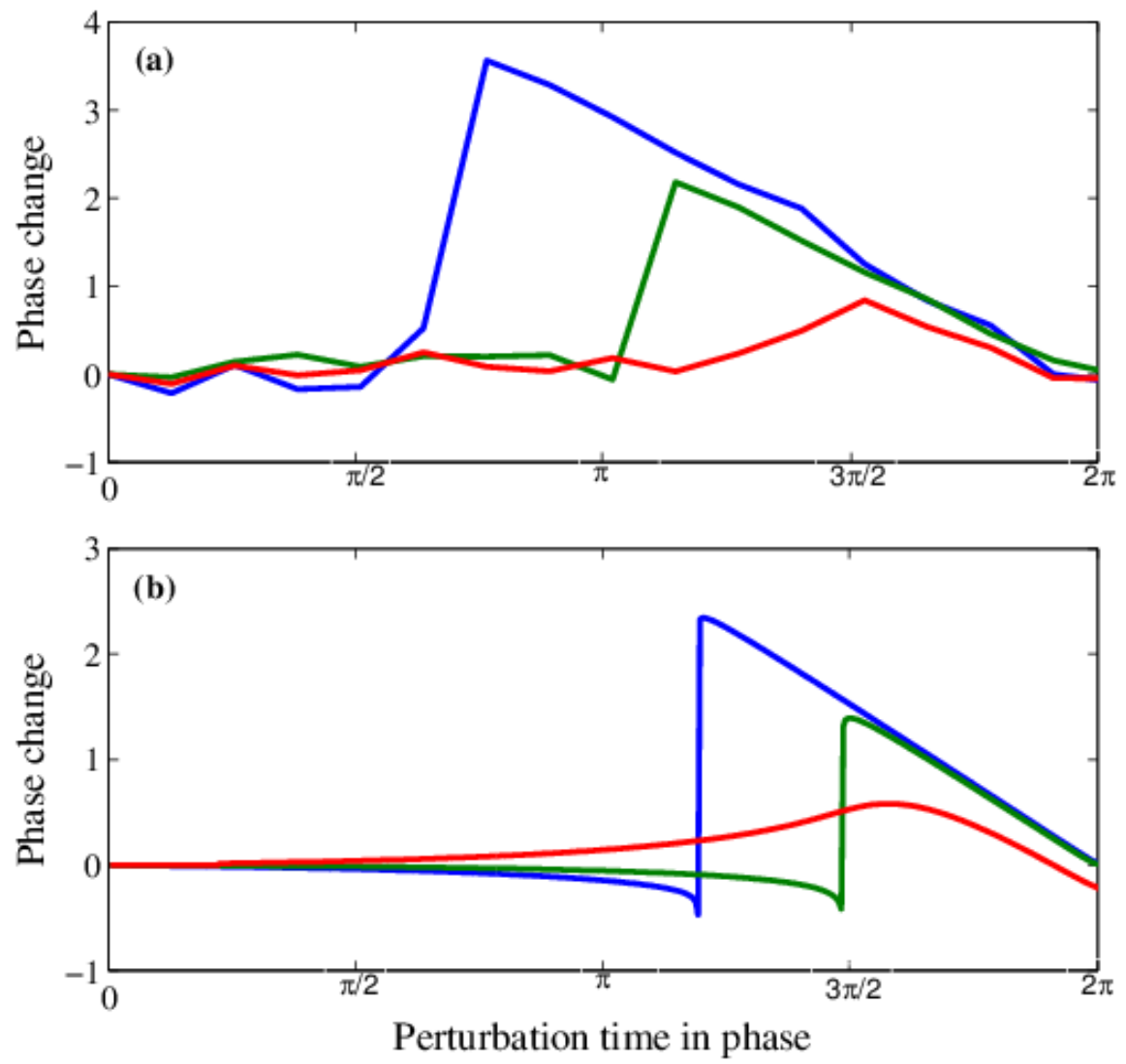

Figure 2.5. Phase response curves responding to different light perturbations. (a) Experiments with (i) $P_{\text {feed }}=0.9 \mathrm{~mW} \mathrm{~cm}^{-2}$, blue curve, (ii) $P_{\text {feed }}=0.4 \mathrm{~mW} \mathrm{~cm}^{-2}$, green curve, and (iii) $P_{\text {feed }}$ $=-0.9 \mathrm{~mW} \mathrm{~cm}^{-2}$, red curve. (b) Simulated response curves corresponding to (i) $P_{\text {feed }}=0.08$, blue curve, (ii) $P_{\text {feed }}=0.03$, green curve, and (i) $P_{\text {feed }}=-0.08$, red curve. 


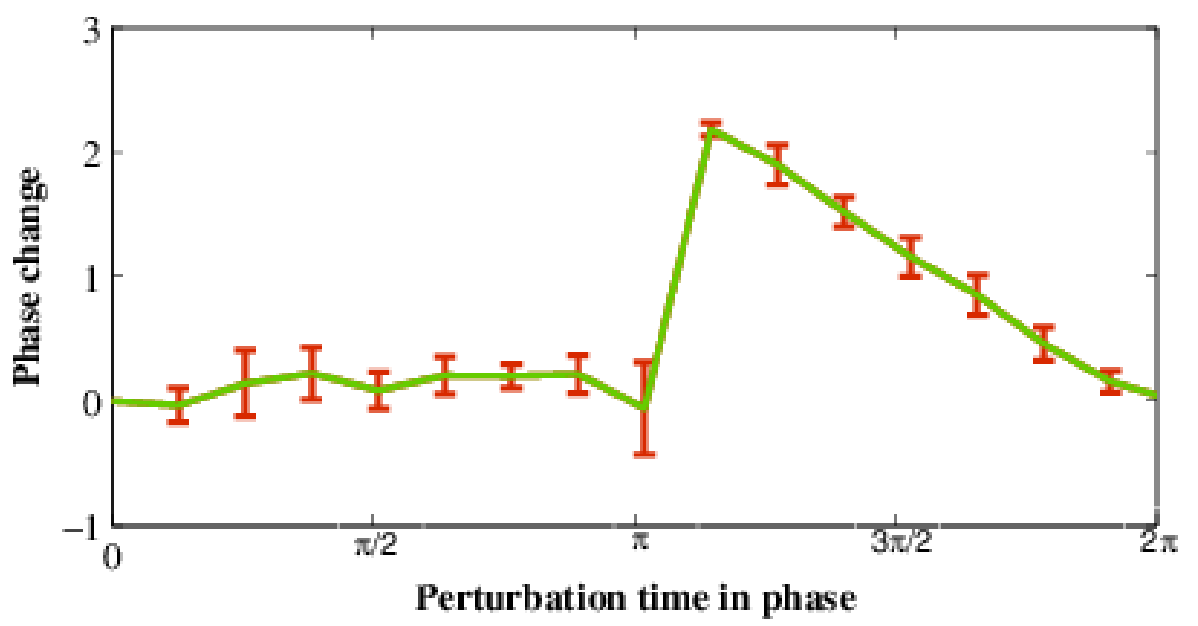

Figure 2.6. Phase response curve corresponding to a perturbation of $P_{\text {feed }}=0.4 \mathrm{~mW} \mathrm{~cm}{ }^{-2}$, green curve, showing error margin associated with each point.

$P_{\text {feed }}=0.9 \mathrm{~mW} \mathrm{~cm}^{-2}$ and it was observed at $\varphi=1.25 \pi$. Phase retardation was observed close to $\varphi=\pi$. For $P_{\text {feed }}=-0.9 \mathrm{~mW} \mathrm{~cm}^{-2}$, the oscillators were perturbed by reducing the light intensity from the background intensity. As represented by the red curve, a positive but smaller response similar to previous experiments was observed. The maximum phase change was produced at $1.5 \pi$. Similar observations were found in simulated PRCs, shown in Figure 2.5b. The simulated PRCs show relatively larger phase retardation regions for positive $P_{\text {feed }}$ perturbations compared to the experiments. This could be due to noise in experimental system. The experimental PRCs are produced from bin average values. The region of maximum phase retardation is close to the maximum phase advance region. The relatively large standard deviation shown in Figure 2.6 at phase $\pi$ suggests a possible overlap of advancement and retardation responses.

The simulated negative $P_{\text {feed }}$ perturbation shows a positive response similar to that observed in experiments. This could be a result of the photoactivatory nature of the experimental BZ mixture. The positive response observed is due to increased production of activator species following a light 
pulse, as described in Section 2.2.3 by equation 2.23. The rapid accumulation of $\mathrm{HBrO}_{2}$ is accompanied by the oxidation of $\mathrm{Ru}(\mathrm{II})$ to $\mathrm{Ru}(\mathrm{III})$. The reaction is accompanied by an increase of the oxidized form of the catalyst and is represented by a sharp increase in intensity in the time series. When perturbations are applied at earlier phases, negative responses are observed. This is due to $\mathrm{Ru}(\mathrm{III})$ being produced via production of $\mathrm{Br}^{-}$, and it takes longer for the bromide concentration to fall below the critical value to allow the autocatalytic process. Hence, this produces a longer period than an unperturbed period. Phase advancement and retardation occur at different phases due variations in the reaction mixture composition with phase. At some phases, the concentration of inhibitory species is relatively higher than at other phases. Oscillations were not signifantly affected following perturbations applied during the refractory period of the cycle.

In summary, the phase response depends on the nature and magnitude of perturbation, the phase of the oscillator when it is perturbed, and the composition of the reaction mixture. From the experiments and simulations described here, we find that increasing the size of a perturbation resulted in an increase in phase response, as noted in [28]. The observed global dynamics of coupled oscillators, discussed in the next chapters, is influenced by varying contributions of these factors. 


\section{References}

[1] A. M. Zhabotinsky, "A history of chemical oscillations and waves." Chaos 1, 379-386 (1991). Online Version 16

[2] A. M. Zhabotinsky, F. Bucholtz, A. B. Kiyatkin, and I. R. Epstein, "Oscillations and waves in metal-ion-catalyzed bromate oscillating reactions in highly oxidized states," J. Phys. Chem. 97, 7578-7584 (1993).

Online Version xi, 16, 17, 18, 19, 20, 21

[3] R. J. Field, E. Körös, and R. M. Noyes, "Oscillations in chemical systems. II. Thorough analysis of temporal oscillation in the bromate-cerium-malonic acid system," J. Am. Chem. Soc. 94, 8649-8664 (1972).

Online Version 17

[4] R. Field and R. M. Noyes, "Oscillations in chemical systems. IV. Limit cycle behavior in a model of a real chemical reaction," J. Chem. Phys. 60, 1877-84. (1974).

Online Version 17

[5] H. Sevcikova, I. Schreiber, and M. Marek, "Dynamics of oxidation Belousov-Zhabotinsky waves in an electric field," J. Phys. Chem. 100, 19153-19164 (1996).

Online Version 18

[6] R. Toth, A. F. Taylor, and M. R. Tinsley, "Collective behavior of a population of chemically coupled oscillators." J. Phys. Chem. B 110, 10170-10176 (2006).

Online Version xi, 21

[7] L. Yang, M. Dolnik, A. M. Zhabotinsky, and I. R. Epstein, "Oscillatory clusters in a model of the photosensitive Belousov-Zhabotinsky reaction system with global feedback," Phys. Rev. 
E 62, 6414-6420 (2000).

Online Version 22

[8] T. Sakurai, E. Mihaliuk, F. Chirila, and K. Showalter, "Design and control of wave propagation patterns in excitable media." Science 296, 2009-2012 (2002).

Online Version

[9] E. Mihaliuk, T. Sakurai, F. Chirila, and K. Showalter, "Feedback stabilization of unstable propagating waves.” Phys. Rev. E 65, 065602 (2002).

Online Version

[10] M. Hildebrand, J. Cui, E. Mihaliuk, J. Wang, and K. Showalter, "Synchronization of spatiotemporal patterns in locally coupled excitable media," Phys. Rev. E 68, 026205 (2003).

Online Version

[11] A. F. Taylor, P. Kapetanopoulos, B. J. Whitaker, R. Toth, L. Bull, and M. R. Tinsley, "Clusters and switchers in globally coupled photochemical oscillators." Phys. Rev. Lett. 100, 214101 (2008).

Online Version xi, 24

[12] A. F. Taylor, M. R. Tinsley, F. Wang, and K. Showalter, "Phase clusters in large populations of chemical oscillators," Angew. Chem. Int. Ed. 50, 10161-10164 (2011).

Online Version

[13] M. R. Tinsley, S. Nkomo, and K. Showalter, "Chimera and phase-cluster states in populations of coupled chemical oscillators," Nature Physics 8, 662-665 (2012).

Online Version 
[14] S. Nkomo, M. R. Tinsley, and K. Showalter, "Chimera states in populations of nonlocally coupled chemical oscillators," Phys. Rev. Lett. 110, 244102 (2013).

Online Version xi, 22, 24

[15] S. Kádár, T. Amemiya, and K. Showalter, "Reaction mechanism for light sensitivity of the $\mathrm{Ru}(\mathrm{bpy})_{3}^{2+}$ catalyzed Belousov-Zhabotinsky reaction," J. Phys. Chem 101, 8200-8206 (1997). Online Version 22, 23

[16] K. Kalyanasundaram, "Photophysics, photochemistry and solar energy conversion with tris(bipyridyl)ruthenium(ll) and its analagoues." Cood. Chem. Rev. 46, 159-244 (1982).

Online Version 22

[17] R. Toth and A. F. Taylor, "Review of the tris(2,2'-bipyridyl)ruthenium-catalysed BelousovZhabotinsky reaction." Prog. React. Kinet. 31, 59-115 (2006).

Online Version 22, 23, 29

[18] V. K. Vanag, L. Yang, M. Dolnik, A. M. Zhabotinsky, and I. R. Epstein, "Oscillatory cluster patterns in a homogeneous chemical system with global feedback," Nature 406, 389-391 $(2000)$.

Online Version 23

[19] A. F. Taylor, P. Kapetanopoulos, B. J. Whitaker, R. Toth, L. Bull, and M. R. Tinsley, "Phase clustering in globally coupled photochemical oscillators," Eur. Phys. J. Spec. 165, 137-149 (2008).

Online Version 27, 29, 30

[20] T. Ko and G. B. Ermentrout, "Phase-response curves of coupled oscillators," Phys. Rev. E 
79, $016211(2009)$.

Online Version 28

[21] M. A. Schwemmer and T. J. Lewis, "Phase Response Curves in Neuroscience," (Springer Series in Computational Neuroscience, Berlin, 2012).

Online Version 28

[22] R. O. Dror, C. C. Canavier, R. J. Butera, J. W. Clark, and J. H. Byrne, "A mathematical criterion based on phase response curves for stability in a ring of coupled oscillators." Biological Cybernetics 80, 11-23 (1999).

Online Version 28

[23] B. Gutkin, G. B. Ermentrout, and D. A. Reyes, "Phase-response curves give the responses of neurons to transient inputs," J. Neurophysiol. 94, 1623-1635 (2005).

Online Version

[24] A. Granada, R. M. Hennig, B. Ronacher, A. Kramer, and H. Herzel, "Phase response curves elucidating the dynamics of coupled oscillators." Methods Enzymol. 454, 1-27 (2009). Online Version 28

[25] R. Smeal, G. B. Ermentrout, and J. A. White, "Phase-response curves and synchronized neural networks," Philos. Trans. R. Soc. Lond. B. Biol. Sci. 365, 2407-2422 (2010).

Online Version 28

[26] Z. Boulos and B. Rusak, "Circadian phase response curves for dark pulses in the hamster," J. Comp. Physiol 146(4), 411-417 (1982).

Online Version 28 
[27] A. J. Lewy, S. Ahmed, J. M. L. Jackson, and R. Sack, "Melatonin shifts human orcadian rhythms according to a phase-response curve," Chronobiol. Int. 9(5), 380-392 (1992). Online Version 28

[28] M. R. Tinsley, A. F. Taylor, Z. Huang, and K. Showalter, "Complex organizing centers in groups of oscillatory particles," Phys. Chem. Chem. Phys. 13, 17802-17808 (2011). Online Version 30, 34 


\section{Chapter 3}

\section{Chimera and Phase-cluster States}

\subsection{Introduction}

There is considerable interest in the emergence of order and self organization in populations of coupled discrete oscillators. Most studies have focused on the emergence of a collectively uniform dynamical state from a desynchronized state in various systems of globally coupled oscillators [1-16]. In 2002, Kuramoto and Battogtokh reported a highly counter intuitive dynamical state comprised of a subpopulation of oscillators exhibiting synchronous oscillations coexisting with an asynchronous subpoplution [17]. This is counterintuitive in the sense that the oscillators are identical and have the same coupling structure, but they do not exhibit the dynamical same behavior in the two subpopulations. In 2004, Strogatz and Abram named the dynamical state a chimera state [18]. The name chimera has origins in Greek mythology, and is used to describe a fire breathing monster with a lion's head, goat's body, and serpent's tail [18]. A number of numerical models and network topologies have been studied to help understand the dynamics of this exotic state [18-31]. Most of the studies are based on populations of identical oscillators. For at least a decade, the experimental confirmation of the chimera state remained elusive. Experimental 
systems have inherent heterogeneities because the oscillators are not identical, since there is always a distribution of periods. In 2008, Abrams et al. [22] reported a simplified model with identical oscillators supporting chimera states. The model uses a two-group coupling approach to study the dynamics of interacting populations of oscillators [32-34]. Laing [21, 35] reported chimera states in populations of heterogeneous oscillators using this approach. The results provided some insights into the search for chimera states in experimental systems. This chapter provides details of experiments and simulation studies of one of the first experiments showing chimera states. The experiments are based on the photosensitive Belousov-Zhabotinsky chemical oscillators described in the previous chapter.

\subsection{Coupling Model Background}

The study follows the coupling structure in the model proposed by Abrams et al. [22], which divides the population of oscillators into two subpopulations of oscillators that are globally coupled, both within and between the subpopulations. The model is a simplification of the original model of Kuramoto and Battogtokh, which is discussed in detail in the next chapter [17]. The phase model is represented as

$$
\frac{d \theta_{i}^{\sigma}}{d t}=\omega+\sum_{\hat{\sigma}=1}^{2} \frac{K_{\sigma \dot{\sigma}}}{N_{\dot{\sigma}}} \sum_{j=1}^{N_{\hat{\sigma}}} \sin \left(\theta_{j}^{\dot{\sigma}}-\theta_{i}^{\sigma}-\alpha\right),
$$

where $\sigma=1,2, N_{\sigma}$ is the number of members in group $\sigma$, and $K_{\sigma \sigma}$ is the coupling strength [22].

The intragroup coupling $\left(K_{11}=K_{22}\right)$ is greater than the intergroup coupling strength $\left(K_{12}=K_{21}\right)$. Equation (3.1) therefore means that an oscillator experiences strong global coupling to its fellow group members and weaker global coupling to oscillators in the other group. This simulates nonlocal coupling, which is important for the observation of the chimera state $[24,36]$. The oscillators are identical, with natural frequency $\omega$, and are coupled through the sine of their phase 


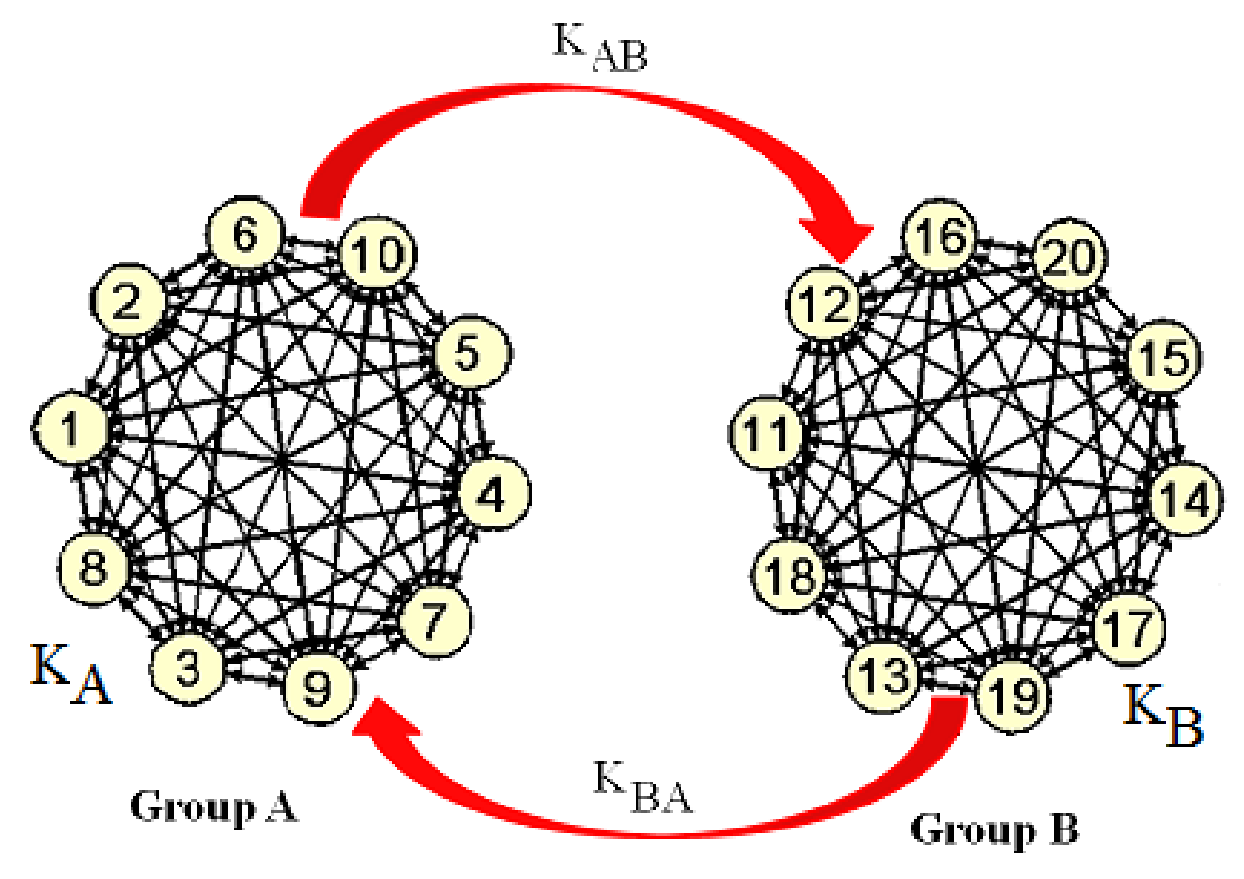

Figure 3.1. Simplified representation of the coupling model with half of the number of oscillators that were used in the experiments.

differences, with an additional phase frustration term $\alpha$, where $0<\alpha<\frac{\pi}{2}[17,21,22,32,37]$. The phase frustration term causes oscillators to synchronize to a frequency different from the average of their natural frequencies $[22,37]$ and plays an important role for the observation of chimera states [36]. Chimera states have been found in heterogeneous populations, and similar features have been found for oscillators with initial phases or natural frequencies chosen from either a Gaussian or Lorentzian distribution $[21,35]$. Sen et al. [24] applied a fixed time-delay $(\tau)$ coupling to a spatial system of identical oscillators and found clustered chimera states with adjacent synchronized antiphase clusters. The delay is associated with the finite time required for a signal to be transmitted [24]. Interesting dynamics of chimera states have been reported in further studies of systems that include delay [23, 26, 28, 38-40]. 


\subsection{Experiments}

Experiments are carried out with 40 discrete chemical oscillators based on the photosensitive BZ reaction $[11,13,41,42]$, in which the $\mathrm{Ru}(\mathrm{bpy})_{3}^{2+}$ catalyst is loaded onto ion-exchange particles that are then immersed in catalyst-free reaction mixture. A detailed description of the experiment set-up is in Section 2.3. The $N$ oscillatory particles are divided into two groups of equal size, A and $\mathrm{B}$, as shown in Figure 3.1, with each member $i$ of group $\sigma$ experiencing the feedback light intensity $P_{i}^{\sigma}$ :

$$
P_{i}^{\sigma}=P_{0}+k_{\sigma} P_{\max }\left(\hat{I}_{\sigma}(t-\tau)-I_{i}(t)\right)+k_{\sigma \sigma^{\prime}} P_{\max }\left(\hat{I}_{\sigma^{\prime}}(t-\tau)-I_{i}(t)\right)
$$

where $\sigma=A, B$ and $\sigma^{\prime}=B, A$. The mean intensity of the oscillators in group $\sigma$ is $\hat{I}_{\sigma}$, and $k_{\sigma}$ and $k_{\sigma \sigma^{\prime}}$ are the intra-group and inter-group coupling coefficients, respectively, with $\left|k_{\sigma \sigma^{\prime}}\right|<k_{\sigma}$. Each member of each group is globally coupled via the mean signal of its own group and the mean signal of the other group, with the intra-group coupling stronger than that of the inter-group coupling. The delay term $\tau[24,27,43]$ plays a role akin to the phase frustration term used in equation (3.1) of the model [22] and in other phase oscillator models of chimera states.

Experiments are carried out as described in Section 2.3.4. An experiment is initiated by applying the intra-group feedback for group $\mathrm{A}$ only $\left(k_{A B}=0, k_{B}=0\right)$, allowing this group to fully synchronize. The group B particles are not coupled and oscillate with approximately random phases. After an initial period, the intra-group feedback for group $\mathrm{B}$, with $k_{B}=k_{A}$, and the intergroup feedback, with $k_{A B}=k_{B A}$, are simultaneously switched on, and the system is monitored for a further 50 minutes. The data are analyzed as described below. 


\subsection{Data Analysis}

\subsubsection{Mean Signal}

The normalized mean intensity of each group was calculated from the normalized individual particle intensities of the group members. A noisy mean signal (Figure 3.2a) represents an asynchronous state. The amplitude grows as the oscillators synchronize (Figure 3.2b) until it is comparable to the individual oscillator amplitude when all of the oscillators are synchronized. Other possible states such as phase-clusters exist between the completely synchronized state and asynchronous state. These are discussed in the results section.

\subsubsection{Order Parameter}

The parameter $r, r(t)=\left|\left\langle\exp ^{i \theta(t)}\right\rangle\right|$, is a measure of phase coherence, averaged over all oscillators in the group at a given time $[17,22]$. For a completely synchronized state $r(t)=1$, while for the completely desynchronized state $r(t) \approx 0[17,22]$.

The ruthenium based BZ system used in the experiments forms phase clusters $[11,13,44,45]$. Figure 3.3, shows the mean signal of a 4-phase-cluster state and the corresponding order parameter. Although these phase clusters are phase locked, the global order parameter is low because the clusters are all out of phase with each other. In experiments that form phase clusters, it is not possible using the global order parameter to distinguish between higher cluster states and asynchronous states. For this reason, the order parameter was not used for the analysis in this study, although it has been used in most of the studies of systems with phase oscillators. 

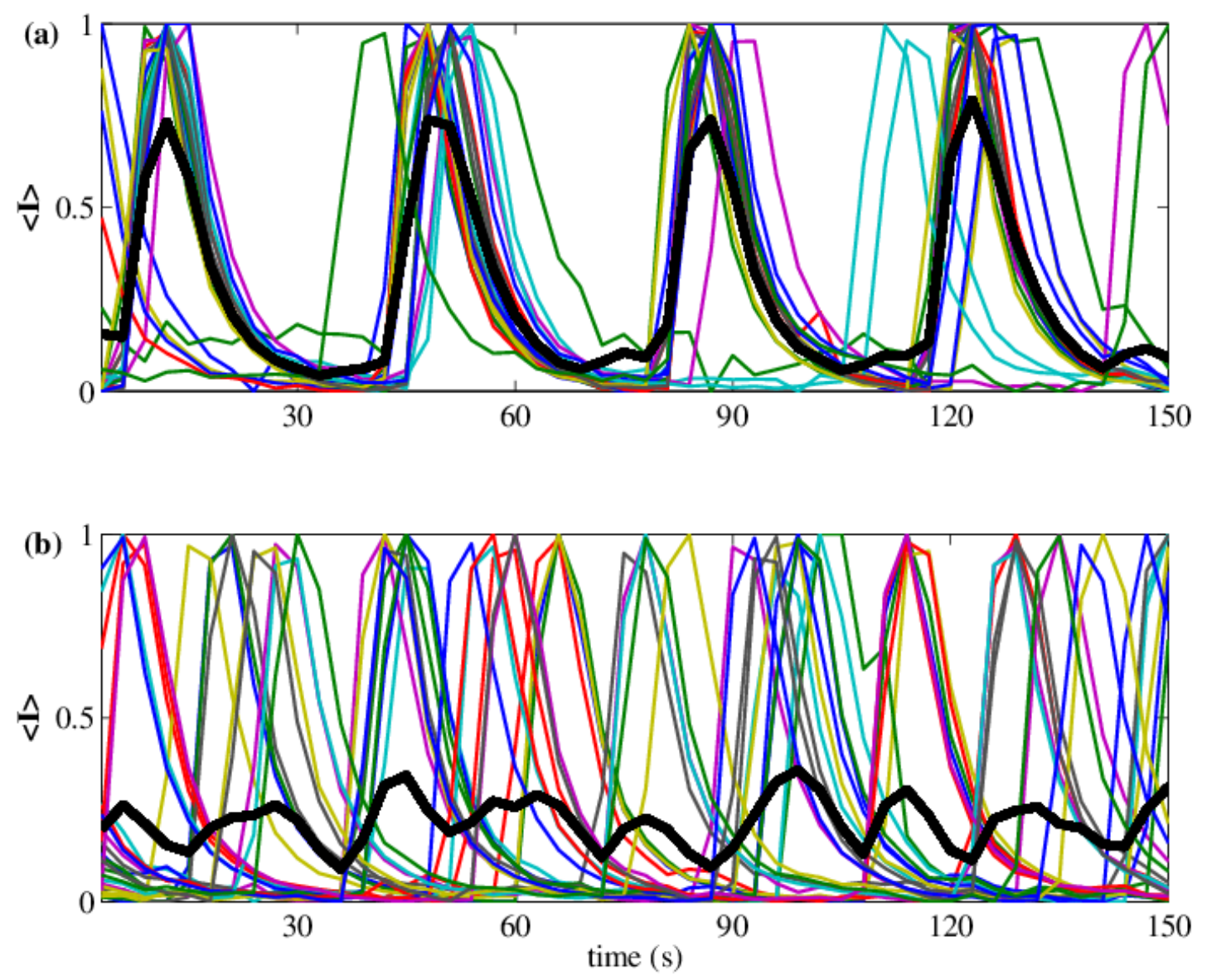

Figure 3.2. Experiments showing variations of individual intensities of (a) asynchronous oscillators, (b) synchronizing oscillators, and the mean signal (bold black) with time. 

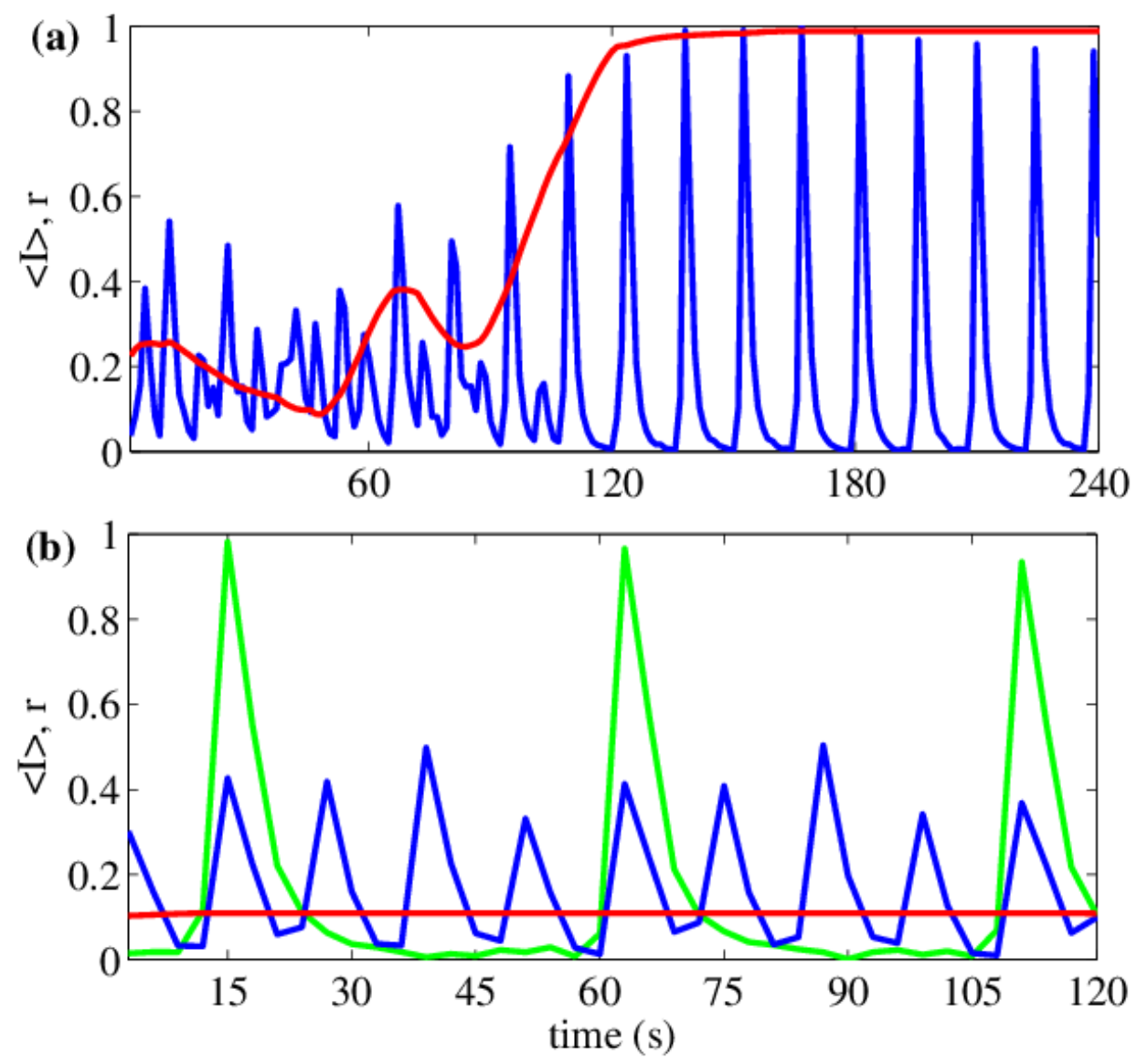

Figure 3.3. (a) Normalized mean intensity (blue) and the global order order parameter $r$ (red).

(b) Normalized mean intensity (blue), normalized individual oscillator intensity (green), and the global order order parameter $r$ (red). 


\subsubsection{Period and Phase Determination}

The times of occurrence of the maximum intensities (peak times) were determined using a MATLAB algorthim. The period in seconds is given by multiplying the frame rate by the time interval between adjacent maxima, as represented by the equation

$$
T=\left(t_{p_{n+1}}-t_{p_{n}}\right) \times \text { frame rate }
$$

where $t_{p_{n}}$ and $t_{p_{n+1}}$ are the times of the $n^{\text {th }}$ and $(n+1)^{t h}$ peaks, respectively.

The phases, $\varphi$, are claculated by linear interpolation between adjacent maxima:

$$
\varphi_{i}=2 \pi\left(t t-t_{p_{n}}\right) / T
$$

where $t_{p_{n}} \leq t t \leq t_{p_{n+1}}$.

\subsection{Experiment Results}

The coupling scheme gives rise to several different types of synchronization behavior in our experiments. In all cases, group A remains fully synchronized, while group B exhibits four basic states: fully synchronized, n-cluster, the unsynchronized chimera, and semi-synchronized. We label these states $1-1,1-\mathrm{n}(\mathrm{n}=2,3,4, \ldots), 1-\mathrm{c}$ and $1-\mathrm{s}$, respectively, where the first number represents the state of group A and the second number (or letter) represents the state of group B.

During full synchronization, the 1-1 state, members of group B synchronize both in frequency and phase [43], typically with a constant phase difference relative to the synchronized members of group A. Figure 3.4a shows a time series of the mean oscillator intensity in groups A and B, and the inset shows a snapshot of the phase of each oscillator in the two groups. An example of a two-cluster state [46] is shown in Figure 3.4b, where the different occupancies of the two clusters 

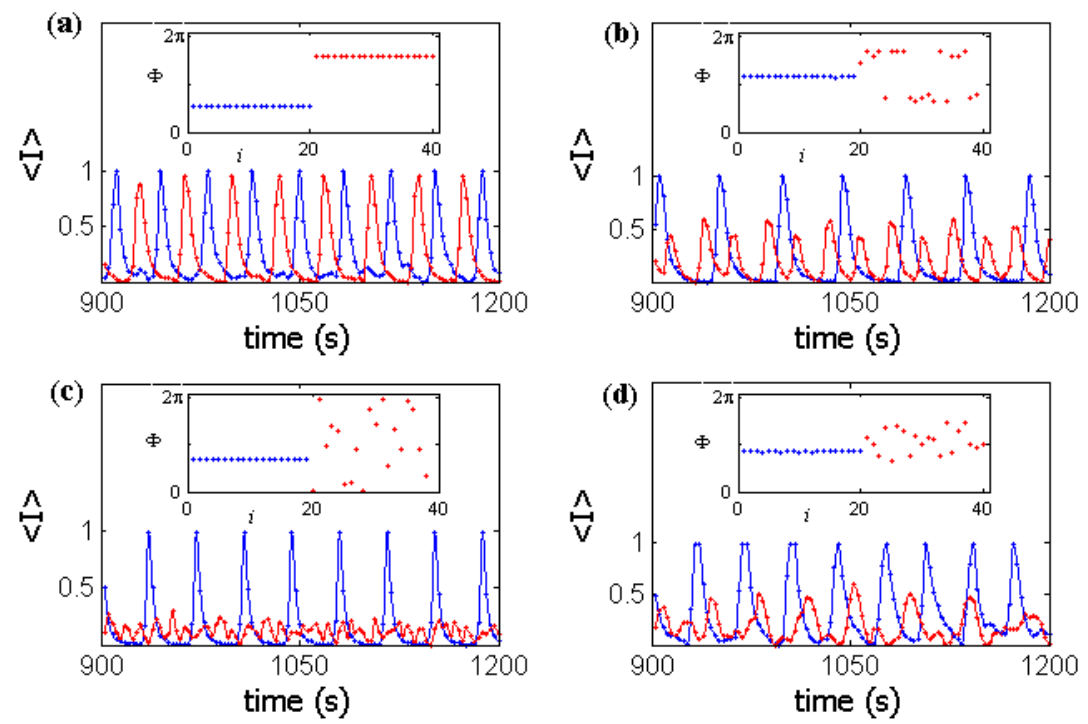

Figure 3.4. Normalized mean intensity of group A (blue) and group B (red) as a function of time, and (inset) snapshot of the phases of oscillators 1-20 in group A and 21-40 in group B. (a) Synchronized out-of-phase 1-1 state, $k_{A}=3.0, k_{A B}=-1.8$; (b) synchronized phase cluster 1-2 state, $k_{A}=4.0, k_{A B}=-1.0 ;$ (c) chimera 1-c state, $k_{A}=3.0, k_{A B}=-0.2$; and $(\mathrm{d})$ semisynchronized 1-s state, $k_{A}=2.0, k_{A B}=-1.0$. Time series curves show fits to data points (o). The phase $0-2 \pi$ of an individual oscillator (inset) is assigned by linear interpolation between adjacent maxima. In all of the experiments $\tau=30 \mathrm{~s}$.

are visible in the phase snapshot. We see a wide range of occupancies in the two-cluster state as well as in higher order 1-3 and 1-4 states in our experiments.

In the 1-c chimera state, the members of group B remain unsynchronized for the entire duration of the experiment. The mean intensity for group B is a small-amplitude, noisy signal, as shown in Figure 3.4c. At any moment, the phases of the oscillators are spread almost evenly between 0 and $2 \pi$ (see inset). In addition to chimera states, we also observe semi-synchronized states, which involve higher and lower frequency oscillators intermittently aligning, resulting in occasional mean 
intensities with irregular large amplitudes, Figure 3.4d. An example of transient partial alignment leading to larger values in mean amplitude is shown in the phase snapshot. The behaviors shown in Figure 3.4 are found at different intra- and inter-group coupling strengths, and the various behavior regimes as a function of $k_{A}=k_{B}$ and $k_{A B}=k_{B A}$ are shown in Figure 3.5a. The synchronized 1-1 state is in-phase or out-of-phase for $k_{A B}>0$ or $k_{A B}<0$, respectively.

The 1-c chimera region shown in Figure 3.5a occurs primarily with the inter-group coupling strength $k_{A B}<0$, although there is a small region of 1 -c behavior for $k_{A B}>0$. The chimera for negative inter-group coupling strengths was surprising in view of the model of Abrams et al. [22], which employs only positive coupling, and we therefore investigated the underlying coupling mechanism. As discussed in Section 2.4 in Chapter 2, curves for perturbations involving both increases and decreases in light intensity give rise to positive phase responses. The result means that both positive and negative values of $k_{A B}$ give rise to positive inter-group coupling, consistent with the theoretical model [22].

\subsection{Simulations}

\subsubsection{Model}

The experimental system was modeled with a two-variable model $[11,13]$ for the photosensitive BZ particle system $[11,13,44,45]$, where $d X_{i} / d t=f\left(X_{i}, Z_{i}, q_{i}\right)+\phi_{i}^{\sigma}, d Z_{i} / d t=g\left(X_{i}, Z_{i}, q_{i}\right)+2 \phi_{i}^{\sigma}$ describes the chemistry of each oscillator $i$ in group $\sigma$ (see Section 2.2.4). The terms $f$ and $g$ give the non-photochemical components of the BZ reaction, and $X_{i}, Z_{i}$, and $q_{i}$ are $\left[\mathrm{HBrO}_{2}\right]$, $\left[\mathrm{Ru}(\mathrm{bpy})^{3+}\right]$, and the stoichiometric coefficient, respectively, associated with the $i^{\text {th }}$ oscillator. 

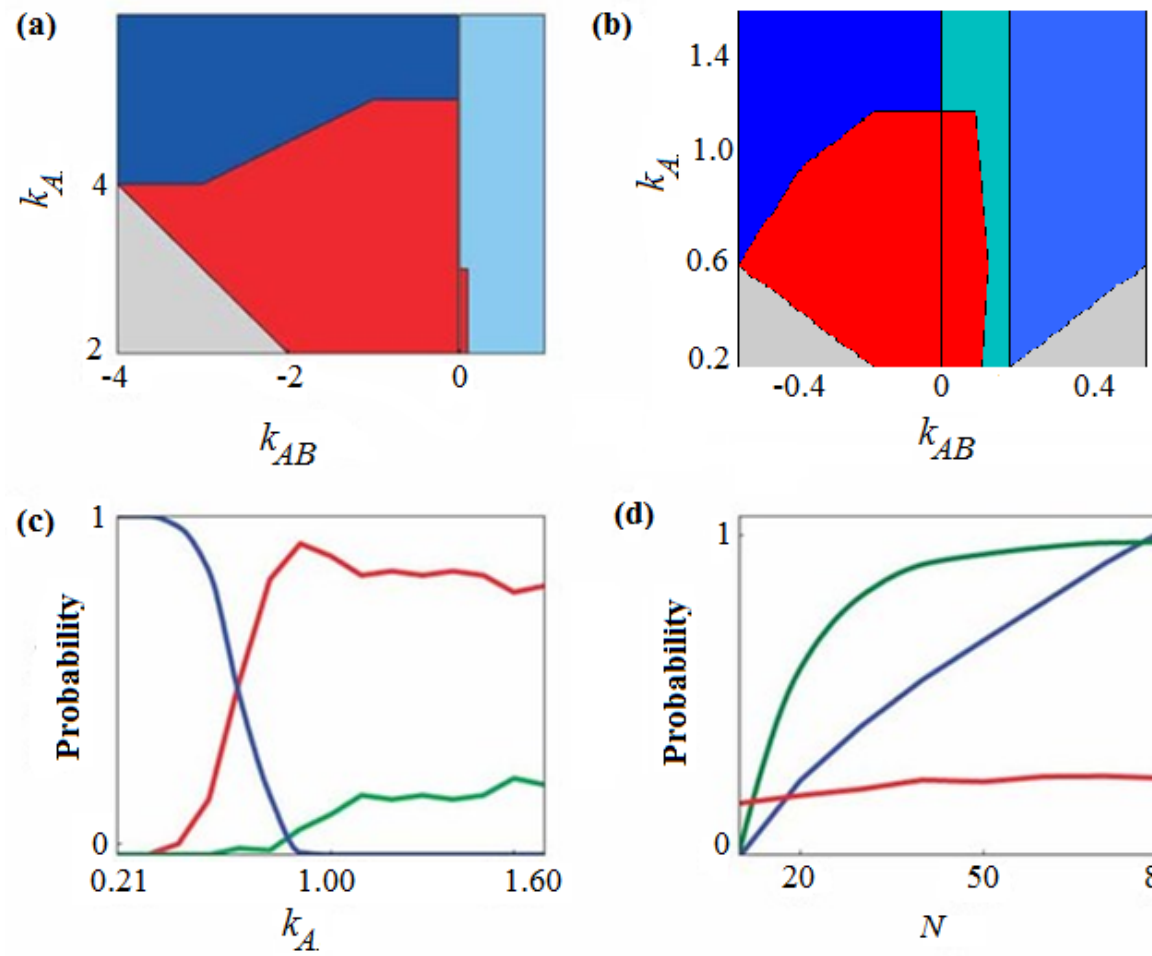

(d)

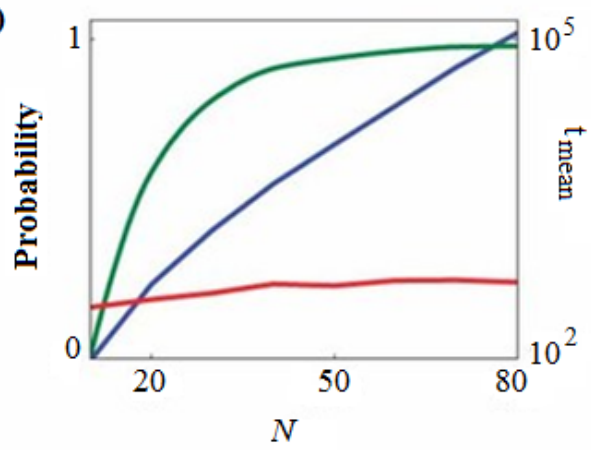

Figure 3.5. Dependence of the chimera and cluster states on parameters. Regions of 1-c chimera behavior (red), 1-1 in-phase (light blue), and 1-1 out-of-phase (dark blue) synchronization observed in experiment as a function of coupling strengths $k_{A}$ and $k_{A B}$ : (a) Experiment results, (b) Simulation results. (c) Probability of 1-c (blue), 1-1 (red), and 1-2 (green) states as a function of $k_{A}$ from simulations of the homogeneous frequency model system, with $N=80$. Continuity methods yield stable states in the following regions: 1-1 state stable for all values of $k_{A}, 1-2$ state stable for $0.23<k_{A}<1.6$, 1 -c state stable for $0.21<k_{A}<0.8$. (d) Probability of stable chimera state (green) for homogeneous frequency system as a function of number of oscillators $N$. Logarithm of mean time to collapse to synchronized state for homogeneous (red) and heterogeneous (blue) frequency oscillator populations. Probabilities in (c) and (d) are based on 400 simulations for each abscissa value using random initial phases for group $\mathrm{B}$; conditions: $\tau=36, k_{A}=0.6, k_{A B}=0.12$, except where otherwise indicated. 
The photo-excitatory feedback to oscillator $i$ in group $\sigma$ is $\phi_{i}^{\sigma}$, calculated according to

$$
\phi_{i}^{\sigma}=\phi_{0}+k_{\sigma} \phi_{\max }\left(\hat{Z}_{\sigma}(t-\tau)-Z_{i}(t)\right)+k_{\sigma \sigma^{\prime}} \phi_{\max }\left(\hat{Z}_{\sigma^{\prime}}(t-\tau)-Z_{i}(t)\right)
$$

with other terms as in Equation (3.5). Simulations are carried out with up to 80 particles (40 per

group) for both homogeneous (period $=41.0)$ and heterogeneous (period $=41.0 \pm 2.1$ ) oscillator systems. The heterogeneous system has an approximately normal distribution in period.

A simulation is initiated by synchronizing the oscillators in group A by applying intra-group feedback to that group only. Intra-group feedback is then applied to the oscillators in group B at $\mathrm{t}=200$, with $k_{B}=k_{A}$, where the phase of each oscillator is selected from a random distribution. Inter-group feedback is also applied at this time, with $k_{A B}=k_{B A}$. Simulations are typically carried out until $t=100,000$, and the state of the system is then recorded. The mean amplitude of the chimera is a noisy, flat signal, allowing an empirical threshold in the mean signal amplitude to be established and computationally employed to test for the existence of chimera states and to distinguish the chimera from other states such as semi-synchronized states. A mean signal threshold of 0.35 was found to be satisfactory for detecting the collapse of a chimera state and distinguishing it from other states.

\subsubsection{Simulation Results}

The model simulations generate behavior in qualitative agreement with that exhibited by the experimental system, namely, the 1-1, 1-n, 1-c and 1-s states. Standard continuation methods were used to determine whether or not a state is stable in a given parameter region and whether other stable states coexist. The location of these states in the $k_{A^{-}} k_{A B}$ phase diagram, Figure $3.5 \mathrm{~b}$, is similar to the experimental behavior shown in Figure 3.5a, with a small region of chimera behavior observed for $k_{A B}>0$ and a much larger region observed for $k_{A B}<0$. 
The probability of the homogeneous frequency system residing in the 1-c, 1-1, or 1-2 state as a function of $k_{A}$ is shown in Figure 3.5c. At low values of $k_{A}$, the system predominantly finds the chimera state, with the 1-1 and 1-2 states increasingly prevalent at higher values of $k_{A}$. Nonstationary (breathing) chimera states were also found in certain parameter regimes, similar to those reported by Abrams et al. [22].

No semi-synchronized behavior is found in the homogeneous frequency system, since when partial phase alignment occurs, the system quickly relaxes to a 1-1 or 1-n state. In the simulations for Figure 3.5c, for example, the system rapidly evolved to a 1-1 or 1-2 state when the mean signal of group B exceeded a threshold of 0.35 , indicating partial alignment.

Simulations were also carried out to determine the effects of group size and frequency heterogeneity on the probability of the system residing in the 1-c chimera state. For the homogeneous frequency system, the probability of the 1-c state increases with increasing group size, as shown in Figure 3.5d, where the 1-1 state is exhibited when the chimera state is not found. Also shown in Figure 3.5d is the lifetime of asynchronous states that collapsed to synchronized states. The relatively short lifetime of these collapsing states is almost constant, and they apparently arise from initial conditions unfavorable to finding the chimera state. In contrast, the system with a distribution of oscillatory frequencies exhibits chimera lifetimes that increase approximately exponentially with increasing system size, Figure 3.5d. No asymptotically stable chimera is observed in this system for this level of period heterogeneity $(41.0 \pm 2.1)$; however, extremely long-lived 1-c states are observed for larger system sizes $\left(>10^{4}\right.$ periods for $\left.N=70\right)$.

The small-amplitude mean signal of the chimera in the homogeneous frequency system is illustrated in Figure 3.6a, as well as the large-amplitude mean signal of the system when the initial conditions lead quickly to a synchronized 1-1 state. Figure 3.6b shows a long-lived chimera in the heterogeneous frequency system, with a noisy small-amplitude mean signal, along with the collapse 
of a chimera to a synchronized 1-1 state for the same conditions but with a different initial phase distribution. Figure 3.6c shows a long-lived chimera state along with a chimera collapsing to a synchronized 1:1 state in two experiments with the same conditions.

The effects of period heterogeneity on the chimera lifetime can be seen in a plot of mean lifetime as a function of the standard deviation of the period distribution, shown in Figure 3.6d. The mean lifetime dramatically increases at low heterogeneity of the oscillator population. The simulations indicate that there is a small but finite range of very low period heterogeneity in which the chimera states are asymptotically stable.

Our studies of coupled chemical oscillators show that the heterogeneous frequency system, corresponding to the experiments and associated simulations, exhibits chimera, cluster and fully synchronized states. The chimera lifetime grows approximately exponentially with system size (Figure 3.5d), and we find evidence of asymptotically stable chimera behavior at very low levels of heterogeneity (Fig. 3.6d). Interestingly, simulations of the homogeneous frequency system indicate that asymptotically stable chimera states occur and increase in occurrence with increasing system size (Figure 3.5d.) This is in contrast with recent studies by Wolfrumand and Omel'chenko [47], which demonstrate that the chimera state is transient for finite system sizes for identical phase oscillators. This suggests that the relaxation oscillations of the BZ system lend stability to the chimera state, as this is the primary feature that differs between these systems. We note that extremely long-lived chimera states in our simulations do not provide unequivocal evidence for asymptotic stability of these states.

The chimera behavior found in our experiments and corresponding simulations of the heterogeneous frequency system is similar in many respects to the behavior found by Wolfrumand and Omel'chenko [47] in a homogeneous phase oscillator model. These authors point out that above small system sizes, the chimera state becomes very long- lived, and the behavior is much like that 

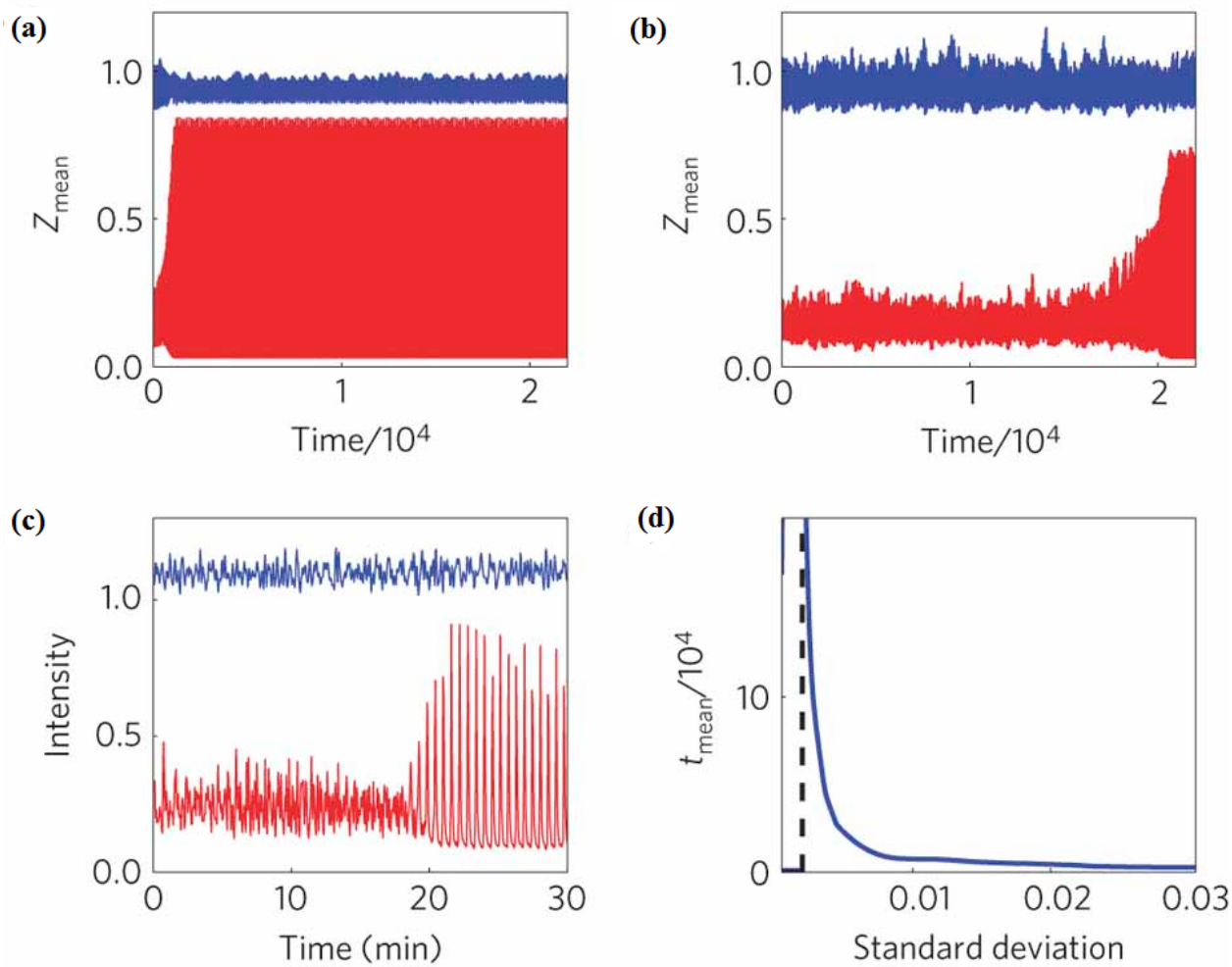

Figure 3.6. Long-lived and transient chimera states in simulations and experiments. (a) Time series for chimera state (blue) and transient to a 1-1 state (red) in the homogeneous frequency model, with $N=60$. (b) Chimera state (blue) and collapse to synchronization (red) in the heterogeneous frequency model, with $N=60$. (c) Sustained chimera state (blue) and example of a collapse to synchronization (red) in experiment, with $k_{A}=3.0, k_{A B}=-1.0$ in both cases. (d) Time to collapse in the heterogeneous frequency model as a function of standard deviation of the parameter $q$ determining the period, with $N=40$. The dashed line shows the approximate location of the asymptote of the divergent chimera lifetime. Other parameters for (a), (b), and (d) as in Figure 3.5. The time series shown in (a), (b), and (c) are the mean signal for group B, with the blue time series offset for illustration purposes. 


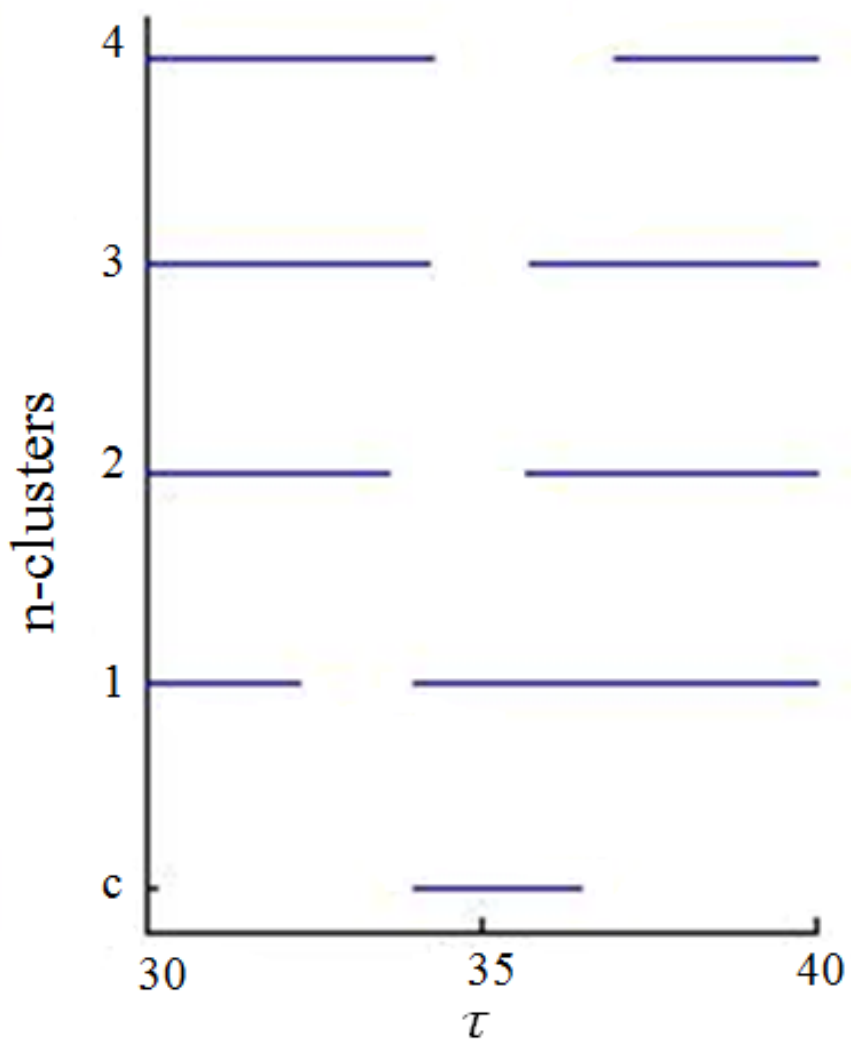

Figure 3.7. Continuity results showing stable regions for the 1-1, 1-c and 1-n states vs time delay $(\tau)$.

observed in the thermodynamic limit, $N \rightarrow \infty$.

\subsection{Multistability Experiments}

Continuity methods in simulations demonstrate that the chimera is embedded in a region of multistability, with the 1-1 state occurring over the entire range of $k_{A}$ and small regions of higherorder 1-2, 1-3 and 1-4 cluster states also occurring as shown in Figure 3.7.

Experiments to demonstrate multistability of states were carried out using perturbation methods. Experiments were initiated as before, with oscillators in group A synchronized, while those in 


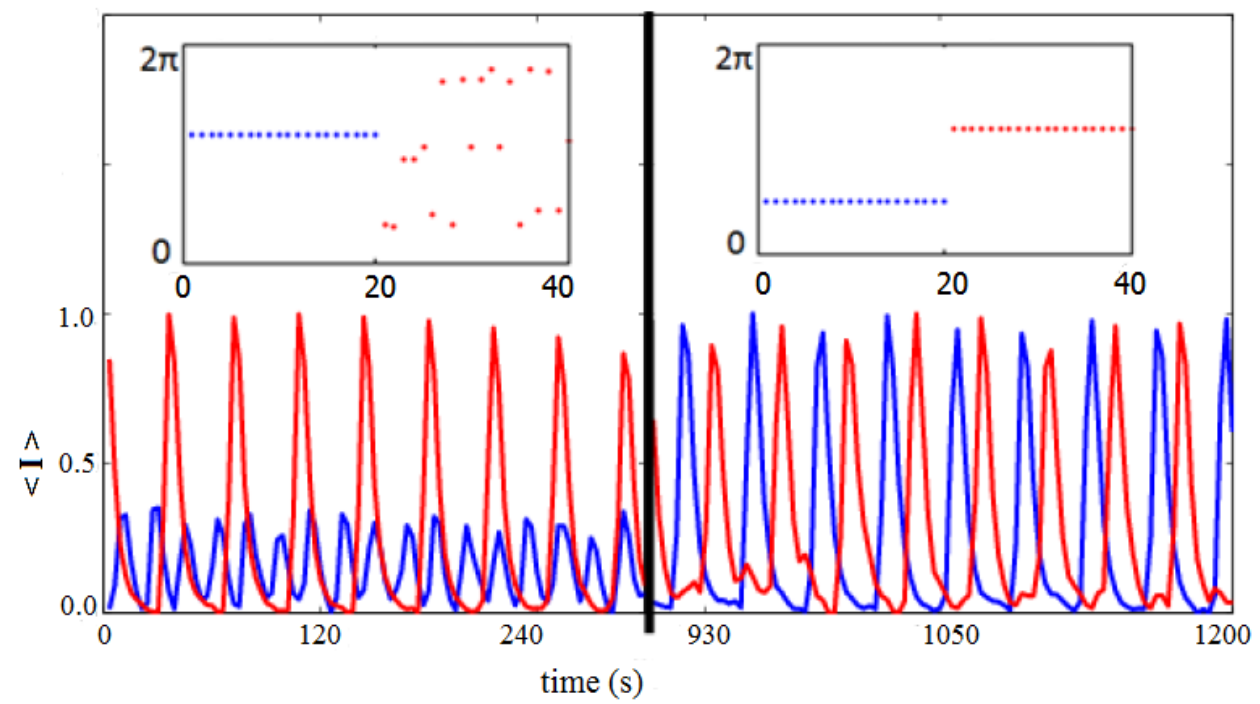

Figure 3.8. Experiment showing stable 1-3 culster state before perturbation and 1-1 state after perturbation.

group B had a random phase distribution. The coupling was introduced for 20 min. The sytem was perturbed by turning off the coupling in group B for 5 min. The coupling scheme was reintroduced for a further 20 min. As shown in Figure 3.8, the system initially evolved to a 1-3 state, but after pertubation a 1-1 state was observed. Other cluster states, 1-2 and 1-4, were observed with the same conditions and parameters as the chimera state.

\subsection{Summary}

In conclusion, the chimera state in populations of coupled chemical oscillators is made up of subpopulations that display coherent and incoherent oscillations but are otherwise exactly the same. The chimera behavior is not the result of frequency heterogeneity in our system, since, in fact, frequency heterogeneity reduces the lifetime and hence the likelihood of finding the state (Figure 3.6d). Chemical oscillators are stiff, relaxation oscillators, which may give rise to important differ- 
ences in their chimera behavior from the more idealized theoretical models. The semi-synchronized state in this system remains to be fully characterized, but we believe that it may be the result of transient phase clusters (switchers) that are known to occur in related globally-coupled BZ systems $[11,13,45]$. Demonstrations of chimera states in experimental systems [48-50] motivated the study discussed in the next chapter of the original Kuramoto-like coupling structure of chemical oscillators.

The chimera's very existence is a surprise, and we note that this peculiar dynamical behavior could be of importance in certain biological and physical systems. Many birds as well as dolphins sleep with "one eye open" in the sense that one hemisphere of the brain is "in sync" while the other is asynchronous $[22,51]$. In addition, the simultaneous appearance of laminar and turbulent regions in Couette flow [52] represents the coexistence of coherent and incoherent behavior. 


\section{References}

[1] S. H. Strogatz, "From Kuramoto to Crawford: Exploring the onset of synchronization in populations of coupled oscillators," Physica D 143, 1-20 (2000).

Online Version 40

[2] M. Choi, H. Kim, D. Kim, and H. Hong, "Synchronization in a system of globally coupled oscillators with time delay," Phys. Rev. E 61, 371-81 (2000).

Online Version

[3] R. E. Mirollo and S. H. Strogatz, "Synchronization of pulse-coupled biological oscillators," SIAM J. Appl. Math. 50, 1645-1662 (1990).

Online Version

[4] J. G. Restrepo, E. Ott, and B. R. Hunt, "Synchronization in large directed networks of coupled phase oscillators." Chaos 16, 015107 (2006).

Online Version

[5] A. S. Pikovsky, M. G. Rosenblum, and J. Kurths, "Synchronization in a population of globally coupled chaotic oscillators," Europhys. Lett. 34, 165-170 (2007).

Online Version

[6] H. Sakaguchi, "Stochastic synchronization in globally coupled phase oscillators." Phys. Rev. E 66, 056129 (2002).

Online Version

[7] B. C. Daniels, "Synchronization of globally coupled nonlinear oscillators: The rich behavior of the Kuramoto model," Analysis 1-54 (2005).

Online Version 
[8] J. Rougemont and F. Naef, "Collective synchronization in populations of globally coupled phase oscillators with drifting frequencies." Phys. Rev. E 73, 011104 (2006). Online Version

[9] W. Wang, I. Z. Kiss, and J. L. Hudson, "Experiments on arrays of globally coupled chaotic electrochemical oscillators: Synchronization and clustering." Chaos 10, 248-256 (2000).

Online Version

[10] R. Toth, A. F. Taylor, and M. R. Tinsley, "Collective behavior of a population of chemically coupled oscillators." J. Phys. Chem. B 110, 10170-10176 (2006).

Online Version

[11] A. F. Taylor, P. Kapetanopoulos, B. J. Whitaker, R. Toth, L. Bull, and M. R. Tinsley, "Clusters and switchers in globally coupled photochemical oscillators." Phys. Rev. Lett. 100, 214101 (2008).

Online Version 43, 44, 49, 57

[12] I. Kiss, M. Quigg, S. C. Chun, H. Kori, and J. Hudson, "Characterization of synchronization in interacting groups of oscillators: Application to seizures." Biophysical 94, 1121-1130. (2008). Online Version

[13] A. F. Taylor, M. R. Tinsley, F. Wang, and K. Showalter, "Phase clusters in large populations of chemical oscillators," Angew. Chem. Int. Ed. 50, 10161-10164 (2011). Online Version 43, 44, 49, 57

[14] L. M. Pecora, "Synchronization of oscillators in complex networks," Pramana 70, 1175-1198 (2008).

Online Version 
[15] M. Chavez, D.-U. Hwang, A. Amann, H. G. E. Hentschel, and S. Boccaletti, "Synchronization is enhanced in weighted complex networks." Phys. Rev. Lett. 94, 218701 (2005).

Online Version

[16] M. Brede, "Local vs global synchronization in networks of non-identical Kuramoto oscillators." Eur. Phys. J. B 62, 87-94 (2008).

Online Version 40

[17] Y. Kuramoto and D. Battogtokh, "Coexistence of coherence and incoherence in nonlocally coupled phase oscillators," Nonlinear Phenom. in Complex Syst. 5, 380-385 (2002).

Online Version 40, 41, 42, 44

[18] D. M. Abrams and S. Strogatz, "Chimera states for coupled oscillators," Phys. Rev. Lett. 93, $174102(2004)$

Online Version 40

[19] E. A. Martens, "Chimeras in a network of three oscillator populations with varying network topology." Chaos 20, 043122 (2010).

Online Version

[20] C. R. Laing, K. Rajendran, and L. G. Kevrekidis, "Chimeras in random non-complete networks of phase oscillators." Chaos 22, 013132 (2012).

Online Version

[21] C. R. Laing, "Chimera states in heterogeneous networks." Chaos 19, 013113 (2009).

Online Version 41, 42

[22] D. M. Abrams, R. Mirollo, S. H. Strogatz, and D. A. Wiley, "Solvable model for chimera 
states of coupled oscillators," Phys. Rev. Lett. 101, 084103 (2008).

Online Version 41, 42, 43, 44, 49, 52, 57

[23] O. E. Omel'chenko, Y. L. Maistrenko, and P. A. Tass, "Chimera states: The natural link between coherence and incoherence," Phys. Rev. Lett. 100, 044105 (2008).

Online Version 42

[24] G. C. Sethia, A. Sen, and F. M. Atay, "Clustered chimera states in delay-coupled oscillator systems." Phys. Rev. Lett. 100, 144102 (2008).

Online Version 41, 42, 43

[25] D. M. Abrams and S. H. Strogatz, "Chimera states in a ring of nonlocally coupled oscillators," Int. J. Bifurcation Chaos 16, 21-37 (2005).

Online Version

[26] J. H. Sheeba, V. K. Chandrasekar, and M. Lakshmanan, "Globally clustered chimera states in delay-coupled populations." Phys. Rev. E 79, 055203 (2009).

Online Version 42

[27] M. Shanahan, "Metastable chimera states in community-structured oscillator networks." Chaos 20, 013108 (2010).

Online Version 43

[28] R. Ma, J. Wang, and Z. Liu, "Robust features of chimera states and the implementation of alternating chimera states," Europhys. Lett. 91, 40006 (2010).

Online Version 42

[29] M. Wildie and M. Shanahan, "Metastability and chimera states in modular delay and pulse- 
coupled oscillator networks." Chaos 22, 043131 (2012).

Online Version

[30] O. E. Omel'chenko, M. Wolfrum, and Y. L. Maistrenko, "Chimera states as chaotic spatiotemporal patterns." Phys. Rev. E 81, 065201 (2010).

Online Version

[31] I. Omelchenko, Y. Maistrenko, P. Hövel, and E. Schöll, "Loss of coherence in dynamical networks: Spatial chaos and chimera states." Phys. Rev. Lett. 106, 234102 (2011).

Online Version 40

[32] E. Montbrió, J. Kurths, and B. Blasius, "Synchronization of two interacting populations of oscillators," Phys. Rev. E 70, 056125 (2004).

Online Version 41, 42

[33] E. Barreto, B. Hunt, E. Ott, and P. So, "Synchronization in networks of networks: The onset of coherent collective behavior in systems of interacting populations of heterogeneous oscillators." Phys. Rev. E 77, 036107 (2008).

Online Version

[34] P. So, B. C. Cotton, and E. Barreto, "Synchronization in interacting populations of heterogeneous oscillators with time-varying coupling," Chaos 18, 037114 (2008).

Online Version 41

[35] C. R. Laing, "The dynamics of chimera states in heterogeneous Kuramoto networks," Physica D 238, $1569-1588(2009)$.

Online Version 41, 42 
[36] O. E. Omel'chenko, M. Wolfrum, S. Yanchuk, Y. L. Maistrenko, and O. Sudakov, "Stationary patterns of coherence and incoherence in two-dimensional arrays of non-locally-coupled phase oscillators," Phys. Rev. E 85, 036210 (2012).

Online Version 41, 42

[37] H. Sakaguchi and Y. Kuramoto, "A soluble active rotater model showing phase transitions via mutual entertainment," Prog. Theor. Phys. 76, 576-581 (1986).

Online Version 42

[38] J. H. Sheeba, V. K. Chandrasekar, and M. Lakshmanan, "Chimera and globally clustered chimera: Impact of time delay." Phys. Rev. E 81, 046203 (2010).

Online Version 42

[39] A. Sen, R. Dodla, G. L. Johnston, and G. C. Sethia, Amplitude death, synchrony, and chimera states in delay coupled limit cycle oscillators (Springer, Berlin, 2010).

Online Version

[40] O. E. Omel'chenko, Y. L. Maistrenko, and P. A. Tass, "Chimera states induced by spatially modulated delayed feedback." Phys. Rev. E 82, 066201 (2010).

Online Version 42

[41] A. Zaikin and A. M. Zhabotinsky, "Concentration wave propagation in two-dimensional liquidphase self-oscillating system." Nature 225, 535-537 (1970).

Online Version 43

[42] L. Kuhnert, "A new optical photochemical memory device in a light-sensitive chemical active medium," Nature 319, 393-394 (1986).

Online Version 43 
[43] A. Pikovsky, M. Rosenblum, and J. Kurths, Synchronization: A Universal Concept in Nonlinear Sciences (Cambridge University Press, Cambridge, 2003). 43, 47

[44] L. Yang, M. Dolnik, A. M. Zhabotinsky, and I. R. Epstein, "Oscillatory clusters in a model of the photosensitive Belousov-Zhabotinsky reaction system with global feedback," Phys. Rev. E 62, 6414-6420 (2000).

Online Version 44, 49

[45] A. F. Taylor, P. Kapetanopoulos, B. J. Whitaker, R. Toth, L. Bull, and M. R. Tinsley, "Phase clustering in globally coupled photochemical oscillators," Eur. Phys. J. Spec. 165, 137-149 (2008).

Online Version 44, 49, 57

[46] D. Golomb, D. Hansel, B. Shraiman, and H. Sompolinsky, "Clustering in globally coupled phase oscillators," Phys. Rev. A 45, 3516-3530 (1992).

Online Version 47

[47] M. Wolfrum and O. E. Omel'chenko, "Chimera states are chaotic transients." Phys. Rev. E 84, 015201 (2011).

Online Version 53

[48] M. R. Tinsley, S. Nkomo, and K. Showalter, "Chimera and phase-cluster states in populations of coupled chemical oscillators," Nature Physics 8, 662-665 (2012).

Online Version 57

[49] A. M. Hagerstrom, T. E. Murphy, R. Roy, P. Hövel, I. Omelchenko, and E. Schöll, "Experimental observation of chimeras in coupled-map lattices," Nature Physics 8, 658-661 (2012). Online Version 
[50] E. A. Martens, S. Thutupalli, A. Fourrière, and O. Hallatschek, "Chimera states in mechanical oscillator networks." PNAS. 110, 10563-7 (2013).

Online Version 57

[51] N. C. Rattenborg, C. J. Amlaner, and S. L. Lima, "Behavioral, neurophysiological and evolutionary perspectives on unihemispheric sleep." Neurosci. Biobehav. Rev. 24, 817-842 (2000). Online Version 57

[52] D. Barkley and L. S. Tuckerman, "Computational study of turbulent laminar patterns in couette flow," Phys. Rev. Lett. 94, 014502 (2005).

Online Version 57 


\section{Chapter 4}

\section{Chimera States in Populations of}

\section{Nonlocally Coupled Chemical Oscillators}

\subsection{Introduction}

The interest in chimera states continues to grow since their discovery in 2002 by Kuramoto and Battogtokh [1]. Kuramoto et al. [1] used a nonlocal coupling scheme in a system of oscillators in a ring configuration. Nonlocal coupling schemes, where each oscillator is coupled to a range of nearby oscillators, lie between the extremes of global and local coupling [1-8]. For example, nonlocal coupling is found in neuronal networks. Kuramoto et al. [1] used an exponential coupling function to achieve nonlocal coupling of the oscillators. Abrams et al. [3, 4] used a cosine kernel to find an exact solution for a chimera state on a ring of identical phase oscillators. In 2008, Sethia et al. [5] included delay to a system of phase oscillators and discovered clustered chimera states. Chimera states have also been reported in $2 \mathrm{D}$ systems. In the $2 \mathrm{D}$ system, the chimera state can take the form of a synchronized spiral wave rotating around a region of unsynchronized oscillators [2]. Recently, Panaggio et al. [9] reported chimera states on a flat torus, including an 
asymmetric chimera state and other forms of chimera states, which were described as 'spot' and 'stripe' chimeras. Numerical and experimental studies have demonstrated the robustness of chimera states. Examples of experimental systems where chimera states have been found include coupledmap lattices (CML) studied by Hagerstrom et al. [10], populations of chemical oscillators studied

by Tinsley et al. [11], and populations of mechanical oscillators studied by Martins et al. [12]. A detailed descripition of a two-group coupling scheme in populations of chemical oscillators is found in Chapter 3.

This chapter describes synchronization studies of a population of coupled chemical oscillators in a ring configuration. Oscillators are coupled in a Kuramoto-like, scheme with nearest neighbors experiencing the strongest coupling, which decreases exponentially with distance, as shown in Figure 4.1. Periodic boundary conditions are used to create the ring configuration. The dynamical behavior of heterogeneous oscillators in both experiments and simulations is discussed. In addition to the classical chimera state and phase-cluster chimera states, phase-wave chimera states and complex coexistence of these states are dicussed.

\subsection{Nonlocal Coupling Scheme}

This study explores the dynamics of nonlocally coupled chemical oscillators arranged in a ring configuration. The coupling relation is governed by an exponential decay function given by

$$
K=K^{\prime} \exp [-\kappa|(\rho-j)|]
$$

where $K^{\prime}$ and $\kappa$ are constants that govern the effective coupling strength and range of each oscillator. The coupling radius is the number of neighbors on each side of oscillator $j$ that are coupled to it. Equation (4.1) shows that coupling strength decreases with oscillator proximity. 


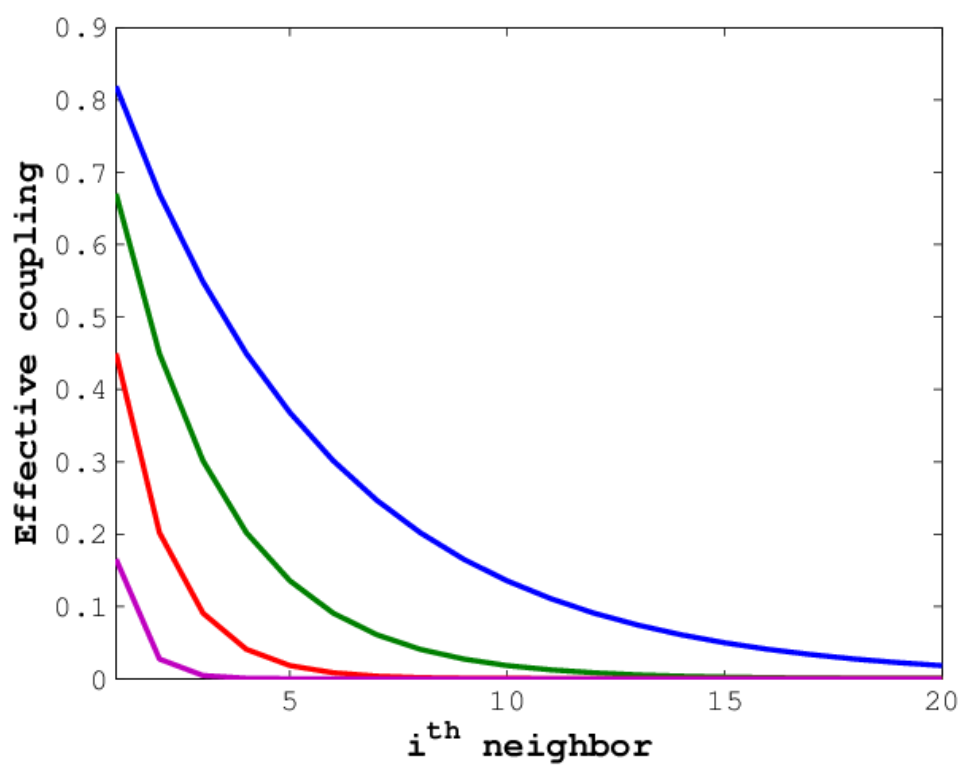

Figure 4.1. Coupling strength vs $i^{\text {th }}$ neighbor for different values of $\kappa$ with $\mathrm{K}^{\prime}=1 ; \kappa=0.2$ (blue), $\kappa=0.4$ (green), $\kappa=0.6$ (red), and $\kappa=2.0$ (purple).

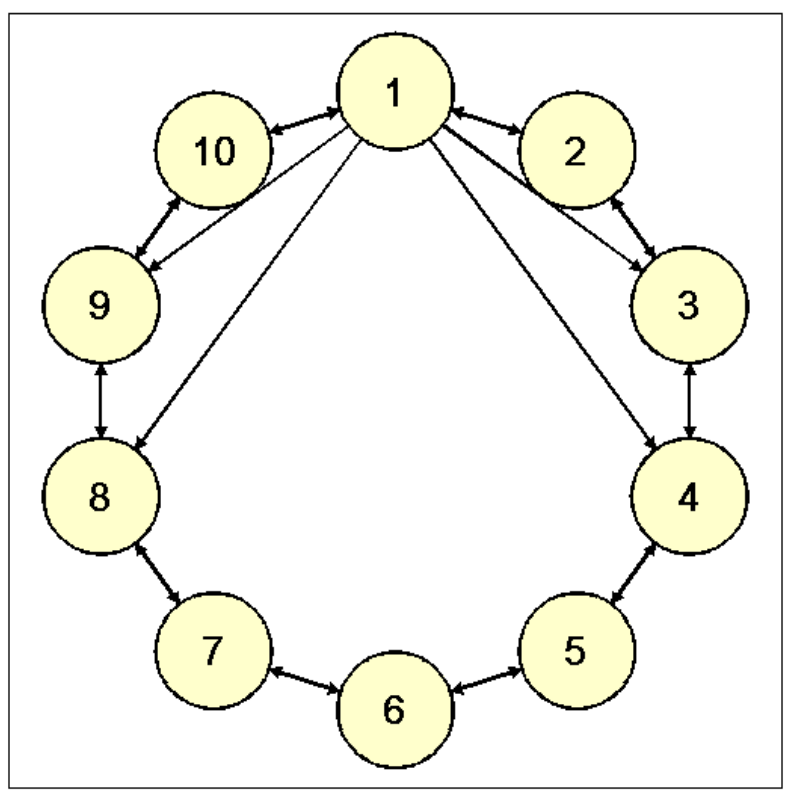

Figure 4.2. Nonlocal coupling illustrated for oscillator indexed $j=1$ with a coupling radius of $n$ $=3$. 
Figure 4.1 shows that increasing $\kappa$ decreases the coupling range and coupling strength of the oscillator. For high values of $\kappa$, each oscillator strongly interacts with its nearest neighbor. For $\kappa=$

2 , the effective coupling strength is approximately 8 times weaker than $\kappa=0.2$. Nonlocal coupling is an intermediate coupling between local coupling and gobal coupling. Increasing the value of $\kappa$ or decreasing the coupling radius shifts the behavior of the system toward a locally coupled system, while decreasing the value of $\kappa$ or as the coupling radius approaches $\frac{N}{2}$, where $\mathrm{N}$ is the population size, the system shifts toward becoming globally coupled. Random and special initial conditions were used in this investigation. With special initial conditions, a fraction of oscillators in the group are synchronized prior to introducing the nonlocal coupling scheme. Figure 4.2 shows a simplified representation of a nonlocally coupled network.

\subsection{Experiments}

Experiments were carried out using the photosensitive Belousov-Zhabotinky (BZ) system described in Chapter 2. The appropriate light perturbation $\phi_{j}$ from the SLM was calculated according to the coupling relation

$$
\phi_{j}=\phi_{0}+\sum_{\rho=j-n}^{j+n} K\left(I_{\rho}(t-\tau)-I_{j}(t)\right),
$$

where $\phi_{0}$ is the background light intensity [11], $\tau$ is a time delay [5] in the feedback from neighboring oscillator $\rho$ to oscillator $j, n$ is the coupling radius, and $j=1,2, \ldots, N$. The experiments were carried out with 40 oscillators in a ring configuration, coupled according to the coupling function $K$ defined in equation (4.1). 


\subsubsection{Chimera State from Quasi-Random Initial Phase Distribution}

Figure 4.3 shows an example of typical experimental behavior with 40 chemical oscillators. A snapshot of the phase of each oscillator at $t=1220 \mathrm{~s}$ is shown in Figure 4.3a. We see a group of synchronized oscillators with oscillator index $j=9-17$ and unsynchronized oscillators with $j=1-8$ and $j=30-40$. Also shown are oscillators that form a diagonal feature with $j=18-29$, which represents a wave of synchronized behavior. Video images show that the wave emanates from a group of unsynchronized oscillators, as discussed below [13].

Figure 4.3b shows the phase calculated from the measured gray level as a function of time for each of the oscillators. The spontaneous appearance of the group of synchronized oscillators can be seen at $t \approx 300 \mathrm{~s}$. The diagonal wave feature occurs at $t \approx 600-1080 \mathrm{~s}$, and, at $t \approx 1200-1400 \mathrm{~s}$, it transforms into a group of synchronized oscillators that are out of phase with the original synchronized group. Figure 4.3c shows the period of each oscillator for two different times, demonstrating that the simultaneous firing of the synchronized oscillators gives rise to a shorter oscillatory period than that of the unsynchronized oscillators.

The evolution of the chimera state in terms of the local order parameter $R[8]$, defined by

$$
R(j, t)=\frac{1}{2 m}\left|\sum_{\rho=j-m}^{j+m} \exp (i \theta(\rho, t))\right|,
$$

is shown in Figure 4.3d, where $j=1,2, \ldots, N$, and $m$ is the sampling radius. The value of $R$ is color coded such that red corresponds to large $R$ and high order and blue to small $R$ and low order. The high order of the original group of synchronized oscillators can be seen as well as the region of synchronization appearing at $t \approx 1080 \mathrm{~s}$. The remaining unsynchronized oscillators make up the surrounding regions of low order. Size oscillations of the synchronized group occur as the higher-frequency oscillators "lap" the unsynchronized oscillators, and neighboring oscillators transiently join the synchronized group when their phases align. 
Many nonlocal coupling experiments were carried out, some with quasi-random distributions of the initial phases and others with special initial conditions. In the first case, a group of synchronized oscillators typically appeared spontaneously, similar to the behavior shown in Figure 4.3b.

\subsubsection{Chimera State from Special Initial Conditions}

In experiments with special initial conditions, a group of synchronized oscillators was produced by using perturbations in illumination intensity, while the remaining oscillators had a quasi-random distribution of phases. In these experiments, the region of synchronization typically evolved in time, either disappearing with another synchronized region appearing or shifting to another region among the unsynchronized oscillators. Figure 4.4 shows an example of an experiment initiated from special conditions, where a synchronized group of oscillators with oscillator index $j=9-17$ coexists with unsynchronized oscillators with $\mathrm{j}=1-8$ and $j=18-30$. The time evolution of the phases in Figure 4.4b and the local order parameter in Figure 4.4d show size oscillations of the synchronized group of oscillators. Figure 4.4c shows distributions of the period at two different times.

\subsubsection{Synchronized States}

The photosensitive chemical oscillators have an inherent distribution in the oscillatory period that arises from the size distribution of the catalytic particles. The oscillator populations with this distribution allowed full and cluster synchronization of the oscillators in addition to the chimera state for the same conditions. Attaining the synchronized state ensured that a partially entrained state with synchronized and unsynchronized oscillators arising from an overly broad frequency distribution did not occur $[6,14]$. The fully synchronized stated and the synchronized clusters are 

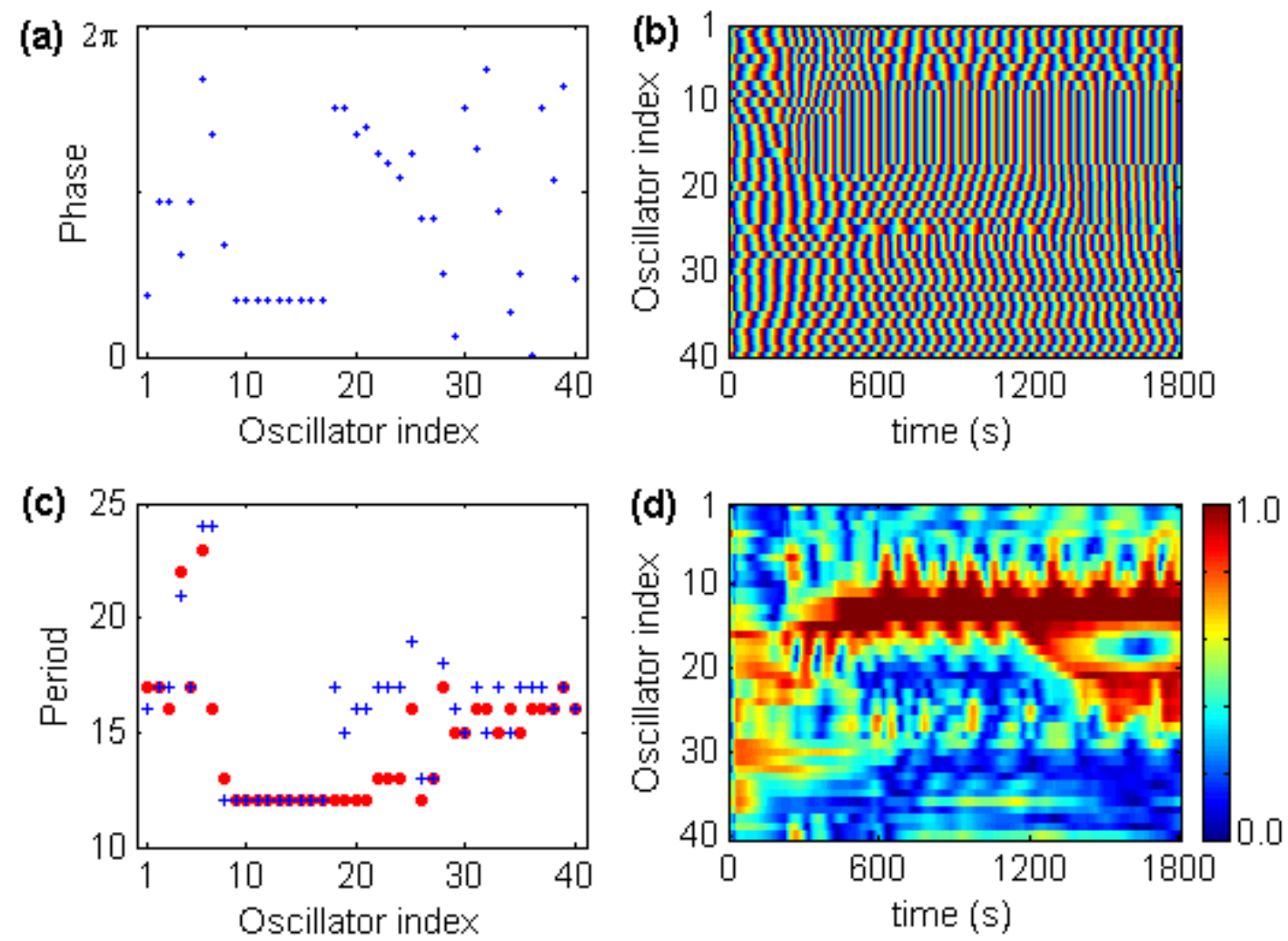

Figure 4.3. Experimental behavior of 40 oscillators coupled according to Eq. (4.2) in a ring configuration, with $n=10, \kappa=0.5, K^{\prime}=1, \tau=30 \mathrm{~s}$. The experiment was started with a quasi-random initial phase distribution of the oscillators. (a) Snapshot showing the phase of each oscillator at $t=1220 \mathrm{~s}$. (b) Phase of each oscillator as a function of time. (c) Period of each oscillator at $t=900($ blue +$), 1500($ red $\bullet)$ s. $(d)$ Local order parameter $R$ according to Eq. (4.3) as a function of time, with $m=3$. 
(a)

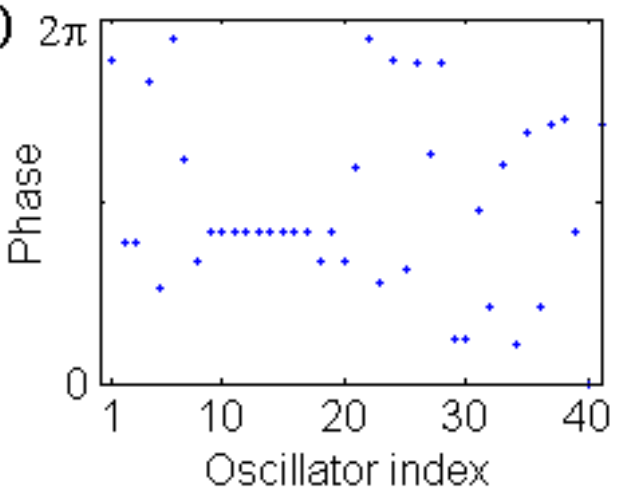

(c)

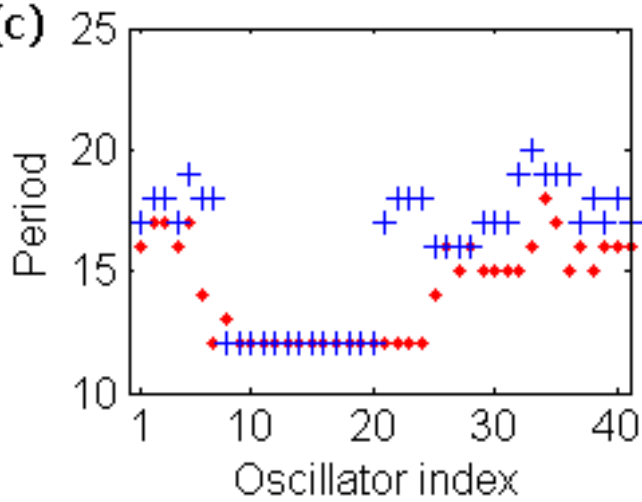

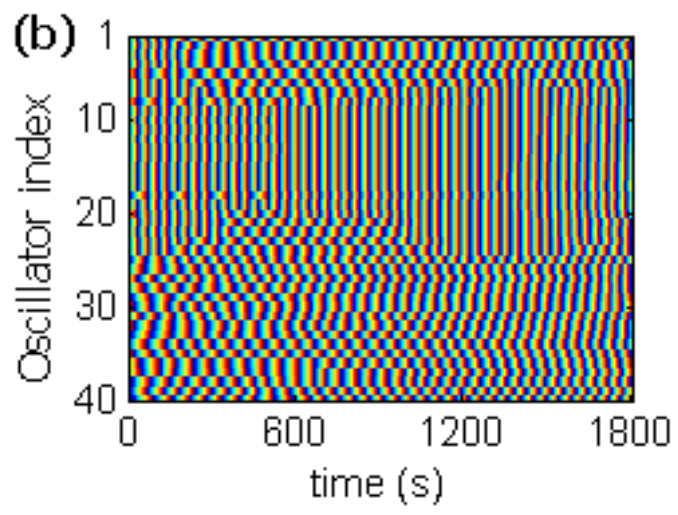

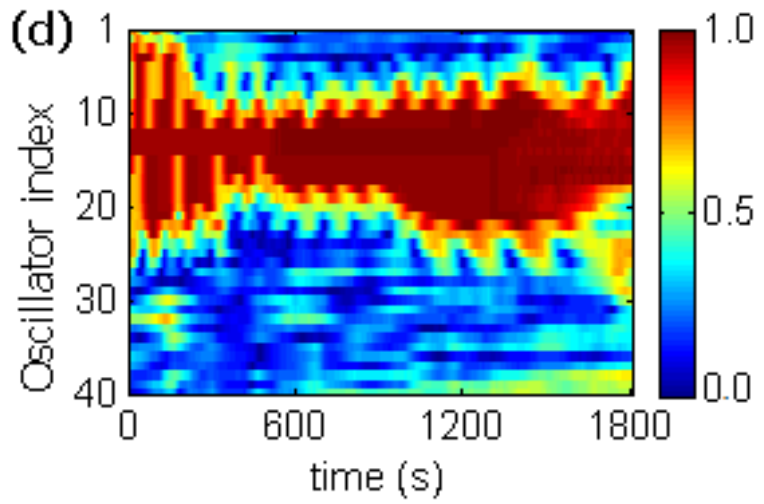

Figure 4.4. Experimental behavior of 40 oscillators coupled according to Eq. (4.2) in a ring configuration, with $n=10, \kappa=0.5, K^{\prime}=1, \tau=30 \mathrm{~s}$. The experiment was started with a special initial phase distribution of the oscillators. (a) Snapshot showing the phase of each oscillator at $t=1220 \mathrm{~s}$. (b) Phase of each oscillator as a function of time. (c) Period of each oscillator at $t=900($ blue +$), 1500($ red $\bullet$ ) s. (d) Local order parameter $R$ according to Eq. (4.3) as a function of time, with $m=3$. 
shown in Figure 4.5.

\subsubsection{Phase-Cluster Chimera States}

In addition to chimera states with phasewave behavior, we also find phase-cluster chimera states [5], where two or more out-of-phase groups of synchronized oscillators coexist with unsynchronized oscillators. Figure 4.6 shows a snapshot of the phase of each oscillator in an experimental phase-cluster chimera at $t=1206 \mathrm{~s}$. We see two clusters of synchronized oscillators having the same phase with $j=5-10$ and $j=22-29$ and a third out-of-phase cluster with $j=33-37$. The local order parameter $R$ as a function of time in Figure 4.6 shows that these clusters persist, and, interestingly, that the first cluster displays prominent antiphase size oscillations. The cluster grows in one direction by recruiting neighboring oscillators and then grows on the opposite side by recruiting these neighboring oscillators.

\subsubsection{Phase-Wave Chimera States}

In Figure 4.3, we see three types of behavior coexisting, in-phase synchronized, phase-wave behavior and unsynchronized groups of oscillators. Figure 4.7 shows experimental behavior of a frequency synchronized group of oscillators with a constant phase difference (phase wave) coexisting with an unsynchronized group of oscillators. The phase snapshot at $\mathrm{t}=1200 \mathrm{~s}$ shows oscillators indexed $j=1-7$ and $j=32-40$ in a wave-like structure and oscillators indexed $j=8-31$ are unsynchronized. The wave behavior seems to connect two groups of unsynchronized oscillators indexed $j=8-21$ and $j=22-31$. Analysis of the variations of the period as a function of time confirms that the behavior persists for at least 15 periods, as shown in Figure 4.7b. The plot of period as function of time for oscillator indexed $j=8$, belonging to the phase-wave group of 

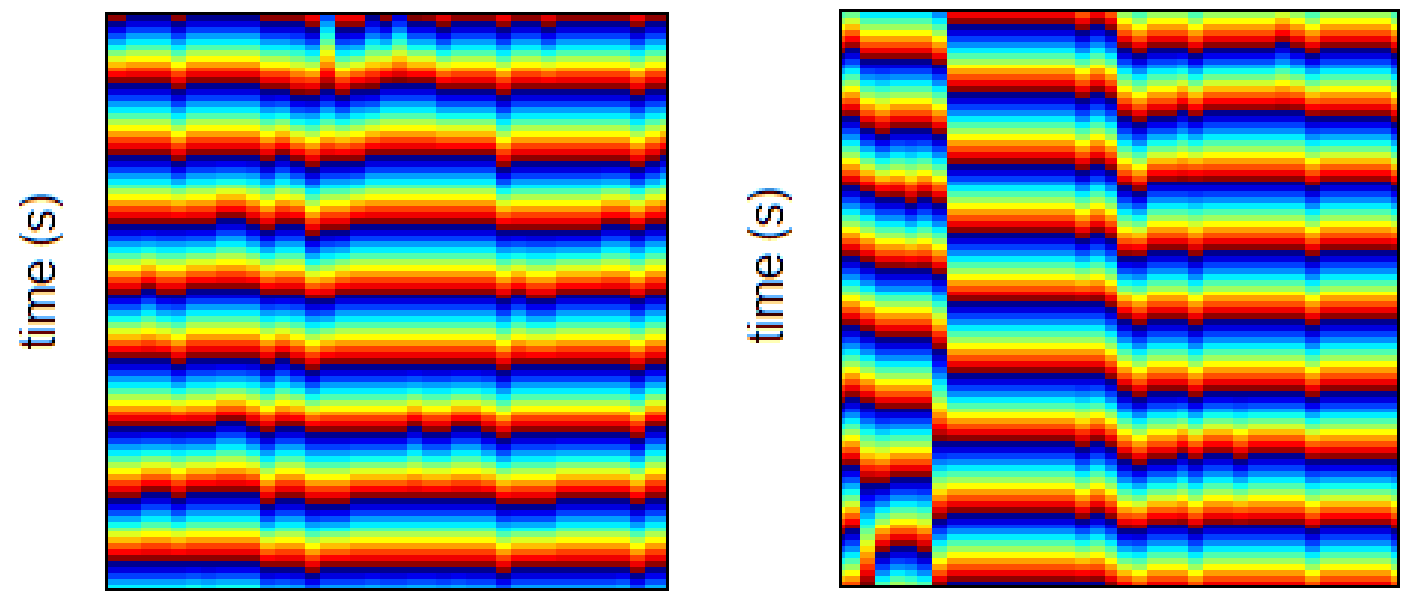

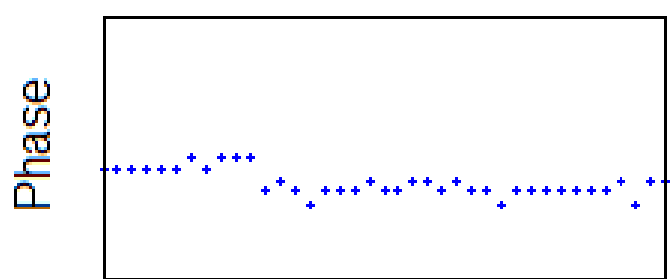

Oscillator index

(a)

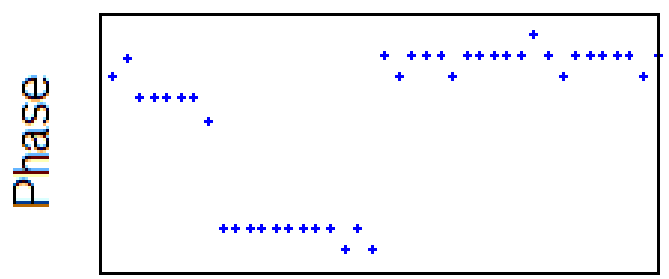

Oscillator index

(b)

Figure 4.5. Synchronized experimental behavior of 40 oscillators in a ring configuration, with $n=10, \kappa=0.5, K^{\prime}=1, \tau=30 \mathrm{~s}$. The experiment was started with a quasi-random initial phase distribution of the oscillators. (a) Phase snapshot of the fully synchronized state at $t=1220 \mathrm{~s}$ (bottom panel) and time evolution of the phases of the oscillators (top panel). (b) Snapshot of synchronized clusters, with the phase of each oscillator at $t=1220 \mathrm{~s}$ (bottom panel) and phases of the oscillators as a function of time (top panel). 
(a)

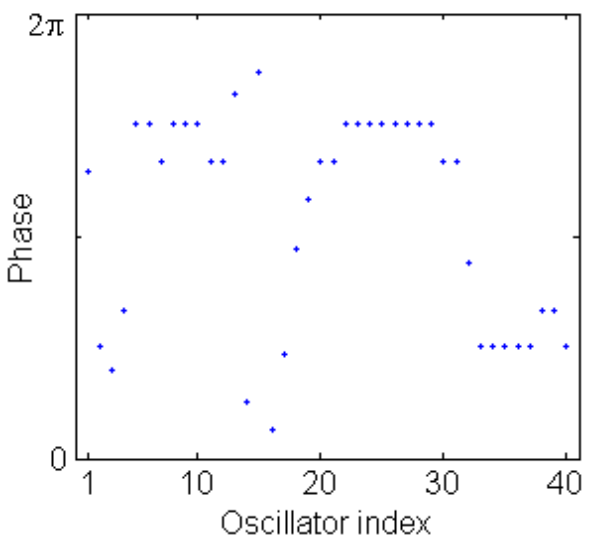

(b)

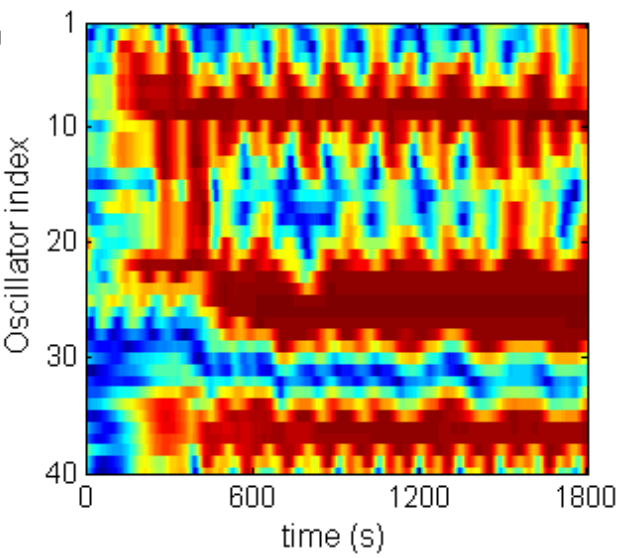

Figure 4.6. (a) Measurements showing experimental phase-cluster chimera in 40 coupled BZ oscillators, with $n=10, \kappa=0.8, K^{\prime}=1.0$, and $\tau=30.0$ s. The experiment was started with a quasi-random initial phase distribution; the snapshot shows the phase of each oscillator at $t=1206$ s. (b) Local order parameter $R$ of oscillators in (a) as a function of time, with $m=3$.

oscillators, shows an approximately constant period with small fluctuations due to experimental noise. The period evolution of oscillator indexed $j=18$, which belongs to the unsynchronized group, shows irregular variations with time. The period of the phase-wave synchronized oscillators is longer that the period of the in-phase synchronized oscillators but shorter than the average natural period of the oscillators.

\subsection{Simulations}

Simulations of the nonlocally coupled chemical oscillators were carried out using the twovariable ZBKE model for the BZ reaction [15], modified to describe the photosensitivity of the $\mathrm{Ru}(\mathrm{bpy})^{3+}$ catalyzed discrete oscillator system as described in Chapter 2:

$$
\phi_{j}=\phi_{0}+\sum_{\rho=j-n}^{j+n} K\left(Z_{\rho}(t-\tau)-Z_{j}(t)\right)
$$



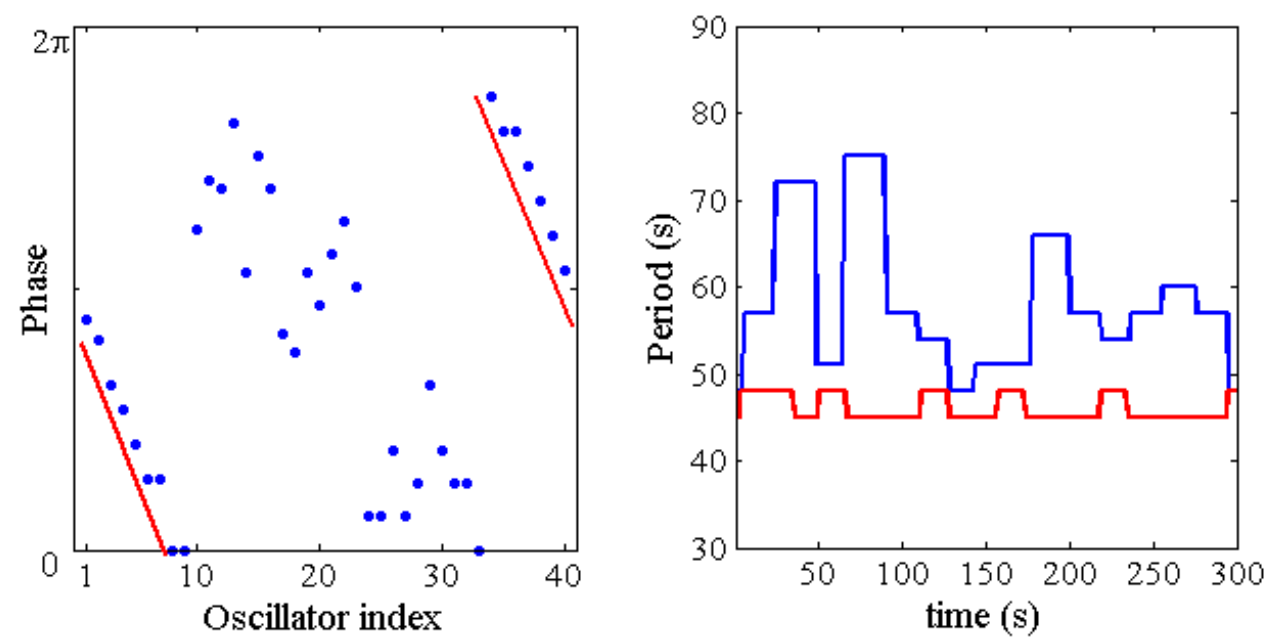

Figure 4.7. (a) A snapshot of experimental phase-wave chimera behavior at $t=600 \mathrm{~s}$. (b) Period as a function of time for the oscillators indexed $j=18$ (blue) and $j=38$ (red).

where $Z_{j}$ is the concentration of the oxidized form of the catalyst associated with the $j^{\text {th }}$ oscillator and the other parameters are the same as in Eq. (4.2).

\subsubsection{Chimera State}

Figure 4.8 shows behavior from a simulation of the nonlocally coupled BZ oscillator system, where a group of oscillators spontaneously synchronized at $t \approx 2.0 \times 10^{3}$ to form a chimera state of synchronized and unsynchronized oscillators. Figure 4.8a shows a snapshot of the phase of each oscillator at $t=1.90 \times 10^{4}$. This type of chimera behavior, with one relatively stable region of synchronized oscillators surrounded by unsynchronized oscillators, was seen frequently in our experiments and simulations. The phase of each oscillator as a function of time in Figure $4.8 \mathrm{~b}$ illustrates the stability of the synchronized group, but also illustrates the complex behavior of the unsynchronized oscillators, which tend to form transient diagonal wave features as well as transient in-phase or out-of-phase synchronization of a few oscillators. Figure 4.8c shows that the period 
of the synchronized oscillators is significantly shorter than that of the unsynchronized oscillators, much like the experimental systems shown in Figures 4.3c and 4.4c. Figure 4.8d illustrates the evolution of the chimera state according to the local order parameter $R$ in Eq. (4.3). We again see features that correspond to size variations of the synchronized group, although in this example the changes are irregular. We also note that within the unsynchronized oscillator region, small patches of fleeting behavior with slightly higher order can be discerned, corresponding to the transient synchronization shown in Figure 4.8b.

Several different types of chimera behavior were found in our experiments and simulations, including phase-cluster states and phase-wave chimera states.

\subsubsection{Phase-Cluster Chimera States}

Phase-cluster chimera state observed in the experiments described in Figure 4.4 were also seen in simulations. The phase-clusters spontaneously formed from initial random phase distributions or formed from splitting of the synchronized group. The splitting can be from a spontaneously formed synchronized group as in Figure $4.3 \mathrm{c}$ at $t \approx 1200-1400$ or from the initially synchronized group in experiments initiated from special conditions. Figure 4.9 shows a two-phase cluster state. Two out-of-phase clusters are clearly seen in Figure 4.9a and the persistence of the behavior is shown in Figure 4.9b. The behavior lasted for the entire duration of the simulation, although there were occassional cluster size fluctuactions. In Figure $4.9 \mathrm{~b}$, the cluster at $j=11-16$ increases during the time interval $t=2500$ - 2570. These size fluctuations are due to occassional 'lapping' discussed earlier. Once formed, the synchronized group did not significantly shift from the initial cluster. The sizes of the clusters varied significantly with the initial phase distribution. 

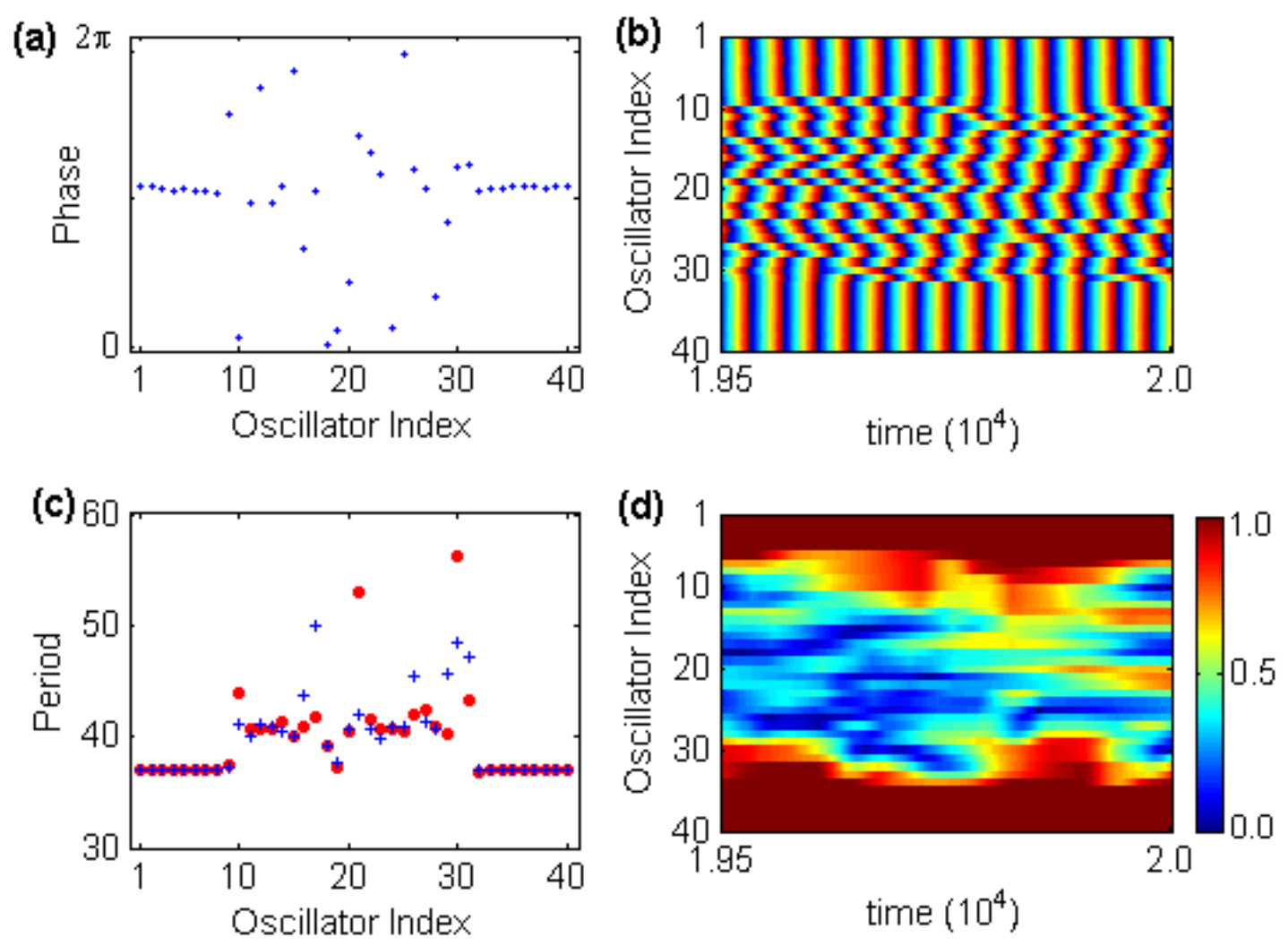

Figure 4.8. Model simulations of 40 coupled heterogeneous BZ oscillators in a ring configuration, with $n=10, \kappa=0.4, K^{\prime}=6.3 \times 10^{-5}, \tau=35.0$, and $\phi_{0}=1.6 \times 10^{-4}$. The simulation was started with a random initial phase distribution. (a) Snapshot showing the phase of each oscillator at $t=1.90 \times 10^{4}$. (b) Phase of each oscillator as a function of time. (c) Period of each oscillator at $t=1.85 \times 10^{4}($ blue +$), 1.90 \times 10^{4}($ red $\bullet) .($ d) Local order parameter $R$ as a function of time, with $m=3$. 

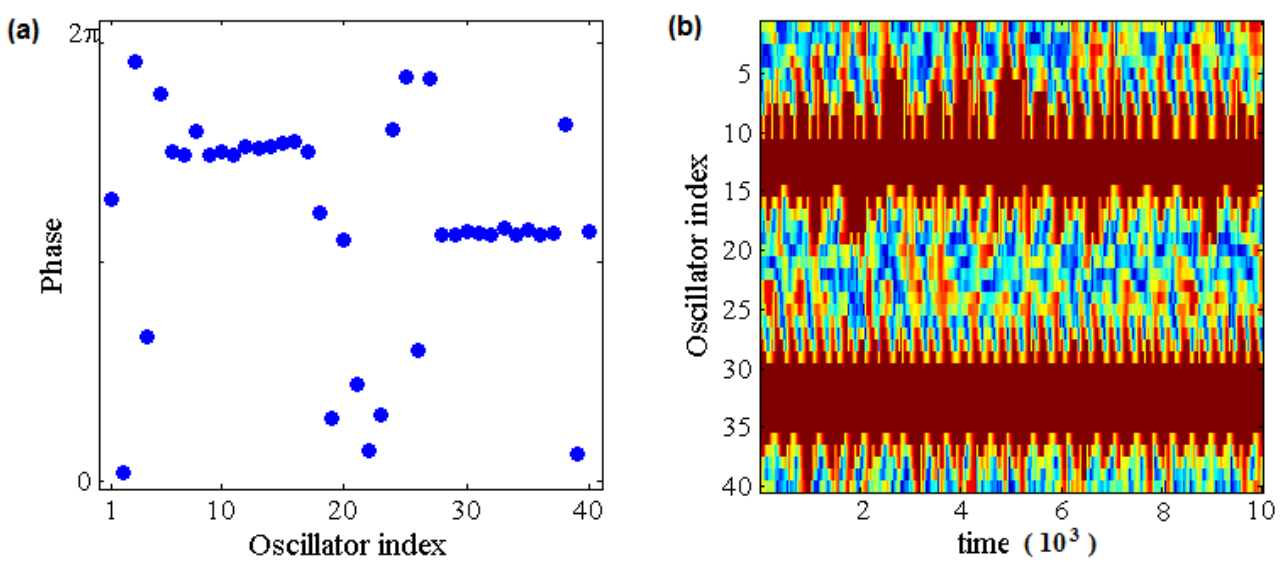

Figure 4.9. Model simulations of 40 coupled heterogeneous BZ oscillators in a ring configuration, with $n=10, \kappa=0.4, K^{\prime}=6.3 \times 10^{-5}, \tau=35.0$, and $\phi_{0}=1.6 \times 10^{-4}$. The simulation was started with a random initial phase distribution. (a) Snapshot showing the phase of each oscillator at $t=3.80 \times 10^{3}$. (b) Local order parameter $R$ as a function of time, with $m=3$.

\subsubsection{Phase-Wave Chimera States}

The phase wave behavior described in Figures 4.3 and 4.7 was also found in simulations, as shown in Figure 4.10a. The wave behavior is clearly seen in the snapshot of the oscillator phases at $t=1.93 \times 10^{4}$.

Video images [13] show that the waves are initiated from a small region of unsynchronized oscillators, with oscillator index $j=30-34$. On the right-hand side, the wave travels along the diagonal, $j=35-40$, where it continues through the periodic boundry to $j=1-9$. The wave on the left-hand side, $j=24-29$, travels to a group of unsynchronized oscillators, $j=10-23$, corresponding to the collision region of the two waves. The phase of each oscillator as a function of time shows the persistence of the wave features, Figure 4.10b. Complex behavior is again seen in the unsynchronized oscillators, with small groups becoming transiently synchronized in wave-like structures. 

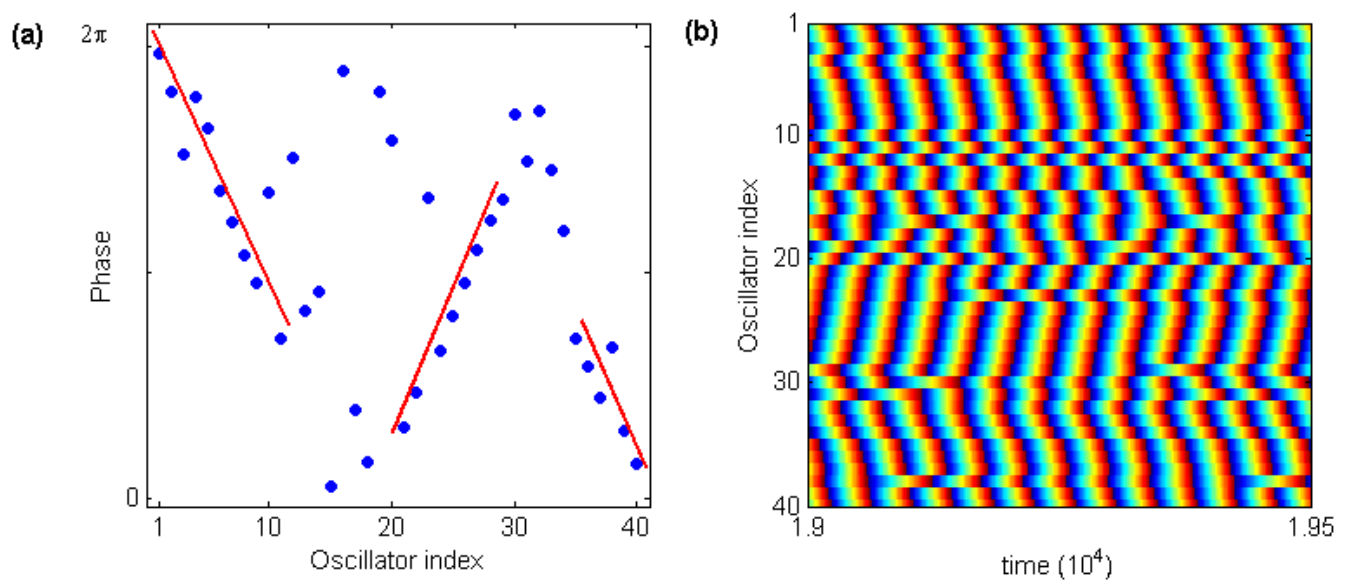

Figure 4.10. Simulations of phase-wave chimera, where the red lines have been added to guide the eye. Model simulations of 40 coupled heterogeneous BZ oscillators, with $n=10, \kappa=0.4$, $K^{\prime}=6.3 \times 10^{-5}, \tau=35.0$, and $\phi_{0}=1.6 \times 10^{-4}$. The simulation was started with a random initial phase distribution. (a) Snapshot shows phase of each oscillator at time $t=1.93 \times 10^{4}$. (b) Phase of each oscillator in (a) as a function of time. 


\subsection{Spiral Chimera States}

Simulations based on the photosensitive BZ oscillator model have also been carried out in a planar two-dimensional configuration. We use the same nonlocal coupling, with the separation of the oscillators determined by the Pythagorean distance according to the oscillator indices in the square lattice, which is typically made up of $50 \times 50$ oscillators. The spiral cores of asynchronous oscillators meander in an irregular manner, with the form of the meandering sensitive to the value of the delay $\tau$. Figures $4 \mathrm{a}$ and $4 \mathrm{~b}$ show snapshots of pairs of initially symmetrical counter-rotating spirals at $t=3500$ for two slightly different values of $\tau$. A 2D local order parameter can be calculated through generalization of Equation (4.3), and the value of $R$ at each point in Figures 4.11a and $4.11 \mathrm{~b}$ is shown in Figures 4.11c and 4.11d. The meander of the asynchronous core is tracked by following the minimum in $R$, and Figure 4.11c, corresponding to the nearly symmetrical spiral pair in Figure 4.11a, illustrates the case in which the asynchronous cores undergo approximately random-walk behavior. We have observed cases in which the mean-squared displacement is linear with time, although we also find deviations from this behavior. Figure 4.11d, corresponding to the asymmetric spiral pair in Figure 4.11b, shows larger irregular motions of the cores of asynchronous oscillators. The irregular motion in both cases is similar to that of reaction-diffusion spiral cores in the presence of spatiotemporal noise imposed on the medium excitability [16].

The irregular meandering behavior arises from the interaction of the core of asynchronous oscillators with the spiral wave tip. As the tip rotates, it experiences different asynchronous oscillators, which, depending on the oscillator phase, cause the tip to grow or contract. The interaction may occur with more than the outer "boundary" asynchronous oscillators when transient phase alignment occurs with the interior oscillators, leading to larger fluctuations of the spiral tip. Changes in the spiral tip also lead to distortions of the shape of the core of asynchronous oscillators. 


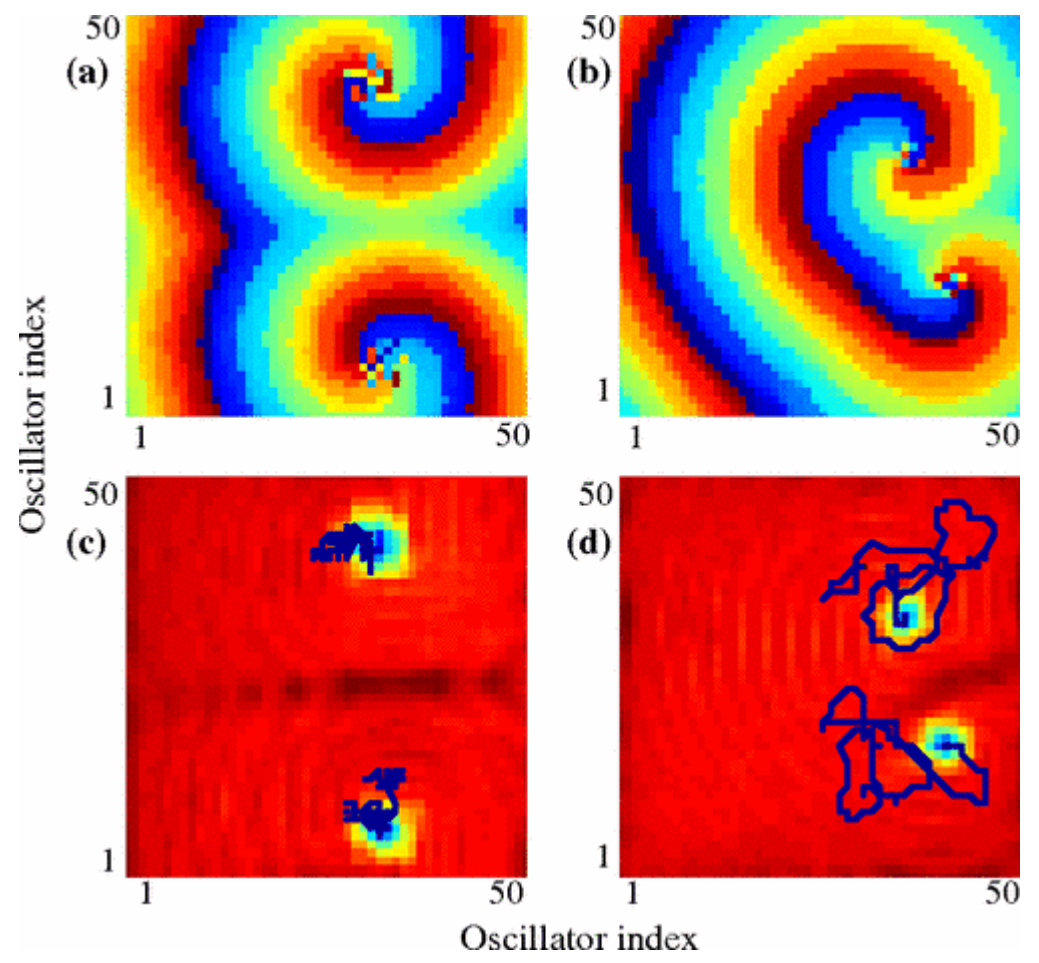

Figure 4.11. Simulations of spiral chimera states in populations of BZ oscillators. The system is composed of $50 \times 50$ oscillators in a square lattice configuration, with a coupling radius of $n=4$ and fixed boundary conditions. Top images show the phase of each oscillator in the lattice at $t=3500$ for values of delay $\tau=4.0$ (a) and 3.4 (b). Each simulation is initiated with a pair of symmetric counter rotating spirals, with $\tau=0$. The delay is switched on at $t=500$ and the simulation is continued to $t=3500$. Images (c) and (d) show the local order parameter $R$ at $t=3500$. The dark blue line shows the trajectory of the minimum in $R$ between $t=700$ and 3500. Parameters: $\kappa=0.3, K^{\prime}=1.4 \times 10^{-3}$, and $\phi_{0}=1.1 \times 10^{-4}$. 


\subsection{Summary}

Chimera, phase-wave, phase-cluster chimera states, and synchronized states were observed in both experiments and simulations. In addition to these states, coexisting groups of in-phase synchronized, phase-wave synchronized, and asychronous oscillators were observed. These states are further analyzed in Chapter 5. The spontaneous formation of chimera states from quasirandom initial phase distributions in experiments and random phase distributions in simulations suggests that the initial conditions do not play a crictical role in the formation of chimera states in our system. In the previous work, initial conditions were key to the observation of chimera states in the two-group model. Most studies, including the original chimera work by Kuramoto et. al [1] used populations of homogeneous oscillations. The differences in some of the observed behavior could be related to the frequency distribution in our system and the relaxation nature of the oscillations. Studies, which include systems of homogeneous chemical oscillators are discussed in the next chapter.

Experiments and modeling studies of chimera behavior in 1D ring configurations provide insights into spiral chimera behavior in 2D. The origin of wave behavior in both $1 \mathrm{D}$ and $2 \mathrm{D}$ is a group of asynchronous oscillators. The 1D out-of-phase wave initiation shown in Fig. 4.10a is analogous to the 2D spiral wave behavior shown in [13]. The size variations of the groups of synchronized oscillators seen in both 1D experiments and simulations are related to the meandering behavior of the core of asynchronous oscillators in 2D reported in [13]. The phase-wave behavior in Fig. 4.7 is possibly transient behavior leading to splay states. 


\section{References}

[1] Y. Kuramoto and D. Battogtokh, "Coexistence of coherence and incoherence in nonlocally coupled phase oscillators," Nonlinear Phenom. in Complex Syst. 5, 380-385 (2002).

Online Version 66, 84

[2] S. Shima and Y. Kuramoto, "Rotating spiral waves with phase-randomized core in nonlocally coupled oscillators," Phys. Rev. E 69, 036213 (2004).

Online Version 66

[3] D. M. Abrams and S. Strogatz, "Chimera states for coupled oscillators," Phys. Rev. Lett. 93, $174102(2004)$.

Online Version 66

[4] D. M. Abrams and S. H. Strogatz, "Chimera states in a ring of nonlocally coupled oscillators," Int. J. Bifurcation Chaos 16, 21-37 (2005).

Online Version 66

[5] G. C. Sethia, A. Sen, and F. M. Atay, "Clustered chimera states in delay-coupled oscillator systems." Phys. Rev. Lett. 100, 144102 (2008).

Online Version 66, 69, 74

[6] D. M. Abrams, R. Mirollo, S. H. Strogatz, and D. A. Wiley, "Solvable model for chimera states of coupled oscillators," Phys. Rev. Lett. 101, 084103 (2008).

Online Version 71

[7] A. E. Motter, "Spontaneous synchrony breaking," Nature Physics 6, 164-165 (2010). Online Version 
[8] I. Omelchenko, Y. Maistrenko, P. Hövel, and E. Schöll, "Loss of coherence in dynamical networks: Spatial chaos and chimera states." Phys. Rev. Lett. 106, 234102 (2011). Online Version 66, 70

[9] M. J. Panaggio and D. M. Abrams, "Chimera states on a flat torus," Phys. Rev. Lett. 110, 094102 (2013).

Online Version 66

[10] A. M. Hagerstrom, T. E. Murphy, R. Roy, P. Hövel, I. Omelchenko, and E. Schöll, "Experimental observation of chimeras in coupled-map lattices," Nature Physics 8, 658-661 (2012). Online Version 67

[11] M. R. Tinsley, S. Nkomo, and K. Showalter, "Chimera and phase-cluster states in populations of coupled chemical oscillators," Nature Physics 8, 662-665 (2012).

Online Version 67, 69

[12] E. A. Martens, S. Thutupalli, A. Fourrière, and O. Hallatschek, "Chimera states in mechanical oscillator networks." PNAS. 110, 10563-7 (2013).

Online Version 67

[13] S. Nkomo, M. R. Tinsley, and K. Showalter, "Chimera states in populations of nonlocally coupled chemical oscillators," Phys. Rev. Lett. 110, 244102 (2013).

Online Version 70, 80, 84

[14] A. Winfree, "Biological rhythms and the behavior of populations of coupled oscillators." J. Theor. Biol. 16, 15-42 (1967).

Online Version 71 
[15] A. M. Zhabotinsky, F. Bucholtz, A. B. Kiyatkin, and I. R. Epstein, "Oscillations and waves in metal-ion-catalyzed bromate oscillating reactions in highly oxidized states," J. Phys. Chem. 97, 7578-7584 (1993).

Online Version 76

[16] I. Sendiña Nadal, S. Alonso, V. Pérez-Muñuzuri, M. Gómez-Gesteira, V. Pérez-Villar, L. Ramírez-Piscina, J. Casademunt, J. Sancho, and F. Sagués, "Brownian motion of spiral waves driven by spatiotemporal structured noise," Phys. Rev. Lett. 84, 2734-2737 (2000). Online Version 82 


\section{Chapter 5}

\section{Analysis of Dynamical Regimes in Coupled Chemical Oscillators}

\subsection{Introduction}

Chimera states in nonlocally coupled oscillators have been found in populations of oscillators with different types of dynamical behavior, which include phase oscillators $[1,2,4 ?]$, chemical oscillators [5-7], and model neuron oscillators [8, 9]. Variations from the typical classical chimera have been observed through modifications to the coupling structure, such as including delay and changing coupling strength. Phase-cluster chimera states, with multiple coherent regions, have been observed in systems with delay $[5,6,10-13]$, strong coupling $[8,14]$ and also with a piecewise linear nonlocal coupling function $[15,16]$. In these states, adjacent coherent regions have opposite phases. Phase-wave chimera states have been observed in heterogeneous populations of chemical oscillators [5]. These are made of oscillators forming a coherent wave structure coexisting with an incoherent group. Nonstationary chimeras have recently been reported in numerical investigations of the nonlocal, strongly coupled complex Ginzburg-Landau equation (NLCGE) [14]. In addition 
to amplitude variations associated with the strong coupling, drifting of the coherent-incoherent patterns were reported with a two-cluster chimera. As a result of the coherent unidirectional drift around a ring configuration, each oscillator exhibits coherent or incoherent behavior depending on the sampling time.

A ring network of oscillators with a coupling relation described by the equation (4.1) was used in the work described here. In Chapter 4, experiments and simulations were carried out with 40 oscillators. Here, we discuss the different dynamical regimes observed as $\kappa$ and $\mathrm{N}$ are varied independently in populations of chemical oscillators. The numerical investigation includes populations of homogeneous oscillators, each of which has the same frequency. We use different approaches to characterize the dynamical regimes, which include plots of the mean period, next return maps, local order parameter plots, and Fourier transformations. Detailed descriptions of synchronized, phase dispersed (PD), chimera, periodic switching, and drifting chimera states are given in Section 5.4 .

\section{$5.2 \quad$ Varying $\kappa$}

\subsubsection{Summary of Experiment Results}

In experiments, $\kappa$ was varied from $\kappa=0.2-1.0$ (see Eq. (4.1)). Figure 5.1 shows a summary of the dynamical states observed in the experiments. Synchronized states were the dominant behavior observed for $\kappa=0.2-0.3$, chimera states for $\kappa=0.4-0.7$, and unsynchronized states for $\kappa=$ 0.8 - 1.0. The fully synchronized states have all the oscillators phase-locked into one group or in phase-clusters (n-cluster state). Figure 5.1(a) shows a snapshot of the fully synchronized 1-cluster state. The oscillators have approximately the same period, as inferred from the time evolution of the phase in Figure 5.1(a)(ii), which also shows the persistence of the behavior. Synchronized 
(a) (i)

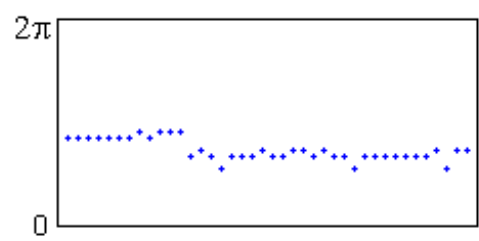

(b) (i)

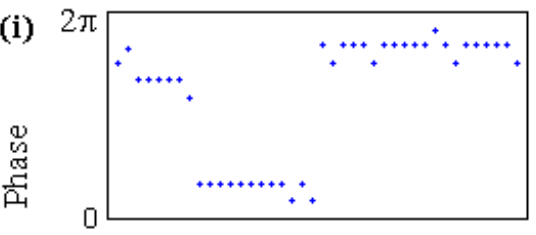

(c) (i)

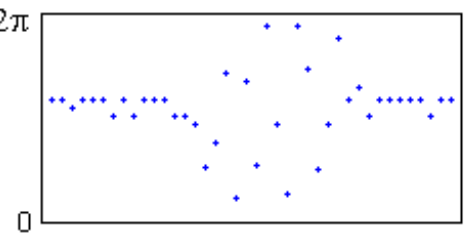

(d) (i)

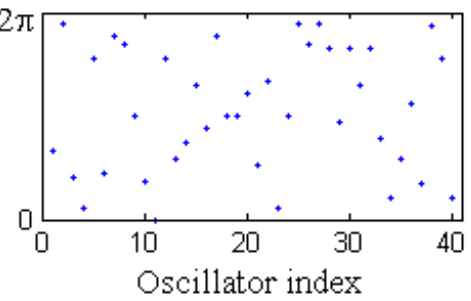

(ii)
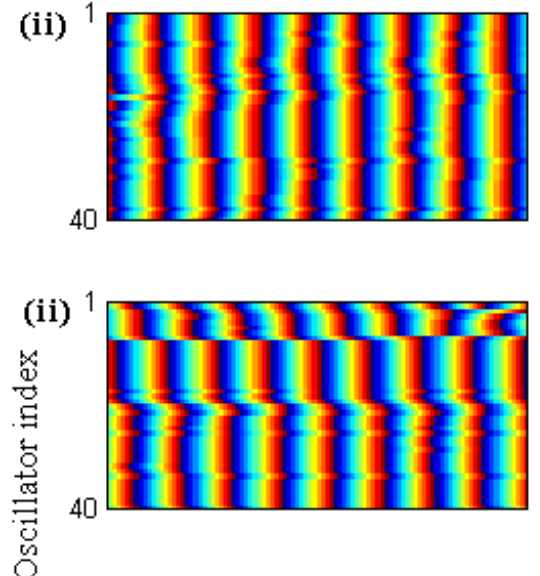

(ii)

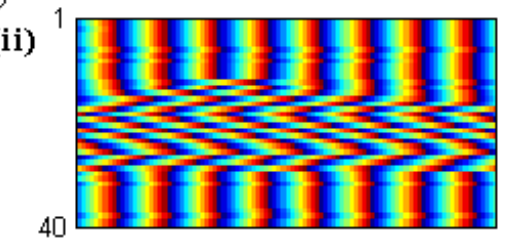

(ii)

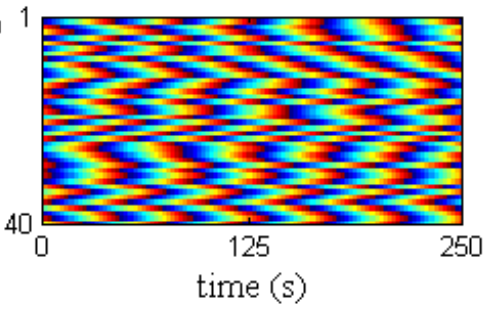

Figure 5.1. Summary of dynamical states observed in experiments. (a) Fully synchronized state, (b) phase-cluster state, (c) chimera state, and (d) the unsynchronized state.

phase clusters were observed for $\kappa=0.3$ - 0.4. In phase-cluster states, oscillators are permanently locked in their synchronized groups and all oscillators have close to the same period. Figure 5.1(b) shows a phase-cluster state with 40 oscillators. Oscillators indexed $j=1-8$ and $j=21-40$ form one cluster, while oscillators $j=9-20$ make up the other cluster, as shown in Figure 5.1(b)(i). The corresponding phase as function of time is shown in Figure 5.1(b)(ii), which shows an interval of eight periods.

Chimera behavior is characterized by synchronous group(s) of oscillators coexisting with un- 
synchronized oscillators. Typical experimental chimera behavior is shown in Figure 5.1(c). Figure 5.1(c)(i) shows a snaphot of a chimera state with synchronized oscillators, $j=1-12$ and $j=32-40$, and random phases for oscillators $j=13-31$. The corresponding Figure 5.1(c)(ii) shows the persistence of the chimera behavior. For high $\kappa$, the oscillators exhibit a distribution of phases and unsychronized phase-drifting behavior with time, as shown in Figures 5.1(d)(i) and (ii), respectively. High $\kappa$ corresponds to weak interactions between the oscillators; hence, the behavior is largely asynchronous. The phase drifting is a consequence of heterogeneity of the oscillators in the experiments.

\subsection{Simulation Results}

\subsubsection{Varying $\kappa$}

The behavior varied from complete synchronization to an asychronous state with increasing $\kappa$. In Figure 5.2(a), the homogeneous system shows a high likelihood of finding fully synchronized states for $\kappa=0.2-0.3$, and the phase dispersed state (PD) is the dominant behavior for $\kappa \geq 0.4$. With high $\kappa$, the interactions are too weak to bring the oscillators together in phase. However, the oscillators exhibit phase locking behavior since they have an identical natural frequency. For $\kappa=0.4$, chimera-like states were found in only 2 out of 50 realizations. Random initial phase alignment of nearest neigbhors contributes to the initiation of the synchronized behavior of these chimera-like states. The observations show a dependence of the occurence of chimera-like states on the initial phase distribution, which was reported in earlier studies $[1,17 ?]$.

In heterogeneous systems, the chimera-like states exist for $\kappa=0.2-0.7$ and they occur more often compared to the homogeneous system, as shown in Figure 5.2(b). Occasional phase alignment of oscillators with different frequencies increases the likelihood of initiating synchronized behav- 

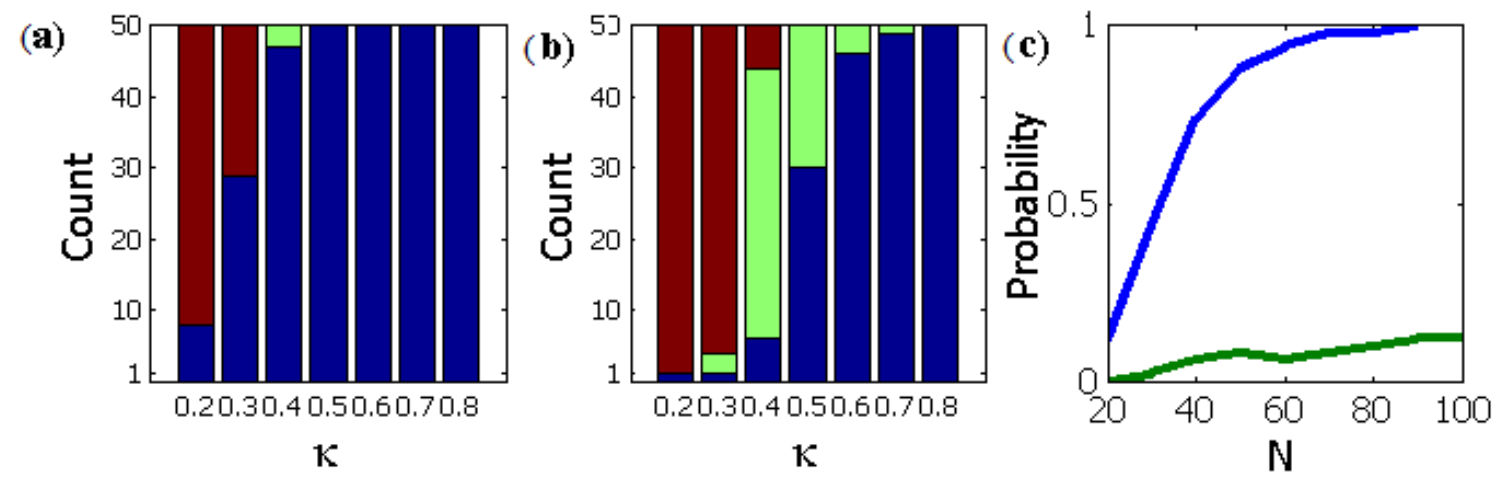

Figure 5.2. The likelihood of finding a chimera-like states as a function of coupling strength; (a) homogeneous oscillators and (b) heterogeneous oscillators; fully synchronized state (red), chimeralike states (green), asynchronous state (blue). Simulations were initiated from a random phase distribution of 40 oscillators. Counts are from 50 different initial random distributions per each value of K. (c) The probability of finding chimera-like states in systems of homogeneous (green) and heterogeneous (blue) oscillators. 
ior. As shown in Figures 5.2(a) and 5.2(b), the chimera state coexists with a fully synchronized state. Table 4.1 summarizes the dynamical behavior observed in simulations and experiment. The chimera-like states include the classical chimera, phase-cluster chimera, periodic switching or phase slipping behavior, and drifting phase-cluster chimera states.

\subsubsection{Varying $\mathrm{N}$}

The effect of group size on the likelihood of finding chimera-like states was investigated with both homogeneous and heterogeneous groups of oscillators. N was varied while keeping the coupling radius, $\rho=10$, and the coupling constant, $\kappa=0.4$, the same. The value of $\kappa$ chosen corresponds to the region with a high probability of finding chimera-like states in both systems of oscillators, as can be seen in Figures 5.2(a) and 5.2(b). The probability of finding a chimera-like state with varying $\mathrm{N}$ is shown in Figure 5.2(c). There was no significant change in the probability of finding chimera-like states in groups of homogeneous oscillators; for $20 \leq N \leq 100$ the probability was always less than 0.2 .

In heterogeneous systems, the probability of finding chimera-like states was observed to increase with system size for $20 \leq N \leq 80$. Only synchronized states were observed for $20 \leq N \leq 30$. For

this range of $\mathrm{N}$, the system's behavior is close to that of a globally coupled system $\left(\rho=\frac{N}{2}\right)$. The graph plateaus for $\mathrm{N} \geq 80$ oscillators, which means that the occurrence of the chimera-like states becomes less dependent on system size for large N. 
Table 5.1. Summary of the different observed behaviors

\begin{tabular}{|c|c|c|c|}
\hline$\kappa$ & Homogeneous system & Heterogeneous system & Experimental system \\
\hline 0.2 & 1-Cluster, n-Cluster & 1-Cluster & 1-Cluster \\
\hline 0.3 & $\begin{array}{l}\text { 1-Cluster } \\
\text { n-Cluster } \\
\text { Phase slip }\end{array}$ & $\begin{array}{l}\text { 1-Cluster } \\
\text { n-Cluster } \\
\text { Switchers }\end{array}$ & $\begin{array}{l}\text { 1-Cluster } \\
\text { 1-Cluster }\end{array}$ \\
\hline 0.4 & $\begin{array}{c}\text { 1-Cluster } \\
\text { n-Cluster } \\
\text { Phase slip } \\
\text { Chimera } \\
\text { Drifting chimera }\end{array}$ & $\begin{array}{c}\text { 1-Cluster } \\
\text { Chimera } \\
\text { Switchers } \\
\text { Phase wave chimera } \\
\text { 1-Cluster }\end{array}$ & $\begin{array}{l}\text { 1-Cluster } \\
\text { Chimera } \\
\text { Switchers }\end{array}$ \\
\hline 0.5 & $\begin{array}{c}\text { 1-Cluster } \\
\text { Phase dispersed }\end{array}$ & $\begin{array}{c}\text { Chimera } \\
\text { Phase dispersed }\end{array}$ & $\begin{array}{l}\text { Chimera, 1-Cluster } \\
\text { Phase dispersed }\end{array}$ \\
\hline 0.6 & $\begin{array}{c}\text { 1-Cluster } \\
\text { Phase dispersed }\end{array}$ & $\begin{array}{c}\text { Chimera } \\
\text { Phase dispersed }\end{array}$ & $\begin{array}{c}\text { Chimera } \\
\text { Phase dispersed }\end{array}$ \\
\hline 0.7 & Phase dispersed & Phase dispersed & Chimera, Phase dispersed \\
\hline $0.8-1.0$ & Phase dispersed & Phase dispersed & Phase dispersed \\
\hline
\end{tabular}




\subsection{Analysis of Experimental and Numerical Results}

\subsubsection{Phase Dispersed State}

We use "phase dispersed" state to describe a state where the oscillators have a distribution of phases and each oscillator exhibits periodic behavior (natural period). The description distinguishes the phase dispersed (PD) state from an asynchronous state, which we use to describe phase dispersed oscillators exhibiting aperiodic behavior. Phase dispersed states were observed for $\kappa \geq 0.8$ in simulations, using either homogeneous or heterogeneous oscillators, and in experiments. In experiments (Figure 5.3(a)) and simulations of heterogeneous oscillators (Figure 5.3(b)), the phase relations of the oscillators change with time, since the oscillators have different periods. The phase distributions shown in the snapshots in Figure 5.3(a)(i) and 5.3(b)(i) are, therefore, not stationary. Each oscillator in the group exhibits its natural periodic behavior. The patches of high local order parameter values (orange to red color) in Figures 5.3(a)(i) and (b)(i) are a result of occasional phase alignment of neighboring oscillators, as the faster oscillators 'lap' the slower oscillators. In homogeneous systems, the oscillators have randomly distributed phases, as shown in Figure 5.3(c)(i). A plot of the time dependent local order parameter shows constant values for each oscillator, which indicates that the oscillators are phase-locked.

\subsubsection{Synchronized States}

The fully synchronized states are associated with strong coupling and smaller group sizes. Synchronized states in populations of heterogeneous oscillators are discussed in Section 4.3.3. Figure 5.4(a) shows phase clusters in a system of 40 homogeneous BZ oscillators. The snapshot shows a 2-phase cluster state with oscillators $j=9-29$ forming one cluster and the remainder of the oscillators making up the second cluster. The blue line shows a snaphot of the local order 
(a) (i)

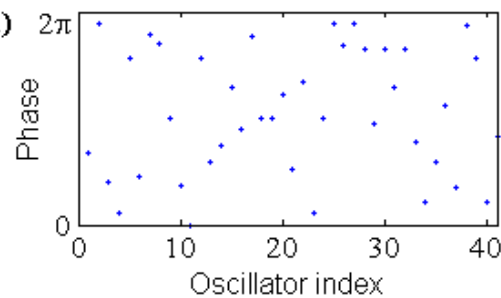

(b)

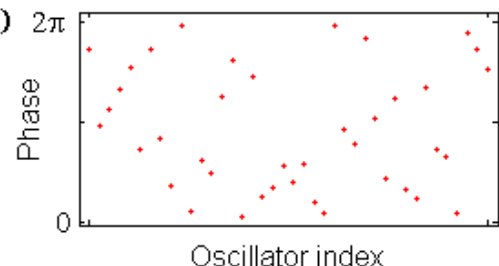

(c) (i)

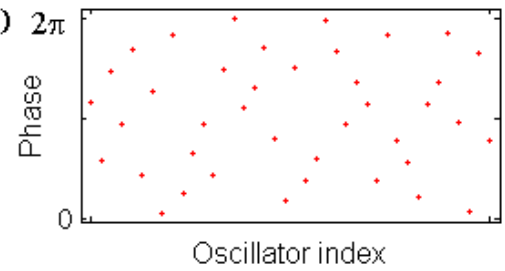

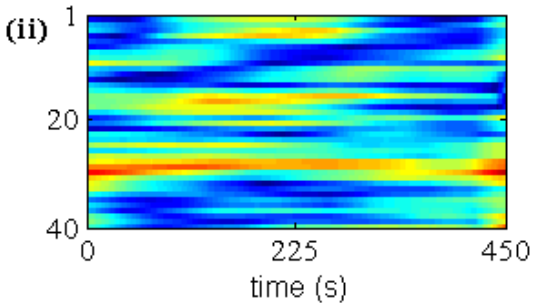

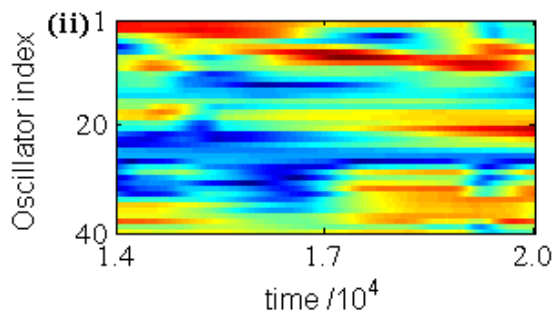

(ii)

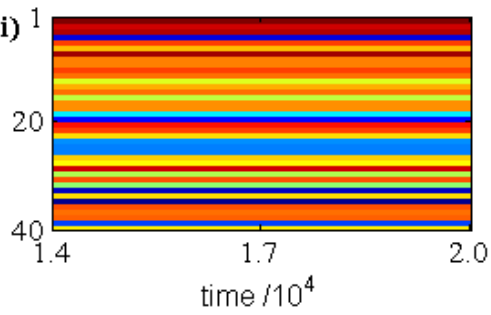

Figure 5.3. (a) Phase dispersed state in the experimental system. The experiment was started with a quasi-random phase distribution. (b), (c) Model simulations with 40 BZ oscillators. The simulations were started with a random phase distribution. (b) Phase dispersed state in heterogeneous oscillators. (c) Phase dispersed state in homogeneous oscillators 

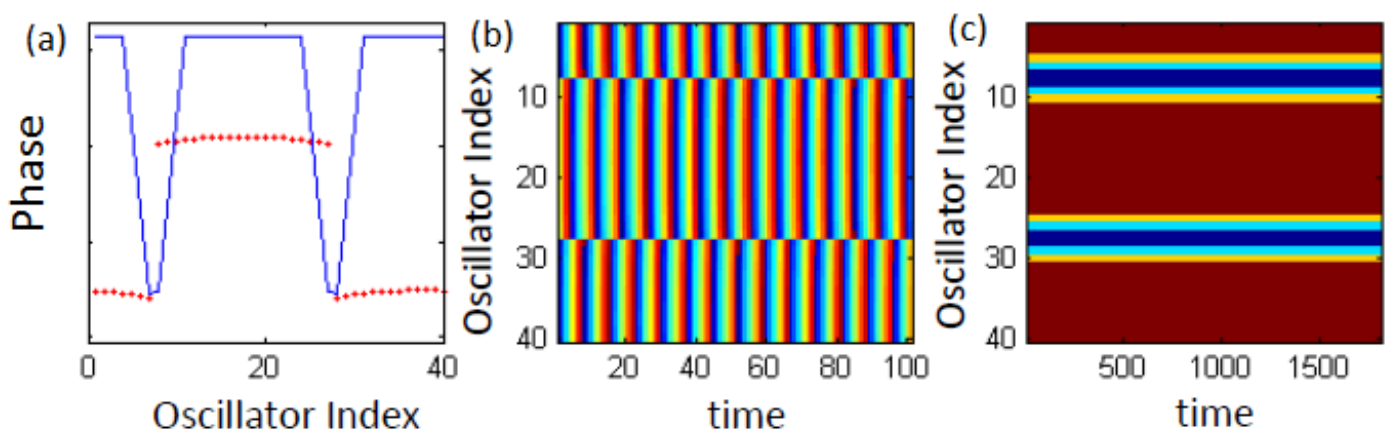

Figure 5.4. Phase-cluster state from model simulation of 40 homogeneous BZ oscillators. (a) A snapshot of the phase of each oscillator (red dots) and local order parameter (blue line) at time $t=1.8 \times 10^{4}$. (b) Phases of the oscillators with time. (c) Local order parameter values of each oscillator as a function of time. The simulation was started with a random initial phase distribution.

parameter, calculated using Eq. (4.3). The clusters are $\pi$ out of phase with each other, as shown in Figure 5.4(b). Oscillators $j=9-11$ and $j=29-31$ have lower local order parameter values because they are out of phase with their neighbors. The local order parameter used in this description (Eq. (4.3)) quantifies the level of in-phase synchrony. However, a constant R value provides information about the phase relations between neighboring oscillators (Figure 5.4 (c)). Phase-cluster states exist in the same parameter region as the fully sychronized state in both populations of homogeneous and heterogeneous oscillators

\subsubsection{Chimera States}

\subsubsection{Chimera States in Experiments}

Figure 5.5(a) shows a snapshot of a chimera state with synchronized oscillators, $j=12-19$, coexisting with an asynchronous group, $j=1-11$ and $20-40$. The experiment was started with 
a special initial phase distribution, with oscillators $j=1-20$ synchronized, as shown in Figure 5.5(b). The nonlocal coupling was introduced at $\mathrm{t}=300 \mathrm{~s}$. The synchronized group evolved to an optimum size and the behavior persisted for the duration of the experiment. A scatter plot of 25 periods of each oscillator in Figure 5.5(c) shows distributions of periods for asynchronous oscillators. The period of the synchronized oscillators was approximately the same for the duration of the experiment.

Figure $5.5(\mathrm{~d})$ shows power spectra of three of the oscillators in the system. Oscillator $j=15$ (blue) exhibits synchronous behavior and has narrow peaks compared to the broader peaks of the asynchronous oscillators $j=11$ and 23. Oscillator $j=23$ (green) has a smaller signal at the dominant frequency of oscillator $j=15$, which confirms some entrainement by the synchronized group. In contrast, oscillator $j=11$ does not show siginificant entrainment by the neighboring synchronized group. The spectra of the asynchronous oscillators exhibit a spread in frequency not observed in the spectrum of the oscillator $j=15$. The insert shows an expanded view of the power spectrum of oscillator $j=11$.

The distributions of periods and the Fourier transform analysis have confirmed the presence of coexisting subpopulations of synchronous and asynchronous behavior in the experiments.

\subsubsection{Chimera States in Homogeneous Chemical Oscillators}

Typical chimera behavior, consisting of phase synchronized oscillators and phase drifting oscillators, was observed with group sizes of $N=40-50$ oscillators. With increasing group size, additional phase dispersed oscillators were found to coexist with the synchronized and asynchronous subgroups. The three coexisting subgroups were observed in populations of $\mathrm{N} \geq 60$. Figure 5.6 shows model simulation of the behavior of 90 identical BZ oscillators. Oscillators $j=62-82$ are phase sychronized with small phase shifts between neighboring oscillators. Within the syn- 
(a)

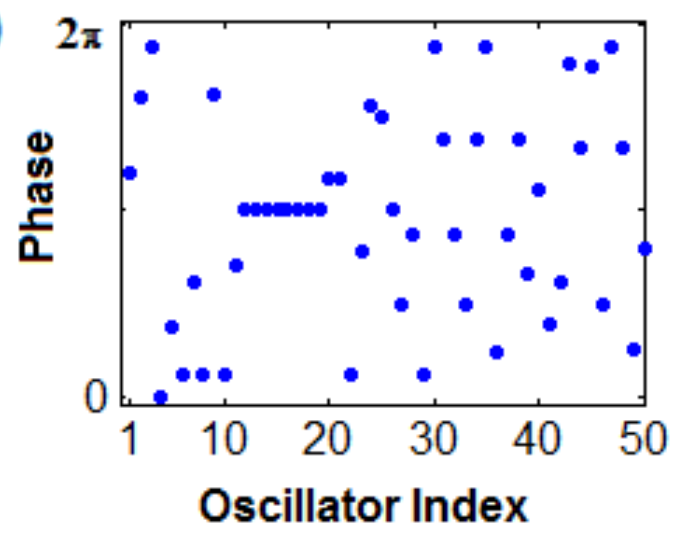

(c)

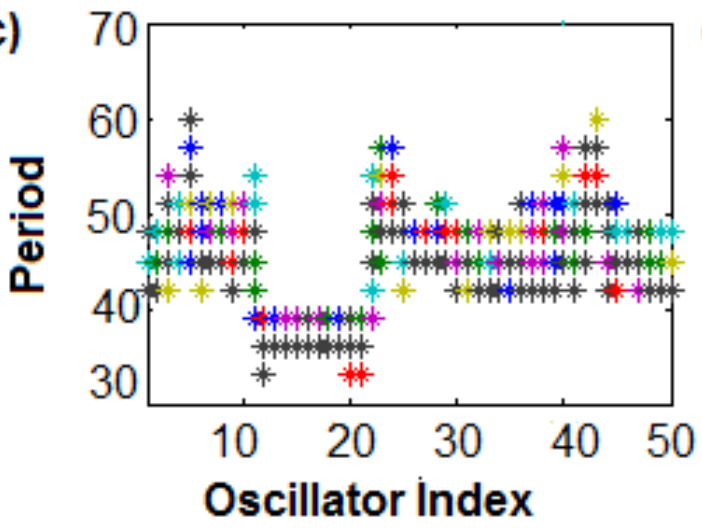

(b)

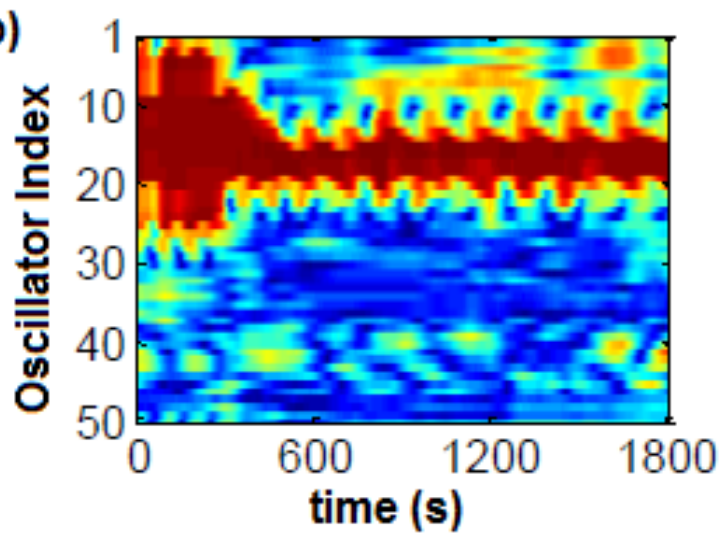

(d)

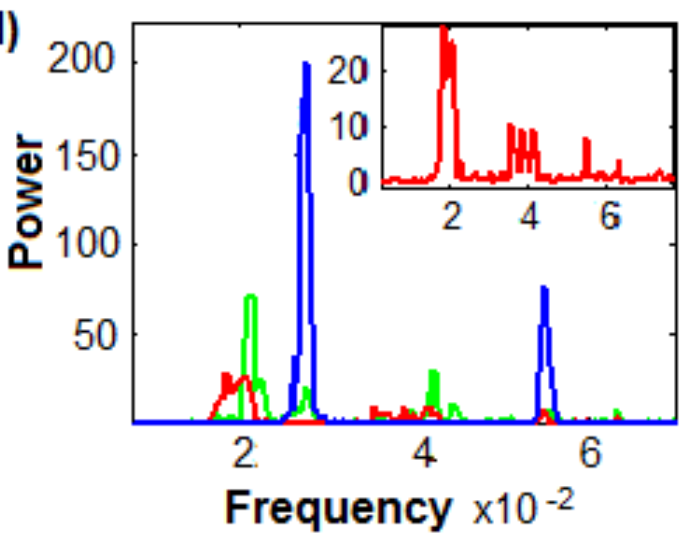

Figure 5.5. Experiment showing a chimera state. The experiment was started with a special initial phase distribution, in which some of the oscillators were synchronized. (a) A snapshot of the phase of each oscillator at $t=300 \mathrm{~s}$. (b) The local order parameter $\mathrm{R}$ as a function of time. (c) Period of each oscillator at 25 different times. (d) Power spectra of oscillators indexed $j=11$, 15, and 23. The insert shows an expanded view of oscillator $j=11$. 
chronized oscillators, the phase separation of oscillators increases with distance from the central oscillator. This gives the synchronized group a characteristic 'concave' shape, as shown in Figure $5.6(\mathrm{a})$.

Oscillators indexed $j=1-51$ and $j=84-90$ form the PD group, as shown in Figures 5.6(a) and 5.6(b). In Figure 5.6(b), we see that the oscillators have approximately the same period as their natural period of 41 . The asynchronous oscillators, $j=51-61$ and $j=76-83$, exist between the PD and synchronized groups. The asynchronous oscillators interact with the neighboring asynchronous oscillators, the PD oscillators, and the synchronized group, which all have different frequencies. As a result of the perturbations from these encounters, the oscillators exhibit a distribution of periods, as shown in Figure 5.6(b). The scattered points in the next return map of periods of oscillator $j=58$, shown in Figure 5.6(c), indicate aperiodic behavior. Oscillators closer to the synchronized group are sometimes entrained by the group. If the synchronized group drifts away, the oscillators closer to the PD group oscillate with their natural frequency. Plots of the period evolution of these oscillators have domains of periodic and aperiodic behavior. The behavior is summarized in Figure 5.6(d) for an interval of 1000 consecutive periods. The time series in Figure 5.6(e) shows the entrained neighboring oscillators $j=58$ (green) and $j=59$ (blue) drifting away from a synchronized group oscillator, $j=15$ (red).

The power spectra in Figure 5.7 shows a spread of frequencies ranging from the autonomous frequency through to the synchronized group frequency. The synchronized oscillators have a higher frequency than the other oscillators. The power spectra confirms the intermittent entrainment of oscillator $j=58$ by the synchronized group. 

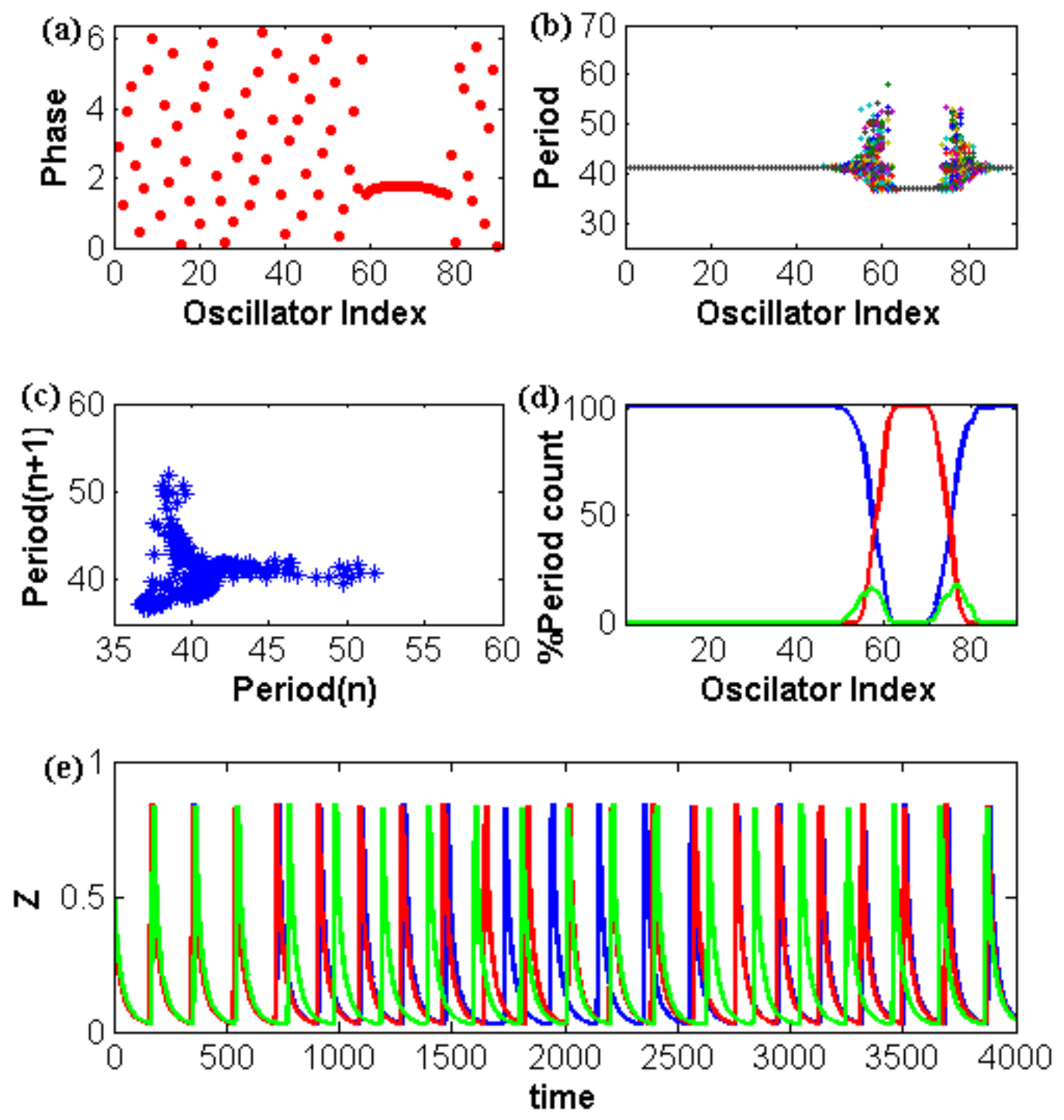

Figure 5.6. Model simulation with 90 homogeneous BZ oscillators. The simulation was started with a random initial phase distribution (a) A snapshot of the phase of each oscillator at time $2.9 \times 10^{5}$. (b) The corresponding scatter plot for 500 periods of each oscillator. (c) The next return period map of oscillator indexed $j=58$ exhibiting asynchronous behavior. (d) The percentage of the sampled 1000 periods each oscillator exhibits as synchronized (red), phase dispersed (blue), and aperiodic behavior (green). (e) Time series of 3 oscillators indexed $j=58$ (green), 59 (blue), and 75 (red). 


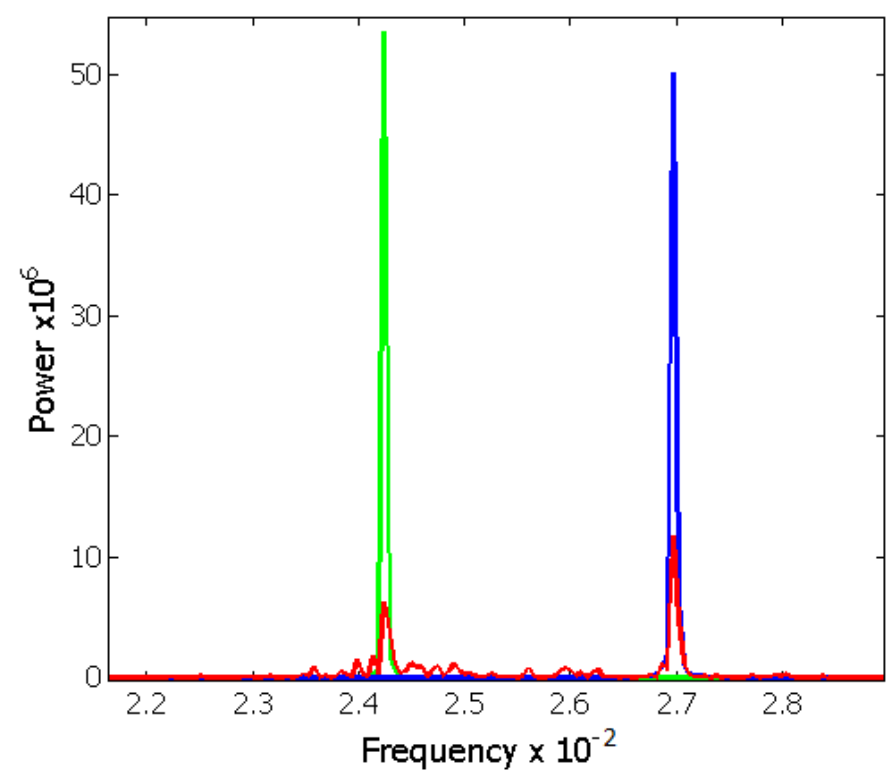

Figure 5.7. Power spectra of three oscillators; (a) $j=10$ from the phase dispersed group (green), (b) $j=58$ from the asynchronous group (red), and (c) $j=75$ from the synchronized group (blue).

\subsubsection{Chimera States in Heterogeneous Chemical Oscillators}

In Chapter 4, we studied chimera states in a group of 40 oscillators. Here, we analyze chimera state behavior in a larger group of 90 oscillators. The oscillators have a distribution in their natural period of $41 \pm 2.1$. In Figure 5.8(a), the snapshot of the phase of each oscillator at $t=9.0 \times 10^{4}$ shows a phase wave (PW) structure formed by oscillators $j=3-18$, a synchronized group $j=61-80$, and the asychronous oscillators. Phase waves were reported in experimental and simulation systems of heterogeneous oscillators [5] and have been discussed in Chapter 4. The period of the PW oscillators is less than the average natural period of the uncoupled oscillators; however, it is greater than that of the synchronized group, as shown in Figures 5.8(b) and (c). A scatter plot of 500 periods of each oscillator shows a distribution of periods for asynchronous oscillators and overlaying plots for the PW and synchronized oscillators. In the homogeneous system, the oscillators closer to the synchronized oscillators were sometimes entrained by the 

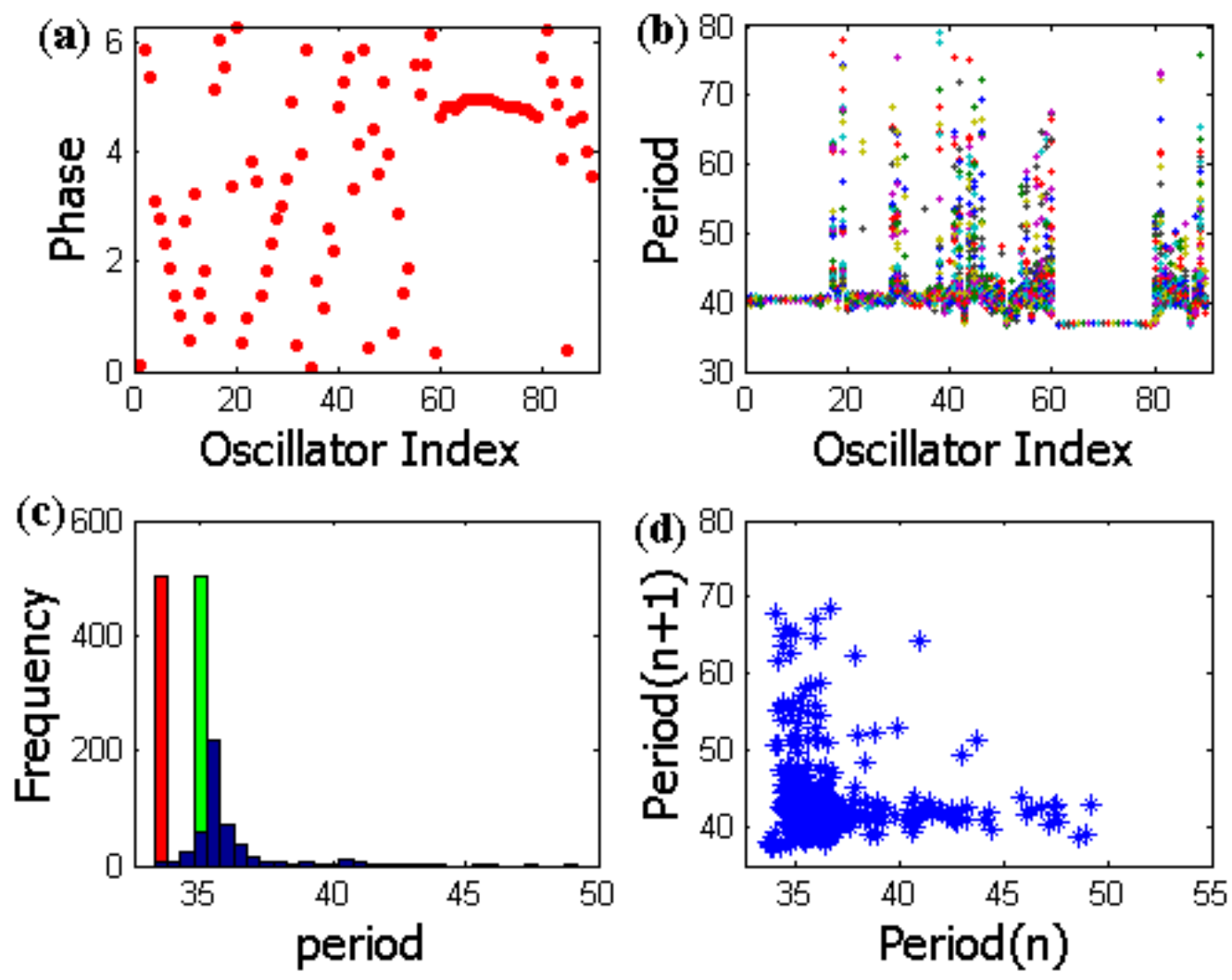

Figure 5.8. Model simulation with 90 heterogeneous BZ oscillators. The simulation was started with a random initial phase distribution (a) A snapshot of the phase of each oscillator at $\mathrm{t}=$ $9.0 \times 10^{4}$. (b) A scatter plot of 500 consecutive periods of each oscillator. (c) The distribution of 500 periods for oscillators exhibiting synchronized (red), phase wave (green), and aperiodic behavior (blue) indexed $j=10,45$, and 75, respectively. (d) The next return map of oscillator indexed $j=58$. 
group. In contrast, Figure 5.8(c) shows that the oscillators near the synchronized group are not always entrained by the synchronized group in heterogeneous systems. This was also observed in experiments, for example, oscillator $j=11$ in Figure 5.5(d). Although size fluctuations occur as in homogeneous systems, the synchronized behavior in heterogeneous systems does not meander as much as in identical oscillators. Figure 5.8(d) shows a next return map of the period evolution of oscillator $j=45$. The scattered points indicate aperiodic behavior of the oscillator. The aperiodic oscillators generally have broader distributions of periods in heterogeneous systems compared to the homogeneous systems. The time series of oscillators exhibiting different behaviors were analyzed using Fourier transforms and the power spectra are shown in Figure 5.9. As in the experiments and earlier simulations, the in-phase synchronized oscillators have a higher frequency compared to the synchronized phase-wave, as shown in Figure 5.9(a). The spectra also confirm the synchronized behavior within the phase wave. The power spectrum in Figure 5.9(b) shows a spread of frequencies in the region of the dorminant frequency of oscillator $j=43$. This is due to the aperiodic behavior of the oscillator.

\subsubsection{Phase-Cluster Chimera States}

Phase-cluster chimera states are composed of synchronized groups of oscillators separated by asynchronous group of oscillators. Classical chimera states have only one synchronized subgroup coexisting with an asynchronous subgroup. In simulations, phase-cluster chimera states were found with $0.3 \leq \kappa \leq 0.5$. The oscillators separating the synchronized groups interact with the out-ofphase clusters and asynchronous neighbors. Figure 5.10(a) shows a 2-phase-cluster chimera state with asynchronous oscillators indexed $j=9-13$ and $j=29-35$. The size of the clusters fluctuate with time, as the synchronized oscillators recruit neighbors or lose the end oscillators. In Figure $5.10(\mathrm{~b})$, the size of the incoherent group $(j=9-13)$ is reduced for $0 \leq \mathrm{t} \leq 60$, as the oscillators 

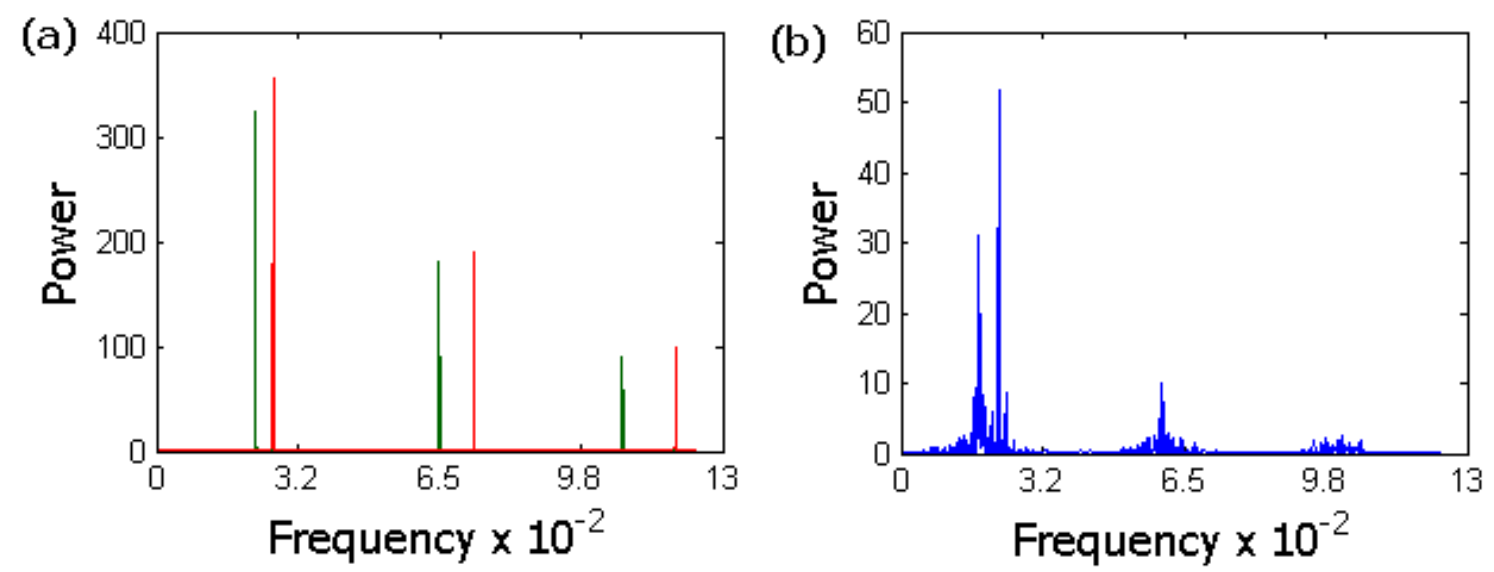

Figure 5.9. Power spectra of the Fourier transforms of the time series. (a) Spectra of phase wave oscillator indexed $j=10$ (red) and synchronized group oscillator $j=70$ (green). (b) Oscillator $j=43$ exhibiting asynchronous behavior.

are recruited by the synchronized groups. The asynchronous group $j=29-35$ then grows as more oscillators drift from the clusters. These asymmetric size fluctuations of the synchronized region cause irregular drifting of the synchronized behavior, as observed in Figure 5.10(d). The phase drifting oscillators have longer mean periods than the synchronized oscillators, as shown in Figure 5.10(c). For this reason, a plot of the mean period is capable of distinguishing the phase-cluster chimera from synchronized phase clusters, which have approximately the same period. The plot of the mean period as a function of oscillator index exhibits the expected chimera signature found in many studies. In Figure 5.10(d), the local order parameter shows the meandering and persistence of the behavior. 
(a)

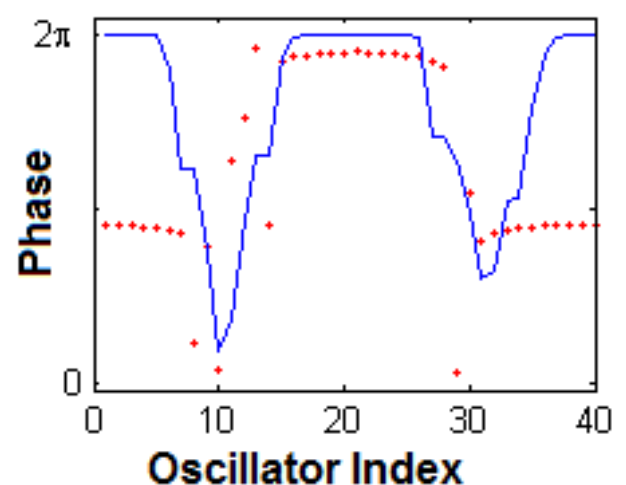

(c)

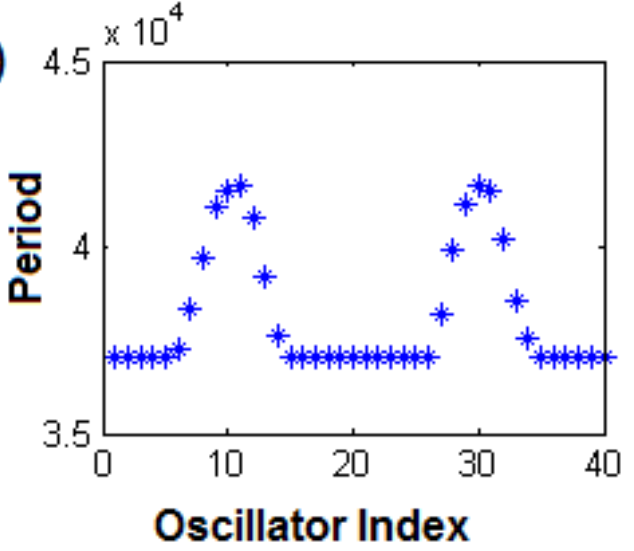

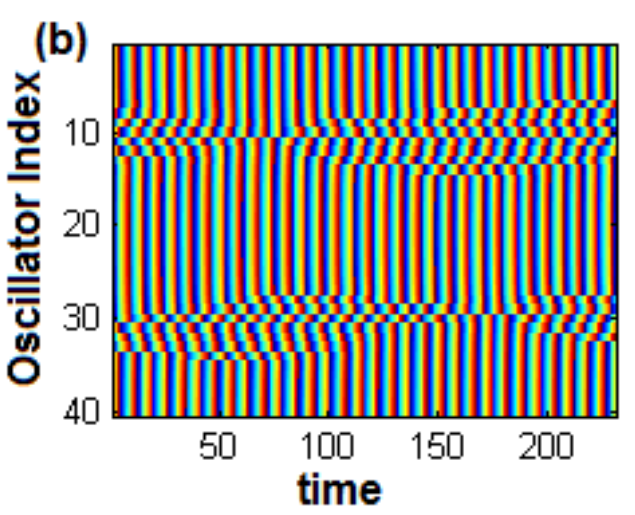

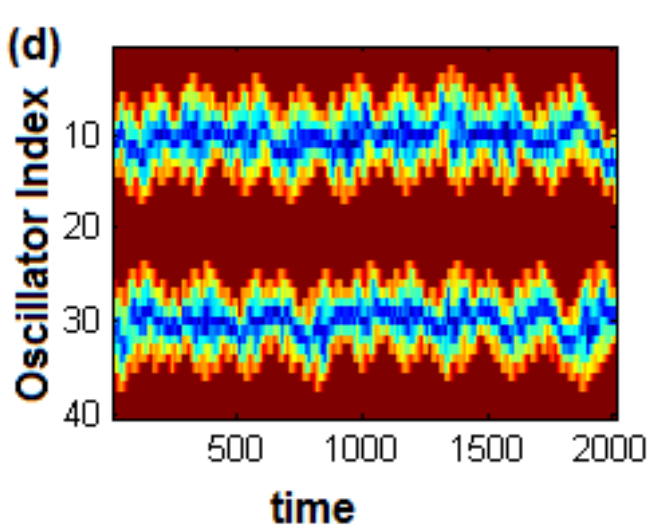

Figure 5.10. Phase-cluster chimera state observed in model simulations of 40 coupled heterogeneous BZ oscillators in a ring configuration, with $n=10, \kappa=0.4, K^{\prime}=6.3 \times 10^{-5}, \tau=35.0$, and $\phi_{0}=1.6 \times 10^{-4}$. The simulation was started with a random initial phase distribution. (a) Snapshot showing the phase of each oscillator at $t=1.80 \times 10^{2}$. (b) Phase of each oscillator as a function of time. (c) Mean period as function of oscillator index. (d) Local order parameter $R$ as a function of time, with $m=3$. 


\subsubsection{Periodic States}

\subsubsection{Phase Slip}

Phase slip behavior is characterized by oscillators periodically slipping from their clusters [18]. Figure 5.11(a) shows a snapshot of the slipping behavior of oscillators $j=15,16,35,36,55,56$, 75, and 76. In panel 5.11(b), we see all of the oscillators entrained by the synchronized clusters for $\mathrm{t}=0-30$. For $\mathrm{t}=30-75$, the oscillators go through the phase slipping cycle. The encounters of these slipping oscillators with the out-of-phase synchronized clusters cause period variations. The variations include phase advancement and delay. Phase delay results in longer periods and phase advancement in shorter periods. In Figure 5.11(c) (lower panel), we see that the oscillator is phase delayed as it slips from the group, which gives the first large peak. As it 'slips', it encounters the neighboring cluster, which causes a second phase delay. The magnitude of the second phase delay is generally smaller than the earlier delay response. When oscillator $j=15$ (lower panel) becomes phase delayed, the neghboring oscillator $j=14$ (upper panel) becomes phase advanced by a smaller magnitude. The oscillator is advanced more by the second pertubation, which phase advances oscillator $j=15$ toward the synchronized group. The period versus time plot shows a repeating pattern of an 11-period cycle. The oscillators involved in phase-slipping behavior have longer average periods, as is shown in Figure 5.11(d). In this example, the phase drifting oscillators do not spend a significant amount of time with the neighboring cluster during their 11-period cycle. In some examples, including in heterogeneous systems, the drifting oscillators are entrained by the neigboring cluster for at least 3 periods. Since these exhibit a temporary switch of clusters, we have categorized the phenomenon as switching behavior, which is discussed in the next section.

Phase slipping behavior occurs as an intermediate behavior between switchers and fully synchronized clusters. Phase slipping usually occurs in systems with a few drifting oscillators. In 
switchers and phase-cluster chimera states, the phase drifting oscillators have distributions in their periods and their average periods are longer compared to that of the synchronized oscillators. Using the example in Figure 5.11(c), we were able to demonstrate the periodicity of the phase slipping oscillators. The oscillators exhibit high periodicity and have longer mean periods similar to those of asynchronous oscillators in chimera states. The plots of the mean period of the phase-cluster chimera shown in Figure 5.10(c) and phase slipping system in Figure 5.11(d) show a similar chimera signature [1,2]; however, analysis of individual oscillator behavior revealed the coexistence of a high periodicity and synchronized groups of oscillators in the phase slipping system.

\subsubsection{Switching Behavior}

Switchers occur in systems with more than one cluster, where some oscillators switch between neighboring clusters. Switchers were reported in earlier studies of globally coupled populations of chemical oscillators $[19,20]$. We observed two types of switching behavior in populations of nonlocally coupled homogeneous and heterogeneous oscillators, periodic and aperiodic switching. Periodic switching is similar to phase slipping but typically involves drifting oscillators being entrained by the neighboring clusters for more than a period. Aperiodic switching is related to phase-cluster chimera states. In most examples, switching behavior is observed between out-ofphase clusters, as illustrated in Figure 5.12. The snapshot in Figure 5.12(a) shows phases of oscillators in a heterogeneous system exhibiting periodic switching behavior between clusters. The phase and local order parameter as a function of time are shown in Figures 5.12 (b) and 5.12(c), respectively. The switching oscillators are indexed $j=14$ and 38. Switching behavior was also observed in homogeneous systems.

Another type of periodic switching between an in-phase cluster and a phase wave was observed 

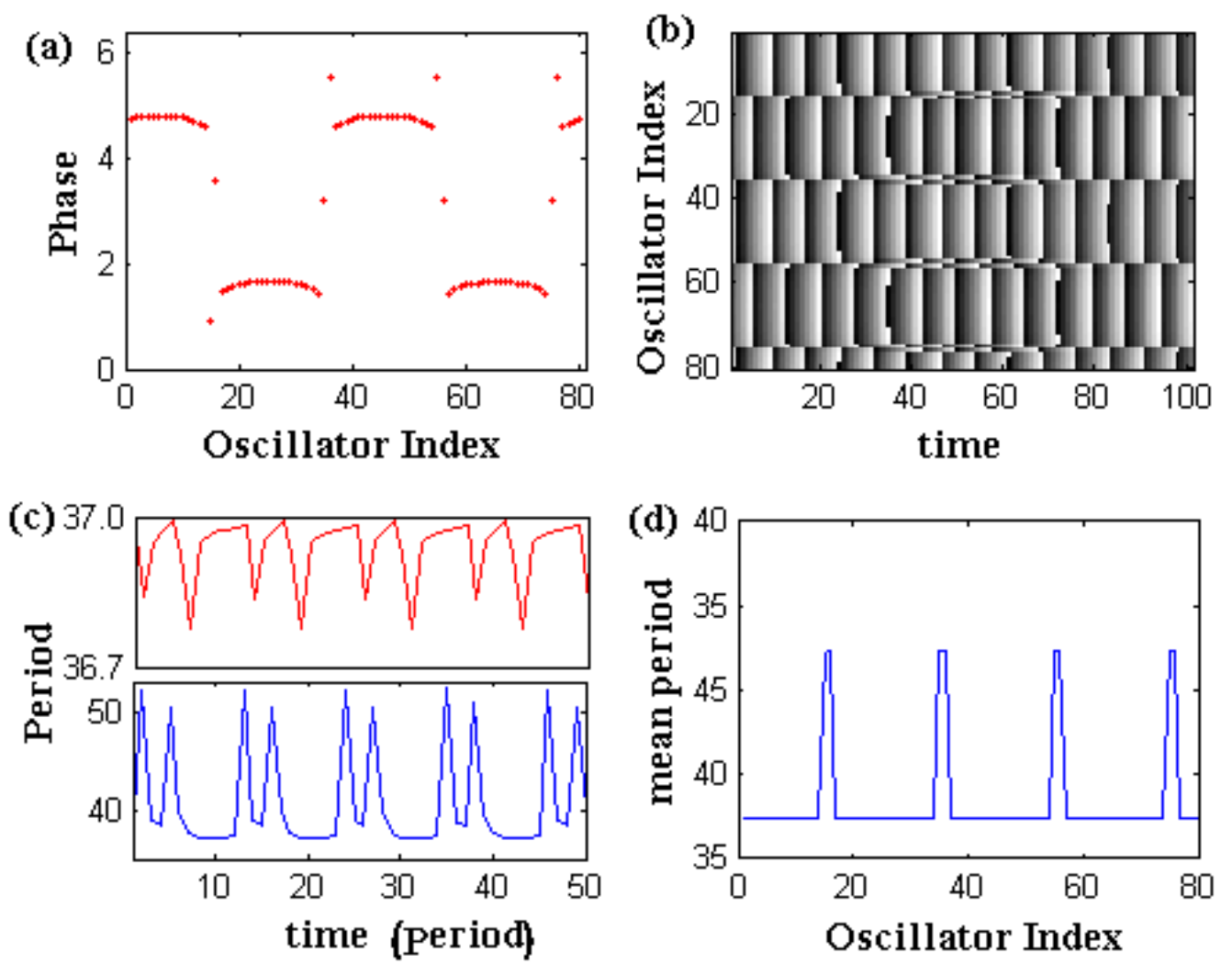

Figure 5.11. Simulation with 80 homogeneous BZ oscillators. (a) Snapshot of the phase of each oscillator showing phase-slip behavior at time $t=1.7 \times 10^{4}$. (b) Phase as a function of time of each oscillator. (c) The period evolution of the oscillators; $j=14$ (top panel) and $j=15$ (lower panel). (d) The average period as a function of time. 
(a)

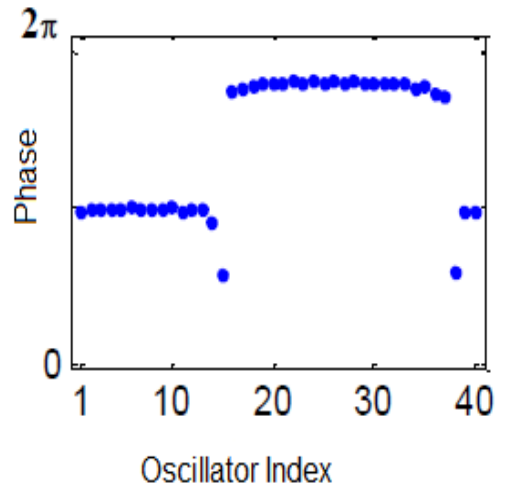

(b)

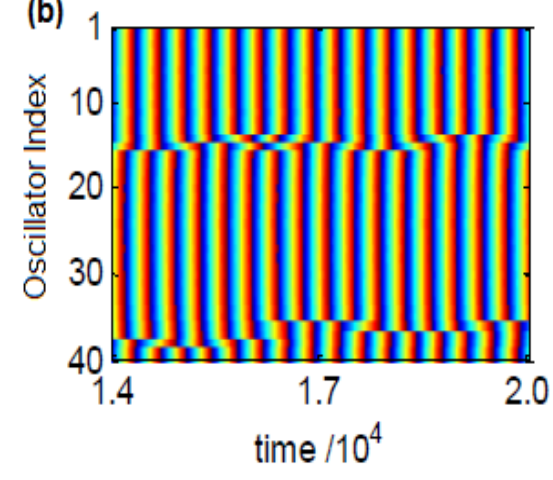

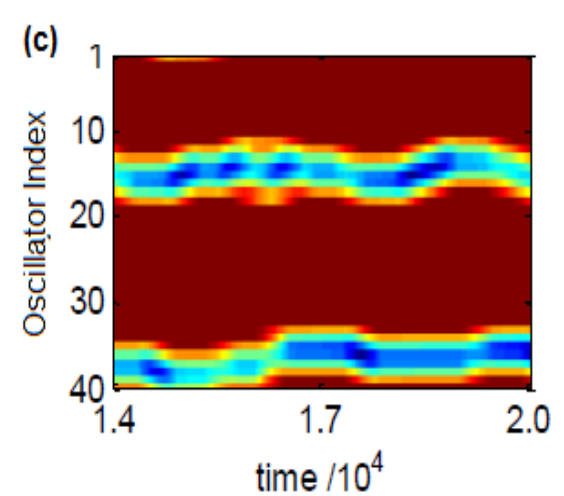

Figure 5.12. Simulation with 40 heterogeneous BZ oscillators. (a) Snapshot of the phase of each oscillator at time $t=1.9 \times 10^{4}$. (b) Phase of each oscillator as a function of time. (c) The local order parameter $\mathrm{R}$ as a function of time.

in simulations of 40 heterogeneous oscillators. Figure 5.13 shows a synchronized group next to a phase wave group of oscillators. In Figure 5.13(a), oscillators $j=1-16$ and $j=32-40$ form the synchronized group, while $j=18-23$ and $j=24-27$ form phase waves. Oscillators $j=17-18$ and $j=28-31$ show a distribution of periods due to encounters with neighbors with different frequencies. The histogram in Figure 5.13(b) is a summary of the distribution of 500 periods of oscillator $j=18$, which shows 3 subgroups, indicating the time the oscillator is entrained by the in-phase group, phase wave and switching between the groups. The insert in Figure 5.13(b) shows a time series of the period of oscillator $j=18$. The switching occurs over a 10 period cycle. The regular repeating pattern of period variations with time and the next return map in Figure 5.13(c) confirm the periodicity of the switching behavior. The behavior persisted for at least $10^{4}$ periods, suggesting that it is a stable behavior. Figure 5.13(d) shows a repeating pattern in the local order parameter for a length of time equivalent to $10^{3}$ oscillation periods. The oscillators $j=15-19$ and $j=28-33$ have local order parameter values that oscillate in time. Analysis of other systems with 
similar coexisting subgroups revealed that the periodic switching behavior is lost with increasing number of oscillators between the phase wave and the in-phase synchronized group, which was also demonstrated in Figure 5.8. This suggests that periodic behavior is more likely to occur in smaller group systems. The behavior discussed here has a chimera-like mean frequency signature although we have demonstrated periodicity of the switchers.

In the experiment shown in Figure 4.3, the periodic switching was not clear. This may be a result of the phase wave and synchronized groups not coexisting sufficiently long to see a repeating pattern. The phase waves often collapsed to a synchronized group over a long period of time in both simulations and experiments.

\subsubsection{Drifting Phase-Cluster Chimera State (DPCS)}

We carried out numerical investigations of the stability of the chimera states with 40 homogeneous oscillators by extending the simulations to 2000 oscillation periods. The simulation parameters used are $\kappa=0.4$ and $\rho=10$. Chimera states with one, two or three regions of coherent behavior (phase-clusters) were found. Local order parameter analysis of the 3-phase-cluster chimera revealed stable drifting of these coherent-incoherent patterns around the ring configuration. We investigated the occurrence and stability of the drifting phase-cluster chimera state by carrying out simulations for $10^{4}$ oscillation periods using special initial phase distributions. The results from 200 realizations are summarized in Figure 5.14. Only one realization had the chimera state collapsing to a fully synchronized state. The results show multistability of the n-phase cluster chimera states $(\mathrm{n}=1,2,3)$ and the fully synchronized state.

In most of the simulations, the classical chimera persisted for the duration of the simulation. A 2-cluster chimera state was observed in 58 realizations. The second and third clusters were oftenly formed through bifurcations of the existing synchronized group. Ocassionally a cluster was sponta- 

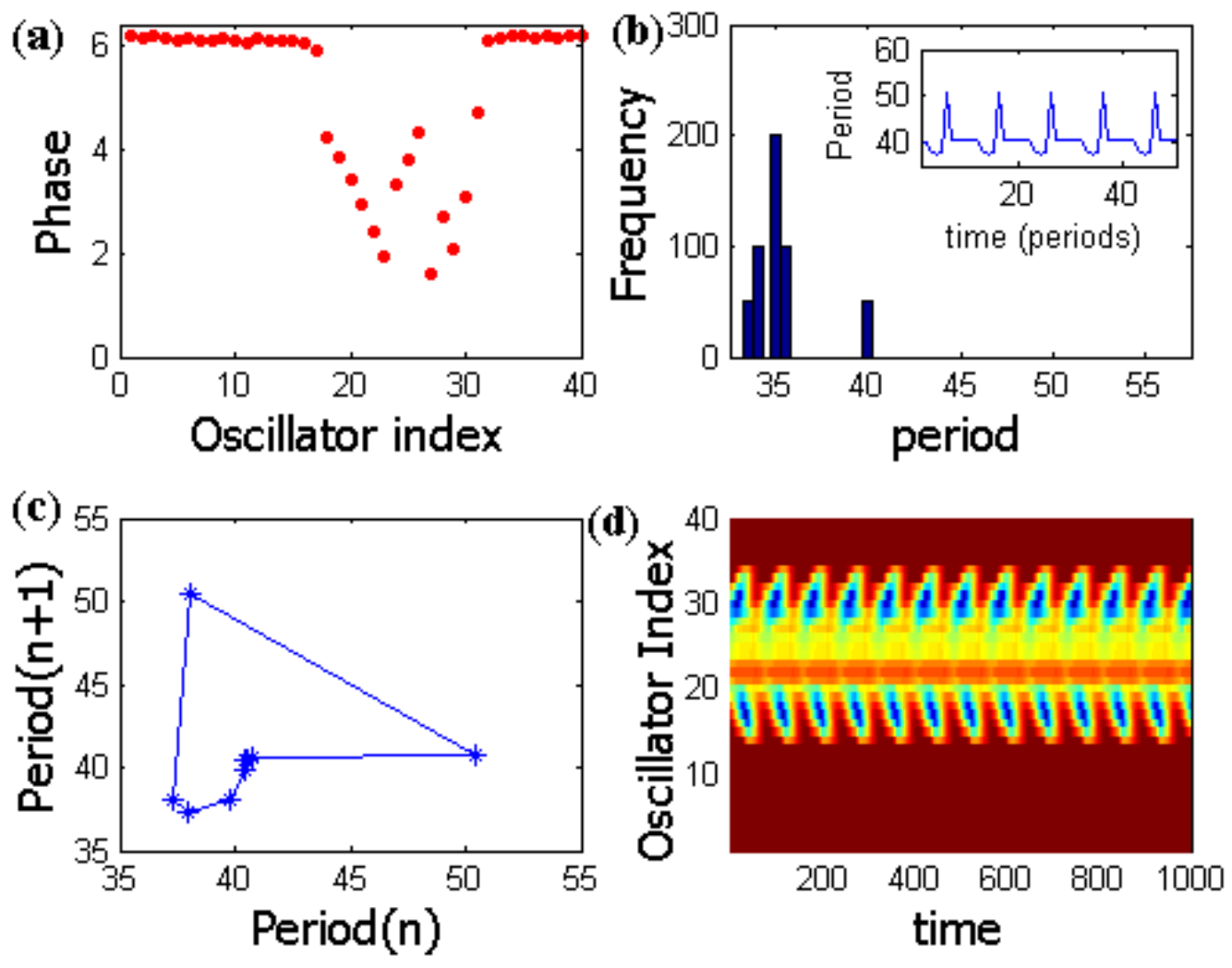

Figure 5.13. Model simulations of 40 coupled heterogeneous BZ oscillators in a ring configuration, with $n=10, \kappa=0.4, K^{\prime}=6.3 \times 10^{-5}, \tau=35.0$, and $\phi_{0}=1.6 \times 10^{-4}$. The simulation was started with a random initial phase distribution. (a) Snapshot showing the phase of each oscillator at $t=4.5 \times 10^{5}$. (b) The distribution of 500 periods of oscillator $j=18$. (c) Next return map of a periodically switching oscillator $j=18$. (d) Local order parameter $R$ as a function of time, with $m=3$. 


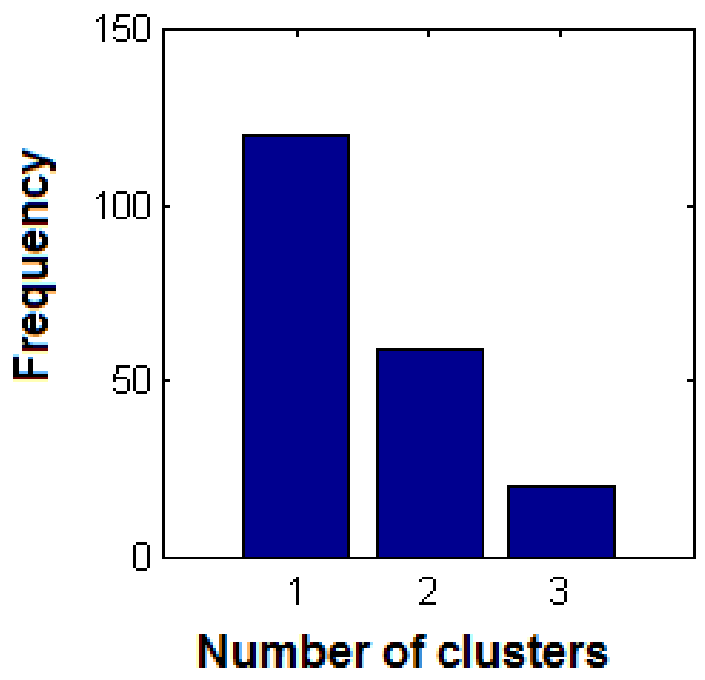

Figure 5.14. Distribution of phase-cluster chimera states from 200 realizations.

neously formed in a separate region. All 3-phase-cluster chimera states exhibited drifting patterns, which lasted for the duration of the experiment and drifted with an approximately constant linear velocity. Each oscillator exhibits time dependent periodic or aperiodic oscillations, which give rise to an oscillating local order parameter in time. Similar drifting patterns were observed in studies with the strongly coupled, nonlocal complex Ginzburg-Landau equation (NLCGLE) [14]. In [14], amplitude variations were considered due to strong coupling. In this system, we see the behavior with the same parameters as was used in examining other chimera behavior.

We studied the behavior for increasing $\mathrm{N}$ while keeping $\kappa$ and $\rho$ constant. DPCS were found in systems of up to 90 oscillators. Figure 5.15 shows a simulation initiated from a random phase distribution, which later exhibits DPCS behavior. A synchronized group of oscillators was spontaneously formed after 3000 time steps, and oscillators were recruited to this group. The cluster was made up of $13 \pm 2$ oscillators with small size fluctuations. Second, third, and fourth clusters were formed through bifurcations of one of the existing clusters, as shown in Figure 5.15(a). The time 


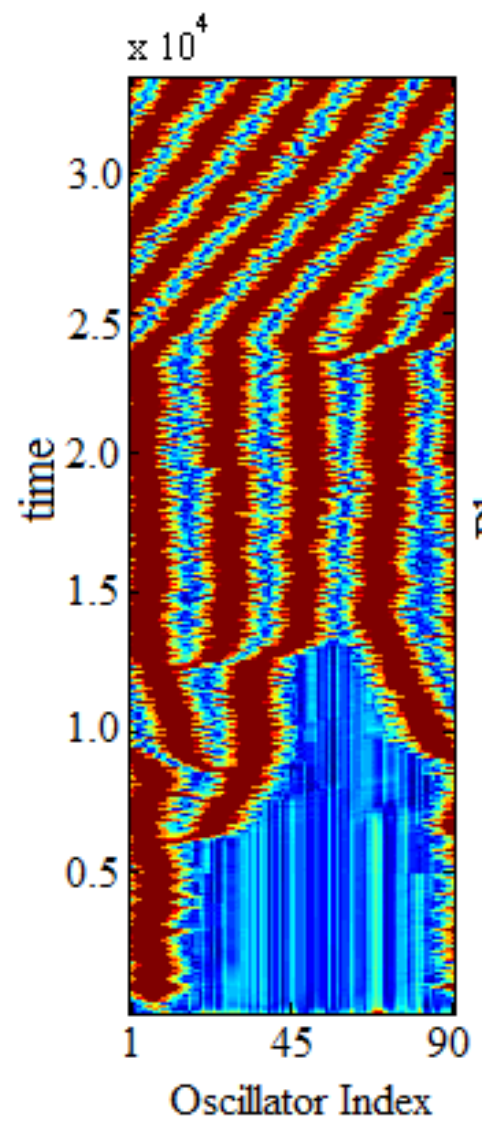

(a)
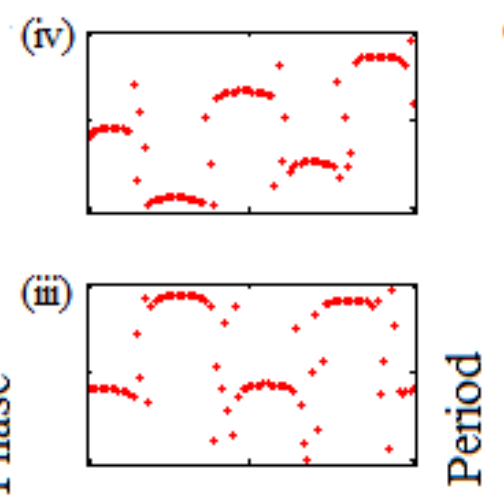

(ii)

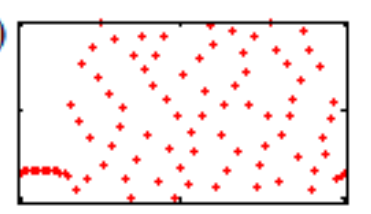

(i)

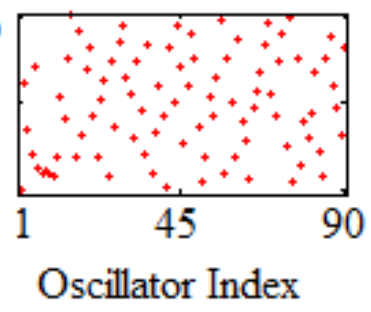

(b) (iv)

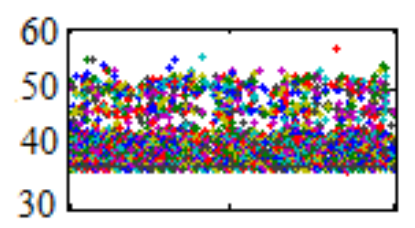

(iii)

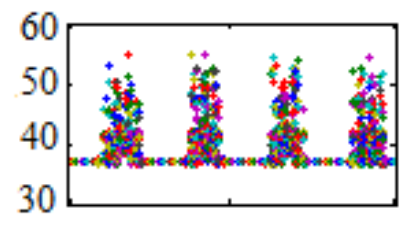

(ii)

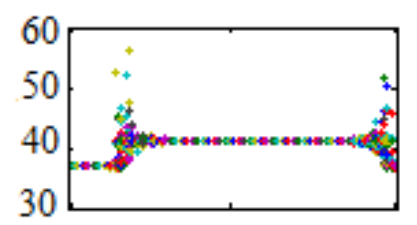

(i)

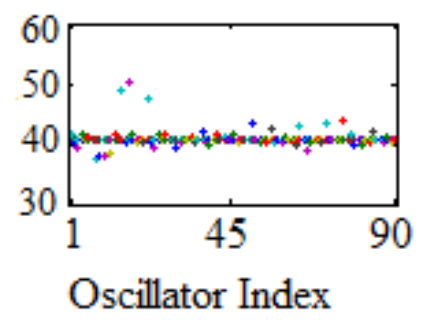

(c)

Figure 5.15. Model simulations of 90 coupled identical BZ oscillators. (a) Local order parameter $\mathrm{R}$ as a function of time. (b) Snapshot of the phase of each oscillator at four different times $t=3.0 \times 10^{3}, 4.0 \times 10^{3}, 2.0 \times 10^{4}$, and $3.2 \times 10^{4}$. (c) Scatter plot of each oscillator for 500 periods at four different time intervals. Simulations are initiated with a random initial phase distribution and were carried out for $10^{4}$ oscillation periods. 
interval between these new cluster formations varied from one bifurcation to the next and also from simulation to simulation. In this example, the four cluster system existed for an extended period of time before a bifurcation leading to formation of the fifth cluster occurred. The formation of the fifth cluster initiated the drift phenomenon. Figure 5.15(b)(i) - (iv) shows snapshots of the phase of each oscillator at four different times corresponding to the stages in Figure 5.15(a). Figure 5.15(c) shows scatter plots of 500 periods. The oscillators started with approximately the same period, as shown in Figure 5.15(c)(i). The synchronized group has a shorter period and the oscillators close to this group exhibit distributions of periods (see Figure 5.15(c)(ii)). The four clusters have approximately the same period and the oscillators between them show distributions in their periods. The clusters are arranged in pairs, which are $\pi$ out of phase with each other. Formation of the fifth cluster causes phase adjustments, and this initiates drifting of the coherent-incoherent patterns with evenly distributed clusters. In the DPCS, the oscillators have approximately the same mean period. The behavior was observed in populations of 40 - 90 oscillators, with number of clusters formed increasing with N. There was no evidence of drifting behavior in 4-cluster chimera states, which were formed with $N \geq 70$. DPCS with different occupancies and/or number of clusters can be observed with different values of $\kappa$ and $\rho$.

The variation of the linear velocity as a function of $\mathrm{N}$ is shown in Figure 5.16. The linear velocity was calculated in units of number of oscillators per period using the equation,

$$
\text { Linear velocity }=\frac{N \times T_{o s c}}{T_{d r i f t}}
$$

where $T_{\text {osc }}$ is the period of the synchronized group and $T_{\text {drift }}$ is the time it takes for a cluster to drift around the ring. The linear velocity decreases with $\mathrm{N}$, with more time required for the wave to make a complete cycle around the ring configuration with increasing $\mathrm{N}$. 


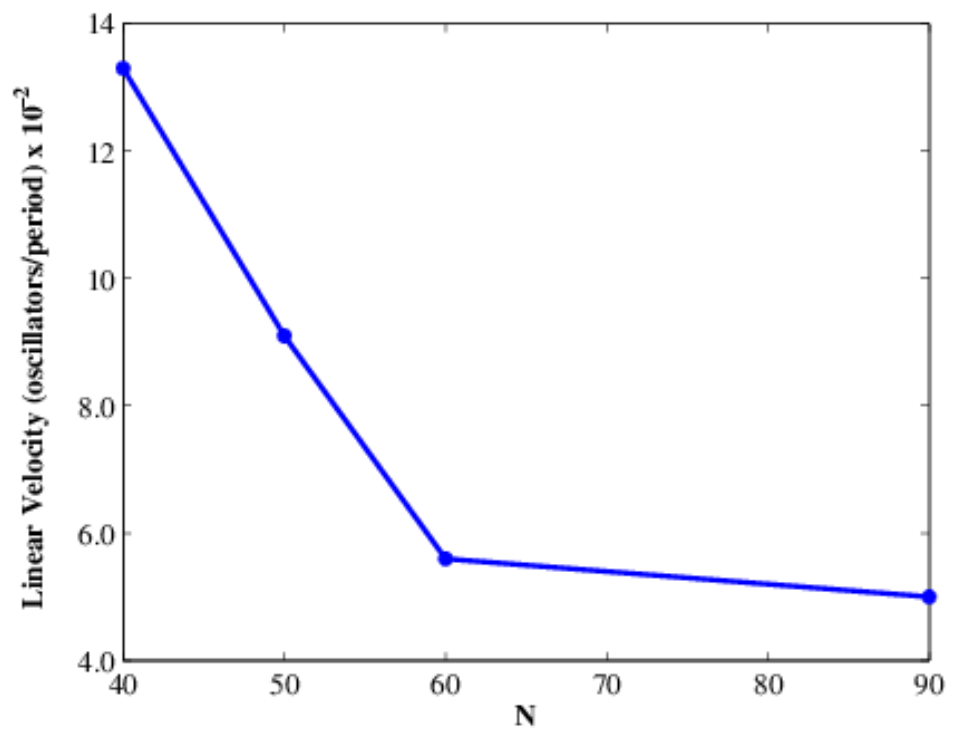

Figure 5.16. Linear velocity as a function of group size N.

\subsection{Summary}

Analysis of chimera states in both experiments and simulations confirmed the presence of coexisting subpopulations of oscillators exhibiting synchronous and asynchronous oscillations. From the discussion on homogeneous oscillators, we find that oscillators can phase lock in a phase dispersed state, thus making the phase distribution in a phase snapshot insufficient to characterize chimera behavior. Additional information about the aperiodic behavior of the oscillators was obtained from using scatter plots, next return maps, and Fourier transforms. Phase slipping and cluster switching behaviors have similar average period profiles as some phase-cluster chimera states in chemical oscillators [5] and phase oscillators [10], as well as multi-chimera states in FitzHugh-Nagumo (FHN) and Hindmarsh-Rose (HR) neuron models [8]. However, the period evolution as a function of time revealed that the switching oscillators exhibit periodic behavior, which could not be detected with plots of the mean period.

Early studies on chimera states emphasized the dependence of chimera behavior on the initial 
phase distribution $[17,21]$. With 40 homogeneous oscillators, with $\kappa=0.4$, the probability of finding a chimera state increased significantly when the system was started from special initial conditions compared to a random initial phase distribution. With heterogeneous oscillators, there was no significant difference in finding chimera states; however, using special initial conditions increased the likelihood of collapse to a fully synchronized state. This is consistent with the transient nature of chimera states reported in earlier studies $[6,22]$. The drifting phase-cluster chimera states existed for as long as the duration of the simulations ( $10^{4}$ periods).

In most phase-cluster chimera studies, the clusters have the same frequency. Recent studies by Yun et. al [23] revealed that clusters may have different frequencies in the presence of an external potential. Observations of states with more complex relations of coexisting subgroups will require new methods of characterizing these states. 


\section{References}

[1] Y. Kuramoto and D. Battogtokh, "Coexistence of coherence and incoherence in nonlocally coupled phase oscillators," Nonlinear Phenom. in Complex Syst. 5, 380-385 (2002).

Online Version 88, 91, 108

[2] D. M. Abrams and S. Strogatz, "Chimera states for coupled oscillators," Phys. Rev. Lett. 93, $174102(2004)$.

Online Version 88, 108

[3] D. M. Abrams, R. Mirollo, S. H. Strogatz, and D. A. Wiley, "Solvable model for chimera states of coupled oscillators," Phys. Rev. Lett. 101, 084103 (2008).

Online Version

[4] C. R. Laing, "Chimera states in heterogeneous networks." Chaos 19, 013113 (2009).

Online Version 88

[5] S. Nkomo, M. R. Tinsley, and K. Showalter, "Chimera states in populations of nonlocally coupled chemical oscillators," Phys. Rev. Lett. 110, 244102 (2013).

Online Version 88, 102, 116

[6] M. R. Tinsley, S. Nkomo, and K. Showalter, "Chimera and phase-cluster states in populations of coupled chemical oscillators," Nature Physics 8, 662-665 (2012).

Online Version 88, 117

[7] M. Wickramasinghe and I. Z. Kiss, "Spatially organized dynamical states in chemical oscillator networks: Synchronization, dynamical differentiation, and chimera patterns," PLoS ONE 8, e80586 (2013).

Online Version 88 
[8] I. Omelchenko, O. E. Omel'chenko, P. Hövel, and E. Schöll, "When nonlocal coupling between oscillators becomes stronger: patched synchrony or multichimera states," Physical Review Letters 110, 224101 (2013).

Online Version 88, 116

[9] J. Hizanidis, A. Kanas V.and Bezerianos, and T. Bountis, "Chimera states in networks of nonlocally coupled Hindmarsh-Rose neuron models," arXiv:1307.5452v1 [nlin.CD] (2013). 88

[10] G. C. Sethia, A. Sen, and F. M. Atay, "Clustered chimera states in delay-coupled oscillator systems." Phys. Rev. Lett. 100, 144102 (2008).

Online Version 88, 116

[11] R. Ma, J. Wang, and Z. Liu, "Robust features of chimera states and the implementation of alternating chimera states," Europhys. Lett. 91, 40006 (2010).

Online Version

[12] J. H. Sheeba, V. K. Chandrasekar, and M. Lakshmanan, "Globally clustered chimera states in delay-coupled populations." Phys. Rev. E 79, 055203 (2009).

Online Version

[13] J. H. Sheeba, V. K. Chandrasekar, and M. Lakshmanan, "Chimera and globally clustered chimera: Impact of time delay." Phys. Rev. E 81, 046203 (2010).

Online Version 88

[14] G. C. Sethia, A. Sen, and G. L. Johnston, "Amplitude-mediated chimera states," Phys. Rev. E 88, 042917 (2013).

Online Version 88, 113 
[15] S. R. Ujjwal and R. Ramaswamy, "Chimeras with multiple coherent regions," Phys. Rev. E 88, $032902(2013)$.

Online Version 88

[16] O. E. Omel'chenko, "Coherence-incoherence patterns in a ring of non-locally coupled phase oscillators," Nonlinearity 26, 2469-2498 (2013).

Online Version 88

[17] D. M. Abrams and S. H. Strogatz, "Chimera states in a ring of nonlocally coupled oscillators," Int. J. Bifurcation Chaos 16, 21-37 (2005).

Online Version 91, 117

[18] Z. Zheng, G. Hu, and B. Hu, "Phase slips and phase synchronization of coupled oscillators," Phys. Rev. Lett. 81, 3-6 (1998).

Online Version 107

[19] A. F. Taylor, P. Kapetanopoulos, B. J. Whitaker, R. Toth, L. Bull, and M. R. Tinsley, "Clusters and switchers in globally coupled photochemical oscillators." Phys. Rev. Lett. 100, 214101 (2008).

Online Version 108

[20] A. F. Taylor, M. R. Tinsley, F. Wang, and K. Showalter, "Phase clusters in large populations of chemical oscillators," Angew. Chem. Int. Ed. 50, 10161-10164 (2011). Online Version 108

[21] O. E. Omel'chenko, M. Wolfrum, S. Yanchuk, Y. L. Maistrenko, and O. Sudakov, "Stationary patterns of coherence and incoherence in two-dimensional arrays of non-locally-coupled phase 
oscillators," Phys. Rev. E 85, 036210 (2012).

Online Version 117

[22] M. Wolfrum and O. E. Omel'chenko, "Chimera states are chaotic transients." Phys. Rev. E 84, 015201 (2011).

Online Version 117

[23] Z. Yun, Z. Zhi-Gang, and Y. Jun-Zhong, "Four-cluster chimera state in non-locally coupled phase oscillator systems with an external potential," Chinese Physics B 22, 100505 (2013). Online Version 117 


\title{
Synchronization Behavior in Coupled Chemical Oscillators
}

\author{
Simbarashe Nkomo \\ Dissertation submitted to the \\ Eberly College of Arts and Sciences \\ at West Virginia University \\ in Partial Fulfillment of the Requirements for the Degree of \\ Doctor of Philosophy \\ in
}

Chemistry

APPROVAL OF THE EXAMINATION COMMITTEE

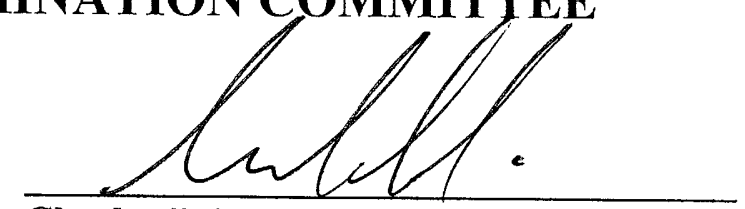

Charles Jaffe, Ph.D.

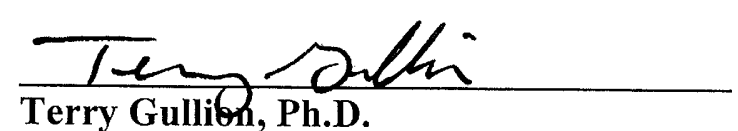

Terry Gullion, Ph.D.

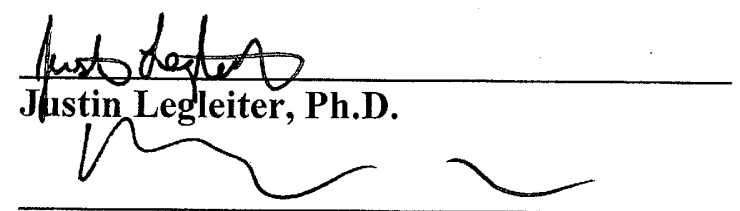

Mark R. Tinsley, Ph.D.

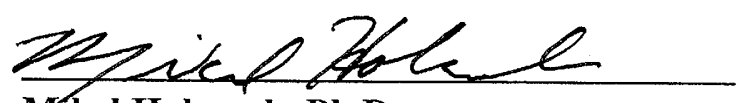

Míkel Holcomb, Ph.D.

$\frac{4 / 10 / 2014}{\text { Date }}$

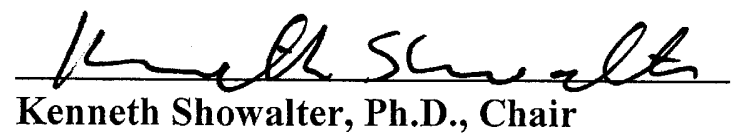

